Pacific Journal of Mathematics

RESIDUE CLASS DOMAINS OF THE RING OF CONVERGENT 


\title{
RESIDUE CLASS DOMAINS OF THE RING OF CONVERGENT SEQUENCES AND OF $C^{\infty}([0,1], \mathbf{R})$
}

\section{JAMES J. MOLONEY}

\begin{abstract}
We show that there are exactly 10 residue class domains of $c$, the ring of real convergent sequences. We also classify some of the residue class domains of $C^{\infty}([0,1], \mathbf{R})$.
\end{abstract}

Introduction. The residue class domains of $C(X, \mathbf{R})$ (the ring of real valued continuous functions on a topological space $X$ ) have been extensively studied by Kohls [16], Gillman and Jerison [10], and others.

In [6], Cherlin and Dickmann began the study of the residue class domains of $C\left(\mathbf{N}^{*}, \mathbf{R}\right)$, where $\mathbf{N}^{*}$ is the one-point compactification of $\mathbf{N}$. Clearly $C\left(\mathbf{N}^{*}, \mathbf{R}\right)$ is isomorphic to $c$, the ring of real convergent sequences. In [8], Cherlin and Dickmann (and Louveau) showed that there exist non-maximal prime ideals $p$ of $C\left(\mathbf{N}^{*}, \mathbf{R}\right)$ such that $C\left(\mathbf{N}^{*}, \mathbf{R}\right) / p$ is a real closed valuation ring. They asked about the other prime ideals. Both the author's dissertation (written under Cherlin's guidance) and this paper grew out of that question. We deal with two main problems:

(1) Classify all the residue class domains of $C\left(\mathbf{N}^{*}, \mathbf{R}\right)$, assuming the continuum hypothesis.

(2) Classify the residue class domains of $C^{\infty}([0,1], \mathbf{R})$.

(The reader may well ask, "What about $C([0,1], \mathbf{R})$ ?" We touch this question very lightly. Cherlin and Dickmann, in $\S 4$ of [8], go into it more deeply.)

We completely solve problem 1 in this paper. We show that there are exactly ten residue class domains of $C\left(\mathbf{N}^{*}, \mathbf{R}\right)$.

We can classify these domains in the following manner, considering $c$ rather than $C\left(\mathbf{N}^{*}, \mathbf{R}\right)$ :

First, one of these conditions will hold for $\left(l^{\infty} / p-c / p\right)$; it will either be empty, have a non-empty countable coinitial subset, or be non-empty with no countable coinitial subset.

Second, one of these conditions will hold on neighborhoods of zero in $c / p$; there will either be a countable coinitial subset of $c / p$ of the form $\left\{[f]^{k}\right\}_{k=1}^{\infty}$, or a countable coinitial subset $\left\{\left[f_{m}\right]\right\}_{m=1}^{\infty}$ of $c / p$ 
where $\left[f_{m}\right]^{k}>\left[f_{m+1}\right]$ for all $k, m \in \mathbf{N}$, or there will be no countable coinitial subset of $c / p$.

This accounts for nine of the domains; $\mathbf{R}=c / \mathbf{m}$, where $\mathbf{m}$ is any maximal ideal of $c$, is the tenth.

Our main line of attack is to use some work of Ax and Kochen [1] concerning valued fields. (The use of valuations to study $C(X, \mathbf{R}) / p$ goes back at least to [16].) In [14] it was shown that, under certain conditions, two valued fields will be isomorphic if and only if both the two valuation groups and the two residue class fields are isomorphic. For a non-maximal prime ideal $p$ of $c$, we have a chain of at most three fields:

$$
\mathbf{R} \subset \operatorname{Frac}\left(l^{\infty} / c_{0}\right) \subset \operatorname{Frac}(c / p)
$$

(where $c_{0}$ is the set of sequences that converge to zero, and $\operatorname{Frac}(D)$ denotes the fraction field of a domain $D$ ). The question then comes down to: "What are the possible valuation groups of $\operatorname{Frac}\left(l^{\infty} / c_{0}\right)$ over $\mathbf{R}$, and what are the possible valuation groups of $\operatorname{Frac}(c / p)$ over $\operatorname{Frac}\left(l^{\infty} / c_{0}\right)$ ?" We shall show that there are three in each case.

In the first case, $\operatorname{Frac}\left(l^{\infty} / c_{0}\right)$ over $\mathbf{R}$, the valuation group is closely tied to $\left(l^{\infty} / p-c / p\right)$. If $l^{\infty} / p=c / p$, then the valuation group is the group $\{0\}$. If $l^{\infty} / p \neq c / p$, then $\left(l^{\infty} / p-c / p\right)_{+}$having a countable coinitial subset is equivalent to the valuation group having a countable cofinal subset. These conditions are closely tied to different types of non-principal ultrafilters on $\mathbf{N}$.

In the second case, $\operatorname{Frac}(c / p)$ over $\operatorname{Frac}\left(l^{\infty} / p\right)$, the valuation group is closely tied to the neighborhoods of zero in $c / p$. These neighborhoods of zero will have a countable coinitial subset if and only if the valuation group has a countable cofinal subset. Further, these neighborhoods of zero will have a coinitial subset of the form $\left\{[f]^{k}\right\}_{k=1}^{\infty}$, if and only if the valuation group has a cofinal subgroup isomorphic to $\mathbf{R}$.

Non-principal ultrafilters on $\mathbf{N}$ play an extremely large role in this paper, since every non-maximal prime ideal $p$ of $c$ has such an ultrafilter associated with it. For a very good survey article on these non-principal ultrafilters, see [23].

Problem 2 is considerably more complicated. There are several reasons for this. Among them are the exceedingly richer prime ideal structure of $C^{\infty}([0,1], \mathbf{R})$ and the fact that not all convergent sequences can be extended to $C^{\infty}$ functions.

We are able to solve a small piece of this problem, by using the results of problem 1 . Certain quotient rings of $C^{\infty}([0,1], \mathbf{R})$ are iso- 
morphic to subrings of $c$. For some of these, we completely classify the "inherited" residue class domains. We also classify the residue class domains associated with the largest differentially closed subideals of these inherited ideals. These latter domains are differential rings, and have themselves a very complicated structure.

Finally, we show the existence of a prime ideal which is neither inherited from $C([0,1], \mathbf{R})$ nor a differentially closed sub-ideal of an inherited ideal. In fact, we show the existence of a prime ideal $p$ of $C^{\infty}([0,1], C)$ which is not equal to its own complex conjugate.

In $\S 1$ we review certain preliminaries. In $\S 2$, we show that there are at most 10 residue class domains of $c$, depending on whether or not all three cases of coinitial subset of $\left(l^{\infty} / p-c / p\right)_{+}$, mentioned earlier, can occur. In $\S 3$, we show that all three cases do, in fact, occur. In $\S 4$, we apply these results to some other spaces. In $\S 5$, we study the residue class domains of $C^{\infty}([0,1], \mathbf{R})$.

I would like to thank my thesis advisor, Professor Gregory Cherlin, who suggested this problem and the main lines of attack to me, while I was his Ph.D. student at Rutgers University.

\section{Preliminaries.}

A. Real closed fields. Here are some well known facts concerning real closed fields, which will bring us to the Ax-Kochen Theorem, which plays a significant role in this approach to classifying the residue class domains of $c$.

Definition 1.1. A real closed field is an ordered field such that:

(i) Every positive element of $F$ has a square root in $F$.

(ii) Every odd degree polynomial, $P \in F[x]$, has a zero in $F$.

We denote by $C(X, \mathbf{R})$ the ring of all real valued continuous functions defined on a topological space $X$.

(1.2) If $\mathbf{m}$ is a maximal ideal of $C(X, \mathbf{R})$, then $C(X, \mathbf{R}) / \mathbf{m}$ is a real closed field ([10], p. 175).

Considering non-maximal prime ideals of $C(X, \mathbf{R})$, we will find the following to be useful.

Proposition 1.3. Let $p$ be a prime ideal of $C(X, \mathbf{R})$. Then, $\operatorname{Frac}(C(X, \mathbf{R}) / p)$ is a real closed field.

Proof. $C(X, \mathbf{R}) / p$ is ordered by ([10], p. 69). In proving (1.2), Gillman and Jerison showed that in $C(X, \mathbf{R}) / p$, every polynomial of 
odd degree with leading coefficient 1 has zero in $C(X, \mathbf{R}) / p$. Trivially, every positive element has a square root.

Definition 1.4. A real closed ring $A$ is an ordered domain such that

(i) Every positive element of $A$ has a square root in $A$.

(ii) Given any polynomial $P \in A[x]$ and $a, b \in A, a<b$, if $P$ changes sign from $a$ to $b$, then $P$ has a zero $c$ such that $a<c<b$.

We use $\mathbf{N}^{*}$ to denote the one-point compactification of the natural numbers. Cherlin and Dickmann (and Louveau) have shown that:

(1.5) There exist non-maximal prime ideals of $C\left(\mathbf{N}^{*}, \mathbf{R}\right)$ such that $C\left(\mathbf{N}^{*}, \mathbf{R}\right) / p$ is a real closed ring $([8], 3.2 .2)$.

We are interested in the other quotient domains as well. The following is well known:

(1.6) The following are equivalent:

(i) $A$ is a real closed ring.

(ii) $A$ is a convex subring of a real closed field and $l \in A$.

B. Filters. Every prime ideal has associated with it a prime $z$-filter. For $X=\mathbf{N}^{*}$ every non-maximal prime $z$-filter has a corresponding non-principal ultrafilter on $\mathbf{N}$. These facts we shall use extensively throughout this paper.

Here we recall some simple properties of filters which will be assumed in later arguments.

The zero set, $f^{-1}(\{0\})$, of a function $f \in C(X, \mathbf{R})$ is denoted by $Z(f)$.

Definition 1.7. A $z$-filter is a family $\mathscr{F}$ of zero sets such that

(i) $Z(f) \in \mathscr{F}$ and $Z(g) \in \mathscr{F}$ imply $[Z(f) \cap Z(g)] \in \mathscr{F}$.

(ii) $Z(f) \in \mathscr{F}, Z(f) \subset Z(g)$ imply $Z(g) \in \mathscr{F}$.

The filter $\mathscr{F}$ is proper if

(iii) $\phi \notin \mathscr{F}$.

DEFINITION 1.8. A $z$-filter is prime if $[Z(f) \cup Z(g)] \in \mathscr{F}$ implies $Z(f) \in \mathscr{F}$ or $Z(g) \in \mathscr{F}$.

Prime $z$-filters are crucial to the study of residue class domains. In particular, we shall find useful the following theorems.

(1.9) Given a topological space $X$ and a prime $z$-filter $\mathscr{F}$ on $X$, the set of functions

$$
p_{\mathscr{F}}=\{f \mid Z(f) \in \mathscr{F}\}
$$

is a prime ideal ([10], p. 29). 
(1.10) Given a prime ideal $p$ of $C(X, \mathbf{R})$, there exists a prime $z$ filter $\mathscr{F}$ such that $Z(f) \in \mathscr{F}$ implies $f \in p$. That is, $p_{\mathscr{F}} \subset p([\mathbf{1 0}]$, p. 197).

Definition 1.11. An ultrafilter on a set $S$ is a family $\mathscr{U}$ of subsets of $S$, such that

(i) $A \in \mathscr{U}$ and $B \in \mathscr{U}$ imply $(A \cap B) \in \mathscr{U}$.

(ii) $A \in \mathscr{U}$ and $A \subset B$ imply $B \in \mathscr{U}$.

(iii) $\phi \notin \mathscr{U}$.

(iv) For all $A \subset S$, either $A \in \mathscr{U}$ or $(S-A) \in \mathscr{U}$.

Notation 1.12 . For an ultrafilter $\mathscr{U}$ on a set $X$, and for functions $f, g: X \rightarrow \mathbf{R}$

$$
\begin{array}{lll}
f \underset{\overline{\mathscr{U}}}{\equiv} g & \text { means } & \{x \mid f(x)=g(x)\} \in \mathscr{U} . \\
f_{\mathscr{U}}^{>} g & \text { means } & \{x \mid f(x)>g(x)\} \in \mathscr{U} .
\end{array}
$$

For a prime $z$-filter $\mathscr{F}$ on a topological space $X$, and for $f, g \in$ $C(X, \mathbf{R})$

$$
\begin{gathered}
f \underset{\mathscr{F}}{\equiv} g \text { means }\{x \mid f(x)=g(x)\} \in \mathscr{F} . \\
f \underset{\mathscr{F}}{g} \text { means }\{x \mid f(x) \geq g(x)\} \in \mathscr{F} \text { but } f \underset{\mathscr{F}}{\not \equiv} g .
\end{gathered}
$$

We will need the following theorems on ultrafilters.

(1.13) Let $\mathscr{F}$ be a non-maximal prime $z$-filter on $\mathbf{N}^{*}$. Then there exists an ultrafilter $\mathscr{U}(\mathscr{F})$ on $\mathbf{N}$ such that for $A \subset \mathbf{N}$

$$
A \in \mathscr{U}(\mathscr{F}) \leftrightarrow(A \cup\{\infty\}) \in \mathscr{F}
$$

([17], Th. 2.2).

REMARK 1.14. For $\mathscr{F}$ and $\mathscr{U}(\mathscr{F})$ as above, letting $c$ denote the ring of convergent real sequences and letting

$$
p_{\mathscr{U}(\mathscr{F})}=\{f \in c \mid Z(f) \in \mathscr{U}(\mathscr{F})\},
$$

we have

$$
c / p_{\mathscr{U}(\mathscr{F})} \simeq C\left(\mathbf{N}^{*}, \mathbf{R}\right) / p_{\mathscr{F}} .
$$

(1.15) $C(X, \mathbf{R}) / p$ is always an ordered domain ([10], p. 69).

(1.16) For a prime ideal $p$ of $C(X, \mathbf{R})$, if $\mathscr{F}$ is the prime $z$-filter such that

$$
Z(f) \in \mathscr{F} \quad \text { implies } f \in p
$$


then

([16], p. 69).

$$
\{x \mid f(x) \geq g(x)\} \in \mathscr{F} \quad \text { implies } \quad[f] \geq[g]
$$

Combining (1.14) and (1.16) we have

(1.17) Let $p$ be a prime ideal of $c$. Let $\mathscr{U}$ be the ultrafilter such that

$$
\{n \mid f(n)=0\} \in \mathscr{U} \quad \text { implies } f \in p .
$$

Then

$$
f \underset{\mathscr{U}}{>} g \text { implies }[f] \geq[g] \text {. }
$$

Definition 1.18. For $\mathscr{F}$ and $p$ as in (1.10), we call $\mathscr{F}$ the prime $z$-filter associated with $p$. For $\mathscr{U}$ and $p$ as in (1.17), we call $\mathscr{U}$ the ultrafilter associated with $p$.

Notation 1.19. $c_{0}$ denotes the set of real valued sequences converging to zero. $l^{\infty}$ denotes the ring of bounded real valued sequences.

REMARK 1.20. Let $p$ be a non-maximal prime ideal of $c$. Then $p$ is properly contained in $c_{0}$.

As a result of the comparison test, we have

REMARK 1.21. $c_{0}$ is a convex subset of $l^{\infty}$. In fact $c_{0}$ is a convex ideal of $l^{\infty}$.

C. Valuation theory. Back in [16], Kohls began using valuation theory to study residue class domains of $C(X, \mathbf{R})$. Since that time, valuation theory has been greatly developed by Ax and Kochen. We will use their results to classify the residue class domains of $c$, giving the fraction field of $c / p$ ( $p$ being a non-maximal prime ideal of $c$ ) the structure of a valued field.

Definition 1.22. A valued field is a 6-tuple $(K, R, I, G, v, \bar{K})$, where

(i) $K$ is the field, hereafter called the main field.

(ii) $R$ is a subring of $K$ such that for all $a \in K$, either $a \in R$ or $a^{-1} \in R$.

$R$ is called the valuation ring.

(iii) $I=\left\{a \in R \mid a^{-1} \notin R\right\}$.

$I$ is called the valuation ideal.

(iv) $G$ is an ordered Abelian group, isomorphic to $(K-\{0\}) /(R-I)$, considering the latter as a multiplicative group.

$G$ is called the valuation group. 
(v) $v$ is the canonical map from $(K-\{0\})$, also denoted $K^{\#}$, to $G$.

$v$ is called the valuation.

(vi) $\bar{K}$ is a field isomorphic to $R / I$.

$\bar{K}$ is called the residue class field.

Proposition 1.23. If $(K, R, I, G, v, \bar{K})$ is a valued field, then for any two non-zero elements $a, b \in K$,

$$
v(a+b) \geq \min (v(a), v(b)) .
$$

Proof. Without loss of generality, assume that $v(a) \geq v(b)$. This is equivalent to $a / b \in R$. But then $(a / b)+1=(a+b) / b \in R$ and $v(a+b) \geq v(b)$.

DEFINITION 1.24. A valued field with cross-section is a pair $((K, R, I, G, v, \bar{K}) ; \pi)$ where $(K, R, I, G, v, \bar{K})$ is a valued field and $\pi$ is a group homomorphism from $G$ to $K^{\#}$ such that

$$
v(\pi(\alpha))=\alpha \text { for all } \alpha \in G \text {. }
$$

Definition 1.25. Given a valued field $(K, R, I, G, v, \bar{K})$, the residue map is the ring homomorphism $\rho: R \rightarrow K$, whose kernel is $I$. We write $\rho(x)=\bar{x}$.

DEFINITION 1.26. A Hensel field is a valued field $(K, R, I, G, v, \bar{K})$ with the following property:

Given a polynomial $P \in R[x]$, if $\bar{P} \in \bar{K}[x]$ has a non-singular root at $\alpha \in \bar{K}$, then $P$ has a root at some $a \in R$ such that $\bar{a}=\alpha$.

Definition 1.27. A valued field $(K, R, I, G, v, \bar{K})$ is $\omega$-pseudocomplete if: given a sequence $\left\{a_{j}\right\}, a_{j} \in K$, such that for all $j$,

$$
v\left(a_{j+2}-a_{j+1}\right)>v\left(a_{j+1}-a_{j}\right)
$$

then there exists $a \in K$ such that

$$
v\left(a-a_{j+1}\right)>v\left(a_{j+1}-a_{j}\right)
$$

for all $j \in \mathbf{N}$.

Definition 1.28. Given two valued fields $(K, R, I, G, v, \bar{K})$ and $\left(K^{\prime}, R^{\prime}, I^{\prime}, G^{\prime}, v^{\prime}, \bar{K}^{\prime}\right)$, an analytic isomorphism is a pair $\left(\psi, \psi^{\#}\right)$ where

(i) $\psi: K \rightarrow K^{\prime}$ is a field isomorphism.

(ii) $\psi^{\#}: G \rightarrow G^{\prime}$ is an Abelian group isomorphism.

(iii) For all $b \in K, b \neq 0, v^{\prime}(\psi(b))=\psi^{\#}(v(b))$. 
DeFINITION 1.29. Given two fields with cross section

$$
((K, R, I, G, v, \bar{K}) ; \pi) \text { and }\left(\left(K^{\prime}, R^{\prime}, I^{\prime}, G^{\prime}, v^{\prime}, \bar{K}^{\prime}\right) ; \pi^{\prime}\right)
$$

a cross-analytic isomorphism is an analytic isomorphism $\left(\psi, \psi^{\#}\right)$ such that

$$
\psi(\pi(\alpha))=\pi^{\prime}\left(\psi^{\#}(\alpha)\right), \quad \text { for all } \alpha \in G .
$$

Definition 1.30. A subgroup $H$ of a group $G$ is a pure subgroup if:

If $a \in H$ and $b \in G$ such that for some $n \in \mathbf{N}, n b=a$, then $b \in H$.

REMARK 1.31. If $G$ is a divisible group, then its subgroup $H$ is pure if and only if it is divisible.

DEFINITION 1.32. Let

$$
((K, R, I, G, v, \bar{K}) ; \pi) \text { and }\left(\left(K^{\prime}, R^{\prime}, I^{\prime}, G^{\prime}, v^{\prime}, \bar{K}^{\prime}\right) ; \pi^{\prime}\right)
$$

be two valued fields with cross section. Let $E$ be a subfield of $K$ and let $E^{\prime}$ be a subfield of $K^{\prime}$. A cross-analytic isomorphism $\left(\psi, \psi^{\#}\right)$ from $\left(\left(E, E \cap R, E \cap I, v(E),\left.v\right|_{E}, \bar{E}\right) ;\left.\pi\right|_{v(E)}\right)$ to $\left(\left(E^{\prime}, E^{\prime} \cap R^{\prime}\right.\right.$, $\left.\left.E^{\prime} \cap I^{\prime}, v^{\prime}\left(E^{\prime}\right),\left.v^{\prime}\right|_{E^{\prime}}, \bar{E}^{\prime}\right) ;\left.\pi^{\prime}\right|_{v^{\prime}\left(E^{\prime}\right)}\right)$ is a pure map if $v(E)$ is a countable pure subgroup of $G$ and $v^{\prime}\left(E^{\prime}\right)$ is a countable pure subgroup of $G^{\prime}$.

Proposition 1.33. Let $(K, R, I, G, v, \bar{K})$ and $\left(K^{\prime}, R^{\prime}, I^{\prime}, G^{\prime}\right.$, $\left.v^{\prime}, \bar{K}^{\prime}\right)$ be two valued fields. Then the following are equivalent:

(1) There exists an analytic isomorphism from $(K, R, I, G, v, \bar{K})$ to $\left(K^{\prime}, R^{\prime}, I^{\prime}, G^{\prime}, v^{\prime}, \bar{K}^{\prime}\right)$.

(2) $R \simeq R^{\prime}$.

(3) $I \simeq I^{\prime}$.

Proof. That $(1) \rightarrow(2) \rightarrow(3)$ is trivial. It remains to show that $(3) \rightarrow(1)$. Let $\theta: I \rightarrow I^{\prime}$ be the isomorphism. We will define an isomorphism $\psi: K \rightarrow K^{\prime}$. Let $\alpha \in K$; if $1 / \alpha \in I$, then $\psi(\alpha)=$ $1 /(\theta(1 / \alpha))$. If $1 / \alpha \notin I$, then for $\beta \in I$, we have that $(\alpha \beta) \in I$, so for $\beta \neq 0$ let $\psi(\alpha)=(\theta(\alpha \beta)) / \theta(\beta)$. Then, $\psi(R)=R^{\prime}$ and $\psi(R-I)=R^{\prime}-I^{\prime}$. Consider an element of $G,[\alpha \cdot(R-I)]$. We define $\psi^{\#}, \psi^{\#}([\alpha \cdot(R-I)])=\left[\psi(\alpha) \cdot\left(R^{\prime}-I^{\prime}\right)\right]$. This completes the proof.

Because of Proposition 1.33 we have the following: 
DEFINITION 1.34. Let $R$ be a domain (but not a field) such that, for all $x, y \in R$, either $x \mid y$ or $y \mid x$. Then letting $I$ be the set of non-units of $R$, we have a valued field $(\operatorname{Frac}(R), R, I$, $\left.\operatorname{Frac}(R)^{\#} /(R-I), v, R / I\right)$. We call this valued field the valued field generated by $R$.

LeMma 1.35. Let $K$ be a field. Let $I \subset K$ be a subset such that

(a) $I$ is closed under addition, subtraction, and multiplication.

(b) $K-I$ is closed under multiplication.

(c) $1 \notin I$.

Then $I$ determines a valued field, the valued field generated by $R=$ $\{x \in K \mid 1 / x \notin I\}$.

Proof. Given $I \subset K, R$ is unique, and $K$ is the fraction field of $R, \operatorname{Frac}(R)$. We need to show that $R$ is closed under addition. Let $(a+b) \in K-R$, that is, $1 /(a+b) \in I$. We shall show that either $a$ or $b$ is in $K-R$, that is, either $1 / a$ or $1 / b$ is in $I$. But $1 /(a+b)=$ $(1 / b)-(a / b) \cdot(1 /(a+b))$ and $1 /(a+b)=(1 / a)-(b / a) \cdot(1 /(a+b))$. Recall that $I$ is closed under addition, so if $((a / b) \cdot(1 /(a+b))) \in I$ then so is $1 / b$, similarly for $(b / a) \cdot(1 /(a+b))$ and $1 / a$. But

$$
((a / b):(1 /(a+b))) \cdot((b / a) \cdot(1 /(a+b)))=1 /\left((a+b)^{2}\right) \in I .
$$

Therefore $R$ is closed under addition (recall that $K-I$ is multiplicatively closed), and the proof is complete.

NotAtion 1.36 . Because of Lemma 1.35 , we can identify a valued field by identifying the main field $K$ and the valuation ideal $I$. Thus, $\left(K=\operatorname{Frac}(c / p), I=c_{0} / p\right)$ will denote the unique valued field with main field $\operatorname{Frac}(c / p)$ and valuation ideal $I=c_{0} / p$. Similarly, we shall let $R=l^{\infty} / p$ denote the unique valued field having valuation ring $l^{\infty} / p$.

D. Ordered divisible Abelian groups. We can capsulize the AxKochen results by saying that under certain conditions two valued fields are isomorphic if their residue class fields are isomorphic and their valuation groups are isomorphic.

For all valued fields that we deal with in this paper the valuation groups will be ordered divisible Abelian groups.

Definition 1.37. An ordered divisible Abelian group is an ordered Abelian group $G$, such that for all $b \in G$ and $n \in N$, there exists $c \in G$ such that $n c=b$. 
REMARK 1.38. A torsion free divisible Abelian group is a vector space over $\mathbf{Q}$. A fortiori, an ordered divisible Abelian group is a vector space over $\mathbf{Q}$.

Remark 1.39. Let $G$ and $H$ be two ordered divisible Abelian groups and let $f: G \rightarrow H$ be surjective. Then there exists a homomorphism $g: H \rightarrow G$ such that $f \circ g: H \rightarrow H$ is the identity. In particular, for a valued field $(K, R, I, G, v, \bar{K})$, if $K^{\#} /\{-1,1\}$ and $G$ are divisible groups, then there exists a cross-section $\pi: G \rightarrow K^{\#}$.

Definition 1.40. An ordered divisible Abelian group $G$ is $\omega_{1^{-}}$. saturated if it satisfies the following property:

Let $A$ and $B$ be two subsets of $G$ such that for all $a \in A$ and $b \in B, a<b$. Let card $A \leq \aleph_{0}$ and card $B \leq \aleph_{0}$. Then there exists $g \in G$ such that

(i) $a<g$ for all $a \in A$ and

(ii) $g<b$ for all $b \in B$.

We observe that either $A$ or $B$ (or both) may be finite or empty.

Proposition 1.41. Let $G$ and $H$ be two ordered divisible Abelian groups. Let $\operatorname{card} G=\operatorname{card} H=\aleph_{1}$. Let both $G$ and $H$ be $\omega_{1}$ saturated. Then there exists an ordered Abelian group isomorphism $\theta: G \rightarrow H$.

Proof. We prove this by a very standard method. First we well order the elements of $G$ and $H,\left\{g_{\alpha}\right\}_{\alpha<\omega_{1}}$ and $\left\{h_{\alpha}\right\}_{\alpha<\omega_{1}}$. (Of course, this well ordering has no connection with the orders on either group.) For each $g_{\beta} \in G$, we have two sets

$$
g_{\beta}^{U}=\left\{g \in G \mid g>g_{\beta}\right\} \quad \text { and } \quad g_{\beta}^{L}=\left\{g \in G \mid g<g_{\beta}\right\},
$$

similarly for $h_{\beta} \in H$.

We shall construct a family of isomorphisms between subgroups

$$
\left\{\theta_{\alpha}: G_{\alpha} \rightarrow H_{\alpha}\right\}_{\alpha<\omega_{1}}
$$

such that

(a) for all $\gamma>\alpha, G_{\alpha} \subset G_{\gamma}, H_{\alpha} \subset H_{\gamma}$, and $\theta_{\gamma}$ extends $\theta_{\alpha}$ and

(b) $G=\bigcup_{\alpha<\omega_{1}} G_{\alpha}, H=\bigcup_{\alpha<\omega_{1}} H_{\alpha}$, and such that $\theta=\bigcup_{\alpha<\omega_{1}} \theta_{\alpha}$ is the desired isomorphism.

First, we let $G_{0}=\{0\}, H_{0}=\{0\}$, and $\theta_{0}(0)=0$.

For a limit ordinal $\lambda$, we let $G_{\lambda}=\bigcup_{\alpha<\lambda} G_{\alpha}, H_{\lambda}=\bigcup_{\alpha<\lambda} H_{\alpha}, \theta_{\lambda}=$ $\bigcup_{\alpha<\lambda} \theta_{\alpha}$. 
If $\alpha$ is of the form $\alpha=\lambda+2 n-1$, we choose $g_{\beta} \notin G_{\alpha-1}$ such that $\beta \leq \gamma$ for all $g_{\gamma} \notin G_{\alpha-1}$. Then $G_{\alpha}$ will be the subgroup generated by $g_{\beta}$ and $G_{\alpha-1}$. For $\theta_{\alpha}\left(g_{\beta}\right)$ we choose $h \in H$ such that

(i) for all $g \in G_{\alpha-1} \cap g_{\beta}^{U}, \theta_{\alpha-1}(g)>h$,

(ii) for all $g \in G_{\alpha-1} \cap g_{\beta}^{L}, \theta_{\alpha-1}(g)<h$.

Since $G_{\alpha-1}$ is countable, the existence of such an $h$ is guaranteed by hypothesis.

Finally, if $\alpha$ is of the form $\alpha=\lambda+2 n$, we choose $h_{\beta} \notin H_{\alpha-1}$ such that $\beta \leq \gamma$ for all $h_{\gamma} \notin H_{\alpha-1}$. For $\theta_{\alpha}^{-1}\left(h_{\beta}\right)$ we choose $g \in G$, such that

(1) for $h \in H_{\alpha-1} \cap h_{\beta}^{U}, \theta_{\alpha-1}^{-1}(h)>g$,

(2) for $h \in H_{\alpha-1} \cap h_{\beta}^{L}, \theta_{\alpha-1}^{-1}(h)<g$.

This completes the construction and the proof.

Proposition 1.42. Let $G$ be an $\omega_{1}$-saturated ordered divisible Abelian group. Let $H$ be a convex subgroup of $G$, and let $H$ have $a$ countable cofinal subset. Then $G / H$ is an $\omega_{1}$-saturated ordered divisible Abelian group.

Proof. Clearly $G / H$ is a divisible Abelian group. Since $H$ is a convex subset of $G, G / H$ inherits the order from $G$. To see that $G / H$ is $\omega_{1}$-saturated, let $A=\left\{\left[a_{j}+H\right]\right\}$ and $B=\left\{\left[b_{k}+H\right]\right\}$. As usual, let $\left[a_{j}+H\right]<\left[b_{k}+H\right]$ for all $a_{j}$ and $b_{k}$. Let $\left\{h_{m}\right\}$ be the countable cofinal subset of $H$, with $h_{m}>0$ for all $m$. We then have two countable subsets of $G$

$$
A_{1}=\left\{\left(a_{j}+h_{m}\right)\right\}_{j, m \in \mathbf{N}^{\prime}}, \quad B_{1}=\left\{\left(b_{k}-h_{n}\right)\right\}_{k, n \in \mathbf{N}^{\prime}}
$$

and $a_{j}+h_{m}<b_{k}-h_{n}$, for all $j, k, m$, and $n$. Then, by $\omega_{1^{-}}$ saturation of $G$ there exists $g \in G$, such that

(i) $a_{j}+h_{m}<g$ for all $a_{j}$ and $h_{m}$,

(ii) $b_{k}-h_{n}>g$ for all $b_{k}$ and $h_{n}$.

Therefore

$$
\left[a_{j}+H\right]<[g+H] \text { for all }\left[a_{j}+H\right] \in A
$$

and

$$
\left[b_{k}+H\right]>[g+H] \text { for all }\left[b_{k}+H\right] \in B .
$$

2. Residue class domains of $c$. In this section we shall show that there are either exactly 7 or exactly 10 residue class domains of $c$. In $\S 3$ we shall show that there are exactly 10 . 
2.1. $c$ and $l^{\infty}$. In this part of $\S 2$, we shall show four things:

1. $\operatorname{Frac}(c / p)=\operatorname{Frac}\left(l^{\infty} / p\right)$.

2. $\operatorname{Frac}(c / p)$ can be made a valued field with residue class field $\operatorname{Frac}\left(l^{\infty} / c_{0}\right)$. We also classify all the valuation groups of these valued fields. There are three.

3. $\operatorname{Frac}\left(l^{\infty} / c_{0}\right)$ can be made a valued field with residue class field $\mathbf{R}$. We classify all possible valuation groups for these valued fields. There are at most three, including the trivial $\{0\}$.

4 . We show that the valued fields in 2 and 3 are $\omega$-pseudo-complete. $\omega$-pseudo-completeness is one of the hypotheses of the Ax-Kochen Theorem. 1 is needed to show 2 and 3.

In part 2 of this section we shall apply Ax-Kochen machinery to 2 and 3 to show that there are either exactly 7 or exactly 10 residue class domains of $c$.

Recall that to every non-maximal prime ideal $p$ of $c$, there is associated a non-principal ultrafilter $\mathscr{U}$ on $\mathbf{N}$ such that $Z(f) \in \mathscr{U}$ implies $f \in p$.

Observe also:

REMARK 2.1.1. Let $\mathscr{U}$ be a non-principal ultrafilter on $\mathbf{N}$. For $f, g \in c$, if

$$
f \underset{\mathscr{U}}{>} g \underset{\mathscr{U}}{>} 0
$$

then there exists $\theta \in l^{\infty}$ such that

$$
f \theta \underset{\overline{\bar{U}}}{ } g .
$$

On the other hand, we shall see later that there exist an ultrafilter $\mathscr{V}$ and $f, g \in c$ such that

$$
f \underset{\mathscr{V}}{>} g \underset{\mathscr{V}}{>} 0
$$

and

$$
f \theta \underset{\overline{\mathscr{V}}}{\equiv} \quad \text { implies } \quad \theta \notin c
$$

( $f$ and $g$, of course, depend on $\mathscr{V}$.) Therefore, $l^{\infty}$ will play a major role in this paper.

Notation 2.1.2. Let $p$ be a non-maximal prime ideal of $c$, and 
let $\mathscr{U}$ be the ultrafilter associated with $p$. Then

$$
\begin{aligned}
p_{b} & =\left\{\theta \in l^{\infty} \mid \exists f \in p ; f \overline{\overline{\mathscr{U}}} \theta\right\}, \\
c_{0, \mathscr{U}} & =\left\{\theta \in l^{\infty} \mid \exists f \in c_{0} ; f \overline{\overline{\overline{\mathscr{U}}}} \theta\right\}, \\
l_{0, \mathscr{U}} & =\left\{\theta \in l^{\infty} \mid \lim _{\mathscr{U}} \theta=0\right\} .
\end{aligned}
$$

REMARK 2.1.3. $p_{b}, c_{0, \mathscr{U}}$, and $l_{0, \mathscr{U}}$ are all convex prime ideals of $l^{\infty} . l_{0, \mathscr{U}}$ is a maximal ideal.

We shall show that $\operatorname{Frac}(c / p)$ and $c_{0} / p$ generate a valued field $\left(K=\operatorname{Frac}(c / p), I=c_{0} / p\right)$. Further, the residue class field of the above mentioned value field is isomorphic to $\operatorname{Frac}\left(l^{\infty} / c_{0}, \mathscr{U}\right)$. Finally, Frac $\left(l^{\infty} / c_{0, \mathscr{U}}\right)$ and $l_{0, \mathscr{U}} / c_{0, \mathscr{U}}$ also generate a valued field $(K=$ $\left.\operatorname{Frac}\left(l^{\infty} / c_{0}, \mathscr{U}\right), I=l_{0, \mathscr{U}} / c_{0}, \mathscr{U}\right)$, and the residue class field of this field is $\mathbf{R}$.

We would like first to characterize $\operatorname{Frac}(c / p)$. We do so in the next proposition.

Proposition 2.1.4. Let $p$ be a non-maximal prime ideal of $c$. Then

$$
\operatorname{Frac}(c / p) \simeq \operatorname{Frac}\left(l^{\infty} / p_{b}\right) \text {. }
$$

Proof. We have the field isomorphism induced by the map $i: c / p \rightarrow$ $l^{\infty} / p_{b}$ where $i([f+p])=\left[f+p_{b}\right]$. We need to show that this field isomorphism is onto. So let

$$
\frac{\left[\theta+p_{b}\right]}{\left[\psi+p_{b}\right]} \in \operatorname{Frac}\left(l^{\infty} / p_{b}\right)
$$

with $\theta \in l^{\infty}$ and $\psi \in l^{\infty}$. Let $g \in c_{0}-p$. Then $(g \theta) \in c_{0}$. Hence

$$
i\left(\frac{[g \theta+p]}{[g \psi+p]}\right)=\frac{\left[g \theta+p_{b}\right]}{\left[g \psi+p_{b}\right]}=\frac{\left[\theta+p_{b}\right]}{\left[\psi+p_{b}\right]} \text {. }
$$

Next we shall classify a special case of $\operatorname{Frac}(c / p)$, namely the case where $p=p_{\mathscr{U}}$.

Proposition 2.1.5. For a non-principal ultrafilter $\mathscr{U}$ on $\mathbf{N}$, let

$$
p_{\mathscr{U}}=\{f \in c \mid Z(f) \in \mathscr{U}\} .
$$

Then

$$
\operatorname{Frac}\left(c / p_{\mathscr{U}}\right) \simeq \operatorname{Frac}\left(l^{\infty} / p_{\mathscr{U}, b}\right) \simeq\left(\prod_{\mathbf{N}} \mathbf{R}\right) / \mathscr{U}
$$


Proof. By Proposition 2.1.4, we need only show that $\operatorname{Frac}\left(l^{\infty} / p_{\mathscr{U}, b}\right)$ is isomorphic to $\left(\prod_{\mathbf{N}} \mathbf{R}\right) / \mathscr{U}$. Certainly, $l^{\infty} \subset \prod_{\mathbf{N}} \mathbf{R}$, and $\theta \equiv_{\mathscr{U}} \psi$ is equivalent to $(\theta-\psi) \in p_{\mathscr{U}, b}$, so $\operatorname{Frac}\left(l^{\infty} / p_{\mathscr{U}, b}\right) \subset\left(\prod_{\mathbf{N}}^{\mathbf{N}} \mathbf{R}\right) / \mathscr{U}$. Next, let $\Omega \in \prod_{\mathbf{N}} \mathbf{R}$. If $1 \geq_{\mathscr{U}}|\Omega|$, then

$$
\Omega \underset{\overline{\mathscr{U}}}{\overline{1}} 1 \wedge \Omega \vee(-1)=\Omega_{1}
$$

and $\Omega_{1} \in l^{\infty}$. If $\Omega>_{\mathscr{U}} 1$, let $\Omega_{2}=\Omega \vee 1 \equiv_{\mathscr{U}} \Omega$. If $\Omega<_{\mathscr{U}}-1$, let $\Omega_{2}=\Omega \wedge(-1) \equiv_{\mathscr{U}} \Omega$. Then,

$$
\frac{1}{\Omega_{2}} \in l^{\infty} .
$$

We wish to show now that, for a non-maximal prime ideal $p$, $\operatorname{Frac}(c / p)$ and $c_{0} / p$ generate a valued field $(K=\operatorname{Frac}(c / p), I=$ $\left.c_{0} / p\right)$. This will be done in Proposition 2.1.8.

LEMMA 2.1.6. Let $p$ be a non-maximal prime ideal for $c$. Then $l^{\infty} / p_{b}$ is a convex subring of $\operatorname{Frac}\left(l^{\infty} / p_{b}\right)$.

Proof. Let $\left|\left[\theta+p_{b}\right]\right|>\left|\left[\psi+p_{b}\right]\right|$. Then $|\psi| \equiv_{\mathscr{U}}|\theta| \wedge|\psi|$. Clearly $|\theta(n)| \geq|\theta(n)| \wedge|\psi(n)|$ for all $n \in \mathbf{N}$, so

$$
\frac{|\theta| \wedge|\psi|}{\theta} \in l^{\infty}
$$

hence

$$
\frac{\left|\left[\psi+p_{b}\right]\right|}{\left[\theta+p_{b}\right]} \in l^{\infty} / p_{b}
$$

and

$$
\frac{\left[\psi+p_{b}\right]}{\left[\theta+p_{b}\right]} \in l^{\infty} / p_{b}
$$

LEMMA 2.1.7. Let $p$ be a non-maximal prime ideal of $c$. Then

$$
\left[\operatorname{Frac}(c / p)-c_{0} / p\right]
$$

is a multiplicatively closed subset of $\operatorname{Frac}(c / p)$.

Proof. This is clearly equivalent to $\left[\operatorname{Frac}\left(l^{\infty} / p_{b}\right)-c_{0, \mathscr{U}} / p_{b}\right]$ being multiplicatively closed. By Lemma 2.1.6 and Remark 2.1.3, $c_{0, \mathscr{U}} / p_{b}$ is convex in $\operatorname{Frac}\left(l^{\infty} / p_{b}\right)$. Let

$$
\frac{\left[\theta_{1}\right]}{\left[\psi_{1}\right]} \text { and } \frac{\left[\theta_{2}\right]}{\left[\psi_{2}\right]}
$$


be elements of $\operatorname{Frac}\left(l^{\infty} / p_{b}\right)$. Assume

$$
\frac{\left[\theta_{1}\right]}{\left[\psi_{1}\right]} \cdot \frac{\left[\theta_{2}\right]}{\left[\psi_{2}\right]} \in c_{0, \mathscr{U}} / p_{b}
$$

Assume towards contradiction that

$$
\frac{\left[\theta_{1}\right]}{\left[\psi_{1}\right]} \notin c_{0, \mathscr{U}} / p_{b}
$$

and

$$
\frac{\left[\theta_{2}\right]}{\left[\psi_{2}\right]} \notin c_{0, \mathscr{U}} / p_{b} .
$$

By convexity of $c_{0, \mathscr{U}} / p_{b}$, this implies that both

$$
\left|\frac{\left[\theta_{1}\right]}{\left[\psi_{1}\right]}\right| \text { and }\left|\frac{\left[\theta_{2}\right]}{\left[\psi_{2}\right]}\right|
$$

are greater than their product. Hence

$$
1>\left|\frac{\left[\theta_{2}\right]}{\left[\psi_{2}\right]}\right| \quad \text { and } \quad 1>\left|\frac{\left[\theta_{1}\right]}{\left[\psi_{1}\right]}\right| \text {. }
$$

Therefore

$$
\frac{\left[\theta_{1}\right]}{\left[\psi_{1}\right]} \in l^{\infty} / p_{b} \text { and } \frac{\left[\theta_{2}\right]}{\left[\psi_{2}\right]} \in l^{\infty} / p_{b}
$$

Since $c_{0, \mathscr{U}} / p_{b}$ is a prime ideal of $l^{\infty} / p_{b}$, the result follows.

Proposition 2.1.8. For $p$ a non-maximal prime ideal of $c$, $\operatorname{Frac}(c / p)$ and $c_{0} / p$ generate a valid field $\left(K=\operatorname{Frac}(c / p), I=c_{0} / p\right)$.

\section{Proof. Lemmas 2.1.7 and 1.35.}

We wish now to study the valuation group of the valued field $(K=$ $\left.\operatorname{Frac}(c / p), I=c_{0} / p\right)$. We shall show that it is always a convex subgroup of an $\omega_{1}$-saturated ordered divisible Abelian group. This is done in Proposition 2.1.12.

LEMMA 2.1.9. Let $\left\{\left[f_{m}\right]\right\}$ be a countable subset of $c_{0} / p$ (or of $\left.l_{0, \mathscr{U}} / p_{b}\right)$. Then there exists $[g] \in c_{0} / p\left(\right.$ or $[g] \in l_{0, \mathscr{U}} / p_{b}$, respectively) such that $[g]>\left|\left[f_{m}\right]\right|$ for all $m$.

Proof. Given $\left\{f_{m}\right\}$, we define $\left\{h_{j}\right\}$ :

$$
h_{j}=\left|f_{1}\right| \vee\left|f_{2}\right| \vee \cdots \vee\left|f_{j}\right| \text {. }
$$

Clearly $h_{j} \equiv_{\mathscr{U}}\left|f_{j}\right|$. 
We next define a function $k: \mathbf{N} \rightarrow \mathbf{N} \cup\{\infty\}:$

$$
k(n)=\sup \left\{j \mid 1 \wedge h_{j}(n) \leq 1 / j\right\} .
$$

We next define $g: \mathbf{N} \rightarrow \mathbf{R}$, by

$$
g(n)= \begin{cases}\frac{1}{k(n)} & \text { if } k(n) \in \mathbf{N} \\ 0 & \text { if } k(n)=\infty\end{cases}
$$

It can be checked that $g$ has the desired properties.

REMARK 2.1.10. If $v$ is the valuation in the valued field ( $K=$ $\left.\operatorname{Frac}(c / p), I=c_{0} / p\right)$, then $v([f])>v([g])$ if and only if $[f] /[g] \in$ $c_{0} / p$.

LEMMA 2.1.11. Let $\mathscr{U}$ be a non-principal ultrafilter on $\mathbf{N}$. Let $\left\{f_{m}\right\}$ and $\left\{g_{k}\right\}$ be two countable subsets of $l_{0, \mathscr{U}}$ (either or both may be finite or empty), such that $f_{m}>_{\mathscr{U}} g_{k}$ for all $m$ and $k$. Then there exists $h \in l_{0, \mathscr{U}}$ such that

$$
f_{m}>_{\mathscr{U}} h>_{\mathscr{U}} g_{k} \text { for all } m \text { and } k .
$$

We leave the proof of Lemma 2.1.11 to the reader.

Proposition 2.1.12. Let $p$ be a non-maximal prime ideal of $c$. Let $\left\{\left[f_{m}\right]\right\}$ and $\left\{\left[g_{k}\right]\right\}$ be countable subsets of $c_{0} / p$ (either or both subsets may be finite and $\left\{\left[f_{m}\right]\right\}$ may be empty) such that

$$
\left[f_{m}\right]>\left[g_{k}\right]>0
$$

and

$$
\frac{\left[g_{k}\right]}{\left[f_{m}\right]} \in c_{0} / p \text { for all } m \text { and } k .
$$

Let the set $\left\{\left[g_{k}\right]\right\}$ be non-empty. Then there exists $[h] \in c_{0} / p$ such that

$$
\frac{[h]}{\left[f_{m}\right]} \in c_{0} / p \text { for all } m
$$

and

$$
\frac{\left[g_{k}\right]}{[h]} \in c_{0} / p \text { for all } k .
$$

Proof. We consider four cases.

Case 1. $\left\{\left[f_{m}\right]\right\}$ is empty. This is Lemma 2.1.9.

Case 2. For each $\left[f_{k}\right]$ there exists $j>k$ such that

$$
\frac{\left[f_{j}\right]}{\left[f_{k}\right]} \in c_{0} / p
$$


and for each $\left[g_{m}\right]$ there exists $i>m$ such that

$$
\frac{\left[g_{m}\right]}{\left[g_{i}\right]} \in c_{0} / p
$$

In this case, let $h$ be the sequence guaranteed by Lemma 2.1.11,

$$
f_{k}>_{\mathscr{U}} h>_{\mathscr{U}} g_{m} \text { for all } m \text { and } k \text {. }
$$

Then, since $f_{j}>_{\mathscr{U}} h$, it follows that

$$
\frac{\left[f_{j}\right]}{\left[f_{k}\right]}>\frac{[h]}{\left[f_{k}\right]} \in c_{0} / p
$$

Since $h>_{\mathscr{U}} g_{i}$, it follows that

$$
\frac{\left[g_{m}\right]}{\left[g_{i}\right]}>\frac{\left[g_{m}\right]}{[h]} \in c_{0} / p
$$

Case 3. For each $\left[f_{k}\right]$, there exists $j>k$ such that

$$
\frac{\left[f_{j}\right]}{\left[f_{k}\right]} \in c_{0} / p
$$

however, there exists $\left[g_{i}\right]$ such that for all $\left[g_{m}\right]$,

$$
\frac{\left[g_{i}\right]}{\left[g_{m}\right]} \notin c_{0} / p
$$

(or vice versa for $f$ and $g$ ). In this case, let

$$
\frac{\left[g_{i}\right]}{\left[f_{k}\right]}=\left[\theta_{k}\right] \in c_{0} / p
$$

By Lemma 2.1.9, there exists $[\theta] \in c_{0} / p$ such that $[\theta]>\left[\theta_{k}\right]$ for all $k$. Let

$$
[h]=\frac{\left[g_{i}\right]}{[\theta]} .
$$

Clearly $\left[f_{j}\right]>[h]$ for all $j$, and hence

$$
\frac{\left[f_{j}\right]}{\left[f_{k}\right]}>\frac{[h]}{\left[f_{k}\right]} \in c_{0} / p \quad \text { for all } k
$$

Clearly

$$
\frac{\left[g_{i}\right]}{[h]}=[\theta] \in c_{0} / p,
$$

and for $m>i$,

$$
\frac{\left[g_{i}\right]}{[h]}=\frac{\left[g_{m}\right]}{[h]} \cdot \frac{\left[g_{i}\right]}{\left[g_{m}\right]} \in c_{0} / p,
$$

but

$$
\frac{\left[g_{i}\right]}{\left[g_{m}\right]} \notin c_{0} / p
$$


therefore, by primality of $c_{0} / p$,

$$
\frac{\left[g_{m}\right]}{[h]} \in c_{0} / p .
$$

Case 4. There exist $\left[f_{j}\right]$ and $\left[g_{i}\right]$ such that

$$
\frac{\left[f_{k}\right]}{\left[f_{j}\right]} \notin c_{0} / p \quad \text { for all } k
$$

and

$$
\frac{\left[g_{i}\right]}{\left[g_{m}\right]} \notin c_{0} / p \text { for all } m \text {. }
$$

In this case, let $[h]=\sqrt{\left[f_{j}\right] \cdot\left[g_{i}\right]}$. Clearly,

$$
\frac{[h]}{\left[f_{j}\right]}=\frac{\left[g_{i}\right]}{[h]}=\sqrt{\frac{\left[g_{i}\right]}{\left[f_{j}\right]}} \in c_{0} / p .
$$

For $k \in \mathbf{N}$ such that $\left[f_{j}\right]>\left[f_{k}\right]$,

$$
\frac{[h]}{\left[f_{k}\right]} \cdot \frac{\left[f_{k}\right]}{\left[f_{j}\right]}=\frac{[h]}{\left[f_{i}\right]} \in c_{0} / p,
$$

and by primality of $c_{0} / p$,

$$
\frac{[h]}{\left[f_{k}\right]} \in c_{0} / p .
$$

For $m \in \mathbf{N}$ such that $\left[g_{m}\right]>\left[g_{i}\right]$,

$$
\frac{\left[g_{m}\right]}{[h]} \cdot \frac{\left[g_{i}\right]}{\left[g_{m}\right]}=\frac{\left[g_{i}\right]}{[h]} \in c_{0} / p
$$

and by primality of $c_{0} / p$,

$$
\frac{\left[g_{m}\right]}{[h]} \in c_{0} / p
$$

If $p$ is a prime ideal of $c$, such that $p_{\mathscr{U}} \subset p \subset c_{0}$, then the valuation group of the valued field $\left(K=\operatorname{Frac}(c / p), I=c_{0} / p\right)$ is a convex subgroup of the valuation group of $\left(K=\operatorname{Frac}\left(c / p_{\mathscr{U}}\right), I=c_{0} / p_{\mathscr{U}}\right)$. Accordingly, we turn our attention to the case $p=p_{\mathscr{U}}$.

LEMMA 2.1.13. Let $\mathscr{U}$ be a non-principal ultrafilter on $\mathbf{N}$ and let $\left\{f_{j}\right\}$ be a countable family of sequences such that

$$
f_{j} \underset{\mathscr{U}}{>} f_{j+1} \underset{\mathscr{U}}{>} 0 .
$$

Then there exists $g$ such that

$$
f_{j} \underset{\mathscr{U}}{>} g \underset{\mathscr{U}}{>} 0 \text { for all } j .
$$


Proof. First we define a family of sequences $\left\{g_{j}\right\}$, by

$$
g_{j}(n)= \begin{cases}f_{j}(n) & \text { if } f_{j}(n)>0, \\ 1 / n & \text { if } f_{j}(n) \leq 0\end{cases}
$$

Then let $g(n)=g_{1}(n) \wedge \cdots \wedge g_{n}(n)$.

Proposition 2.1.14. If $p=p_{\mathscr{U}}$ for some non-principal ultrafilter $\mathscr{U}$, then the values field $(K=\operatorname{Frac}(c / p), I=c / p)$ has an $\omega_{1}$-saturated valuation group.

Proof. Follows by Lemma 2.1.13, Proposition 2.1.12, and Lemma 2.1.9.

We next show that the valuation group of $(K=\operatorname{Frac}(c / p), I=$ $\left.c_{0} / p\right)$ must take one of three forms. We shall later show (Theorem 2.1.17) that each of these three forms describes a unique group up to isomorphism.

THEOREM 2.1.15. Let $p$ be a non-maximal prime ideal of $c$. Let $G$ be the valuation group of the valued field $\left(K=\operatorname{Frac}(c / p), I=c_{0} / p\right)$. Then $G$ has one of the following forms:

(i) $G$ is $\omega_{1}$-saturated.

(ii) $G=\mathbf{R} \oplus G_{0}$ with lexicographical ordering, and with $G_{0}$ being an $\omega_{1}$-saturated group.

(iii) $G=\bigcup_{j=1}^{\infty}\left(\mathbf{R}_{j} \oplus G_{j}\right)$ where each sum $\mathbf{R}_{j} \oplus G_{j}$ is as in (ii), and $\mathbf{R}_{j} \oplus G_{j}$ is a convex subgroup of $G_{j+1}$.

Proof. A cofinal subset of $G$ is the image of a coinitial subset of $\left(c_{0} / p\right)_{+}$.

If $\left(c_{0} / p\right)_{+}$has no countable coinitial subset, then by Lemma 2.1.9 and Proposition 2.1.12, $G$ is $\omega_{1}$-saturated. As an example of this case, we have $p=p_{\mathscr{U}}$.

If $\left(c_{0} / p\right)_{+}$has a countable coinitial subset, then either there exists $[f] \in c_{0} / p$ such that $\left\{[f]^{k}\right\}$ is coinitial or no such $[f]$ exists.

First, assume that such an $[f]$ exists. Let

$$
\widetilde{H}=\left\{\left.[\theta] \in \operatorname{Frac}(c / p)||[\theta]\right|^{k}>[f] \text { and }|[\theta]|^{-k}>[f] \text { for all } k\right\} \text {. }
$$

Claim. $G_{0}=v(\tilde{H})$ is an $\omega_{1}$-saturated ordered divisible Abelian group. 
Proof of claim. By Lemma 2.1.9 and Proposition 2.1.12, it suffices to show that $v(\widetilde{H})$ has no countable cofinal subset. This is equivalent to showing that $(\widetilde{H})_{+}$has no countable coinitial subset.

We assume toward a contradiction that $(\widetilde{H})_{+}$has a countable coinitial subset $\left\{\left[g_{j}\right]\right\}$. But by Proposition 2.1.12, there exists $[h] \in c_{0} / p$ such that $\left[g_{j}\right]>[h]>[f]^{1 / k}$ for $j \in \mathbf{N}$ and $k \in \mathbf{N}$. By definition of $\widetilde{H},[h] \in(\widetilde{H})_{+}$and so $\left\{\left[g_{j}\right]\right\}$ is not coinitial. This proves the claim.

Now, let $[g] \in\left(c_{0} / p\right)_{+}$. We define

$$
r([g])=\inf \left\{t \in \mathbf{R}^{+}||[g] \mid>[f]^{t}\right\}
$$

then

$$
\frac{[g]}{[f]^{r}} \in \widetilde{H}
$$

So

$$
v([g])=\left(r([g]), v\left(\frac{[g]}{[f]^{r}}\right)\right) \in \mathbf{R} \oplus G_{0} .
$$

As an example of this case we have, letting $f(n)=e^{-n}$, for any non-principal ultrafilter $\mathscr{U}$,

$$
p=\left\{g \mid \text { for all } k, f^{k} \underset{\mathscr{U}}{>}|g|\right\} \text {. }
$$

Finally, we assume that $\left(c_{0} / p\right)_{+}$has a countable coinitial subset but no $[f]$ such as above exists. It is trivial to show that in this case we have a countable coinitial subset $\left\{\left[f_{m}\right]\right\}_{m=1}^{\infty}$ where $\left[f_{m}\right]^{k}>\left[f_{m+1}\right]$ for all $k \in \mathbf{N}$ and $m \in \mathbf{N}$. If we let

$$
\widetilde{H}_{m}=\left\{\left.[\theta] \in \operatorname{Frac}(c / p)||[\theta]\right|^{k}>\left[f_{m}\right] \text { and }|1 /[\theta]|^{k}>\left[f_{m}\right]\right.
$$

$$
\text { for all } k \in \mathbf{N} \text {, }
$$

then it is easy to show that $v\left(\widetilde{H}_{m}\right)=G_{m}$ is an $\omega_{1}$-saturated ordered divisible Abelian group. Let $[g] \in\left(c_{0} / p\right)_{+}$. Then by coinitiality of $\left\{\left[f_{m}\right]\right\}_{m=1}^{\infty}$, there exists $\left[f_{j}\right]$ such that $\left\{t \in \mathbf{R}^{+} \mid[g]>\left[f_{j}\right]^{t}\right\}$ is nonempty. Let

$$
r_{j}([g])=\inf \left\{t \in \mathbf{R}^{+} \mid[g]>\left[f_{j}\right]^{t}\right\}
$$

Then

$$
\frac{[g]}{\left[f_{j}\right]^{r_{j}}} \in \widetilde{H}_{j}
$$

and

$$
v([g])=\left(r_{j}([g]), v\left(\frac{[g]}{[f]^{r}}\right)\right) \in \mathbf{R}_{j} \oplus G_{j} .
$$


As an example of this case we have, letting

$$
\begin{gathered}
f_{k}(n)=e^{-n^{h}}, \\
p=\left\{g\left|f_{k} \underset{\mathscr{U}}{>}\right| g \mid \text { for all } k\right\} .
\end{gathered}
$$

LEMMA 2.1.16. Let $G$ and $H$ be two ordered vector spaces over $\mathbf{R}$. Let card $G=$ card $H=\aleph_{1}$. Let both $G$ and $H$, considered as ordered divisible Abelian groups, be convex subgroups of $\omega_{1}$-saturated groups.

Let there exist $\left\{g_{n}\right\} \subset G$ such that for all $c \in G$, there exists $g_{n}$ such that $g_{n}>c$; and $g_{n+1}>r \cdot g_{n}$ for all $r \in \mathbf{R}$ and all $n \in \mathbf{N}$.

Let $\left\{h_{m}\right\}$ be an analogous subset of $H$.

Then $H$ and $G$ are isomorphic as ordered Abelian groups.

Proof. For each $g_{n}$, we define

(a) $G_{n, L}=\left\{c \in G\left|g_{n}>\right| r \cdot c \mid\right.$ for all $\left.r \in \mathbf{R}\right\}$.

(b) $G_{n, U}=\left\{c \in G \mid\right.$ there exists $r \in \mathbf{R}$ such that $\left.r g_{n}>c\right\}$. Also, for each $h_{m}$ we define $H_{m, L}$ and $H_{m, U}$, in the same fashion.

We observe that, for all $m$ and $n, H_{m, L}$ and $G_{n, L}$ are $\omega_{1}$ saturated ordered divisible Abelian groups. We also observe that by 1.42, $H_{m+1, L} / H_{m, U}$ and $G_{m+1, L} / G_{m, U}$ are $\omega_{1}$-saturated.

We shall construct two families of isomorphisms

$$
\left\{\theta_{n}: G_{n, L} \rightarrow H_{n, L}\right\} \quad \text { and } \quad\left\{\psi_{n}: G_{n, U} \rightarrow H_{n, U}\right\}
$$

such that

(i) $\psi_{n}$ extends $\theta_{n}$.

(ii) $\theta_{n+1}$ extends $\psi_{n}$.

Then, $\theta=\bigcup_{n=1}^{\infty} \theta_{n}$ is the desired isomorphism.

Since $G_{1, L}$ and $H_{1, L}$ are $\omega_{1}$-saturated there exists $\theta_{1}: G_{1, L} \rightarrow$ $H_{1, L}$.

Since $G_{n, U}=R \oplus G_{n, L}$ and $H_{n, U}=R \oplus H_{n, L}$ (each with the lexicographical ordering), we can easily extend $\theta_{n}$ to $\psi_{n}$. It remains to extend $\psi_{n}$ to $\theta_{n+1}$.

Since $G_{n+1, L} / G_{n, U}$ and $H_{n+1, L} / H_{n, U}$ are both $\omega_{1}$-saturated, by 1.41 there exists an isomorphism

$$
\Omega_{n+1}: G_{n+1, L} / G_{n, U} \rightarrow H_{n+1, L} / H_{n, U} .
$$

This yields the following diagram

$$
\begin{aligned}
& 0 \rightarrow G_{n, U} \rightarrow G_{n+1, L} \rightarrow G_{n+1, L} / G_{n, U} \rightarrow 0 \\
& 0 \rightarrow \begin{array}{l}
\psi_{n} \downarrow \\
H_{n, U}
\end{array} \rightarrow H_{n+1, L} \rightarrow H_{n+1, L} / H_{n, U} \rightarrow 0
\end{aligned}
$$


and by the Five-Lemma, there exists an isomorphism

$$
\theta_{n+1}: G_{n+1, L} \rightarrow H_{n+1, L},
$$

making the diagram commute. $\theta_{n+1}$ therefore extends $\psi_{n}$. This proves the lemma.

THEOREM 2.1.17. Let $p$ and $q$ be non-maximal prime ideals of $c$. Let $G_{p}$ denote the valuation group of the valued field $(K=\operatorname{Frac}(c / p)$, $\left.I=c_{0} / p\right)$. Define $G_{q}$ analogously. Let one of the following three conditions hold:

(i) Neither $\left(c_{0} / p\right)_{+}$nor $\left(c_{0} / q\right)_{+}$have countable coinitial subsets.

(ii) Both $\left(c_{0} / p\right)_{+}$and $\left(c_{0} / q\right)_{+}$have countable coinitial subsets of the form $\left\{[f]^{k}\right\}_{k=1}^{\infty}$.

(iii) Both $\left(c_{0} / p\right)_{+}$and $\left(c_{0} / q\right)_{+}$have countable coinitial subsets but neither has a coinitial subset of the form $\left\{[f]^{k}\right\}_{k=1}^{\infty}$

Then $G_{q} \simeq G_{p}$.

Proof. If (i) holds then $G_{q} \simeq G_{p}$ by Proposition 2.1.12 and 1.41.

If (ii) holds, then by Theorem 2.1.15, $G_{q}=R \oplus G_{0, q}$ and $G_{p}=$ $R \oplus G_{0, p}$ with lexicographical ordering and with $G_{0, q}$ and $G_{0, p}$ being $\omega_{1}$-saturated ordered divisible Abelian groups. Then by 1.41, $G_{0, p} \simeq$ $G_{0, q}$. Of course, $\mathbf{R} \simeq \mathbf{R}$, so $G_{q} \simeq G_{p}$.

If (iii) holds, then, by Theorem 2.1.15, $G_{p}=\bigcup_{m=1}^{\infty}\left(\mathbf{R}_{m} \oplus G_{m, p}\right)$ and $G_{q}=\bigcup_{m=1}^{\infty}\left(\mathbf{R}_{m} \oplus G_{m, q}\right)$. By Lemma 2.1.16, these are isomorphic.

What we have done now is classified up to isomorphism all the valuation groups that arise in the valued fields $(K=\operatorname{Frac}(c / p), I=$ $\left.c_{0} / p\right)$. The work of $\mathrm{Ax}$ and Kochen allows us to classify $(c / p)$ by means of these groups and the valuation group of the valued field $(K=$ $\left.\operatorname{Frac}\left(l^{\infty} / c_{0, \mathscr{U}}\right), I=l_{0, \mathscr{U}} / c_{0, \mathscr{U}}\right), \mathscr{U}$ being the ultrafilter associated with $p$. Therefore, we study $l_{0, \mathscr{U}} / c_{0, \mathscr{U}}$.

REMARK 2.1.18. The residue class field of $\left(K=\operatorname{Frac}\left(l^{\infty} / c_{0, \mathscr{U}}\right), I=\right.$ $\left.l_{0, \mathscr{U}} / c_{0, \mathscr{U}}\right)$ is $\mathbf{R}$, so for $\theta$ and $\psi$ in $l^{\infty}$, we have that the following are equivalent:

(i) $v([\theta])>v([\psi])$.

(ii) $r|\psi|>_{\mathscr{U}}|\theta|$ for all $r \in \mathbf{R}^{+}$.

(iii) $r|[\psi]|>|[\theta]|$ for all $r \in \mathbf{R}^{+}$.

(iv) $[\theta] /[\psi] \in l_{0, \mathscr{U}} / c_{0}, \mathscr{U}$.

The following proposition is closely analogous to Proposition 2.1.12. 
Proposition 2.1.19. Let $\mathscr{U}$ be a non-principal ultrafilter on $\mathbf{N}$. Let $\left\{\left[\theta_{j}\right]\right\}$ and $\left\{\left[\psi_{k}\right]\right\}$ be two countable subsets of $\left(l_{0, \mathscr{U}} / c_{0}, \mathscr{U}\right)_{+}$such that $r\left[\psi_{k}\right]>\left[\theta_{j}\right]$ for all $j$ and $k$, and all $r \in \mathbf{R}^{+}$. Let $\left\{\left[\theta_{j}\right]\right\}$ be nonempty. Then there exists $[\Omega] \in l_{0, \mathscr{U}} / \mathcal{c}_{0, \mathscr{U}}$ such that

$$
r\left[\psi_{k}\right]>[\Omega] \text { for all } r \in \mathbf{R}^{+} \text {and all }\left[\psi_{k}\right]
$$

and

$$
r[\Omega]>\left[\theta_{j}\right] \text { for all } r \in \mathbf{R}^{+} \text {and all }\left[\theta_{j}\right]
$$

Proof. We consider 3 cases.

Case 1. $\left\{\left[\psi_{k}\right]\right\}$ is empty. In this case the result follows from Lemma 2.1.9.

Case 2. For each $\left[\theta_{j}\right]$ there exists $\left[\theta_{m}\right]$ such that $r \cdot\left[\theta_{m}\right]>\left[\theta_{j}\right]$ for all $r \in \mathbf{R}^{+}$, and for each $\left[\psi_{k}\right]$ there exists $\left[\psi_{i}\right]$ such that $r \cdot\left[\psi_{k}\right]>\left[\psi_{i}\right]$ for all $r \in \mathbf{R}^{+}$. Then by Lemma 2.1.11, there exists $\Omega \in l^{\infty}$ such that

$$
\psi_{k} \underset{\mathscr{U}}{>} \Omega_{\mathscr{U}}^{>} \theta_{j} \quad \text { for all } k \text { and } j .
$$

Case 3. Either there exists $\left[\theta_{m}\right]$ such that for each $\left[\theta_{j}\right]$ there exists $r \in \mathbf{R}^{+}$such that

$$
\left[\theta_{m}\right]>r\left[\theta_{j}\right]
$$

or there exists $\left[\psi_{i}\right]$ such that for each $\left[\psi_{k}\right]$ there exists $r \in \mathbf{R}^{+}$such that

$$
\left[\psi_{k}\right]>r\left[\psi_{i}\right]
$$

or both.

In this case, we either define a family $\left[\theta_{j}^{\prime}\right]$ or $\left[\psi_{k}^{\prime}\right]$ or both: $\left[\theta_{j}^{\prime}\right]=$ $2^{j}\left[\theta_{m}\right],\left[\psi_{k}^{\prime}\right]=2^{-k}\left[\psi_{i}\right]$. Then the result follows from Lemma 2.1.11.

We did not deal with the case where $\left\{\left[\theta_{j}\right]\right\}$ is empty; that is, we did not show that $\left(l_{0, \mathscr{U}} / c_{0, \mathscr{U}}\right)_{+}$does not have a countable coinitial subset. It turns out that $\left(l_{0, \mathscr{U}} / c_{0}, \mathscr{U}\right)_{+}$can have a countable coinitial subset; in fact, $\left(l_{0, \mathscr{U}} / c_{0, \mathscr{U}}\right)_{+}$can be empty. But there is one kind of coinitial subset that $\left(l_{0, \mathscr{U}} / c_{0, \mathscr{U}}\right)_{+}$cannot have.

Proposition 2.1.20. Let $\mathscr{U}$ be a non-principal ultrafilter on $\mathbf{N}$. Then $\left(l_{0, \mathscr{U}} / c_{0, \mathscr{U}}\right)_{+}$does not have a coinitial subset of the form $\left\{[\theta]^{k}\right\}$.

Proof. Given $\theta \in I_{0, \mathscr{U}}$, with $\theta>_{\mathscr{U}} 0$, we define $\psi$

$$
\psi(n)= \begin{cases}0 & \text { if } \theta(n)=0 \\ e^{-1 /|\theta(n)|} & \text { otherwise. }\end{cases}
$$


Then

$$
\theta^{k} \underset{\mathscr{U}}{>} \psi \underset{\mathscr{U}}{>} f \text { for all } k \text { and for all } f \in c_{0} .
$$

We can now classify, up to isomorphism, the possible valuation groups of the valued fields $\left(K=\left(l^{\infty} / c_{0}, \mathscr{U}\right), I=l_{0, \mathscr{U}} / c_{0, \mathscr{U}}\right)$. In 2.2, we shall show that this classifies $\operatorname{Frac}\left(l^{\infty} / c_{0}, \mathscr{U}\right)$ up to isomorphism. $\operatorname{Frac}\left(l^{\infty} / c_{0}, \mathscr{U}\right)$ is the residue class field of the valued field $(K=$ $\left.\operatorname{Frac}(c / p), I=c_{0} / p\right)$, with $\mathscr{U}$ being the ultrafilter associated with $p$.

THEOREM 2.1.21. Let $\mathscr{U}$ and $\mathscr{V}$ be two non-principal ultrafilters on $\mathbf{N}$. Let $G_{\mathscr{U}}$ be the valuation group of the valued field $(K=$ $\left.\operatorname{Frac}\left(l^{\infty} / c_{0, \mathscr{U}}\right), I=l_{0, \mathscr{U}} / c_{0, \mathscr{U}}\right)$. Define $G_{\mathscr{V}}$ analogously. Let one of the following conditions hold:

(i) $l_{0, \mathscr{U}}=c_{0, \mathscr{U}}$ and $l_{0, \mathscr{V}}=c_{0, \mathscr{V}}$.

(ii) $\left(l_{0, \mathscr{U}} / c_{0, \mathscr{U}}\right)_{+} \neq \varnothing$ and has no countable coinitial subset; and $\left(l_{0, \mathscr{V}} / c_{0, \mathscr{V}}\right)_{+} \neq \varnothing$ and has no countable coinitial subset.

(iii) Both $\left(l_{0, \mathscr{U}} / c_{0, \mathscr{U}}\right)_{+}$and $\left(l_{0, \mathscr{V}} / c_{0, \mathscr{V}}\right)_{+}$have non-empty countable coinitial subsets.

Then $G_{\mathscr{U}} \simeq G_{\mathscr{V}}$.

Proof. If (i) holds, then $G_{\mathscr{U}} \simeq\{0\} \simeq G_{\mathscr{V}}$. If (ii) holds then $G_{\mathscr{U}}$ and $G_{\mathscr{V}}$ are $\omega_{1}$-saturated ordered divisible Abelian groups of cardinality $\aleph_{1}$. By 1.41, $G_{\mathscr{U}} \simeq G_{\mathscr{V}}$. If (iii) holds, then $G_{\mathscr{U}} \simeq G_{\mathscr{V}}$ by Lemma 2.1.16.

We summarize:

(a) The valuation group of the valued field $(K=\operatorname{Frac}(c / p), I=$ $\left.c_{0} / p\right)$ is independent of $\mathscr{U}$, the ultrafilter associated with $p$.

(b) The valuation group of the valued field $\left(K=\operatorname{Frac}\left(l^{\infty} / c_{0}, \mathscr{U}\right)\right.$, $\left.I=l_{0, \mathscr{U}} / c_{0, \mathscr{U}}\right)$ is determined by $\mathscr{U}$.

Finally, one of the hypotheses of the Ax-Kochen Theorem is that the valued field must be $\omega$-pseudo-complete. Therefore, we must show:

Proposition 2.1.22. Let $p$ be a non-maximal prime ideal of $c$. Then the valued field $\left(K=\operatorname{Frac}(c / p), I=c_{0} / p\right)$ is $\omega$-pseudo complete. Let $\mathscr{U}$ be a non-principal ultrafilter on $\mathbf{N}$. Then the valued field $\left(K=\operatorname{Frac}\left(l^{\infty} / c_{0}, \mathscr{U}\right), I=l_{0, \mathscr{U}} / c_{0}, \mathscr{U}\right)$ is $\omega$-pseudo-complete.

Proof. This is actually a fairly simple application of Lemma 2.1.11. We shall only prove this proposition for $\left(K=\operatorname{Frac}\left(l^{\infty} / c_{0}, \mathscr{U}\right), I=\right.$ $\left.l_{0, \mathscr{U}} / c_{0, \mathscr{U}}\right)$. 
Let $\left\{\left[\psi_{k}\right]\right\}$ be a countable subset of $\operatorname{Frac}\left(l^{\infty} / c_{0}, \mathscr{U}\right)$ such that

$$
\frac{\left[\psi_{k-1}\right]-\left[\psi_{k+2}\right]}{\left[\psi_{k}\right]-\left[\psi_{k+1}\right]} \in l_{0, \mathscr{U}} / c_{0, \mathscr{U}} \text { for all } k
$$

That is,

$$
v\left(\left[\psi_{k+2}\right]-\left[\psi_{k+1}\right]\right)>v\left(\left[\psi_{k+1}\right]-\left[\psi_{k}\right]\right), \text { for all } k .
$$

We need to show that there exists $[\psi] \in \operatorname{Frac}\left(l^{\infty} / c_{0}, \mathscr{U}\right)$ such that

$$
\frac{[\psi]-\left[\psi_{k+1}\right]}{[\psi]-\left[\psi_{k}\right]} \in l_{0, \mathscr{U}} / c_{0, \mathscr{U}} \text { for all } k
$$

That is,

$$
v\left([\psi]-\left[\psi_{k+1}\right]\right)>v\left([\psi]-\left[\psi_{k}\right]\right) \text { for all } k .
$$

Toward this end, we construct two subsets of $\operatorname{Frac}\left(l^{\infty} / c_{0}, \mathscr{U}\right), S_{U}$ and $S_{L}$. We construct them in the following manner:

(1) If $\left[\psi_{k+1}\right]>\left[\psi_{k}\right]$, then $\left[\psi_{k}\right] \in S_{L}$ and $\left(2\left[\psi_{k+1}\right]-\left[\psi_{k}\right]\right) \in S_{U}$.

(2) If $\left[\psi_{k+1}\right]<\left[\psi_{k}\right]$, then $\left[\psi_{k}\right] \in S_{U}$ and $\left(2\left[\psi_{k+1}\right]-\left[\psi_{k}\right]\right) \in S_{L}$. By Lemma 2.1.11, there exists $[\psi] \in \operatorname{Frac}\left(l^{\infty} / c_{0}, \mathscr{U}\right),[\psi]$ less than every element of $S_{U}$ and greater than every element of $S_{L}$. It is easy to check that $[\psi]$ has the desired properties.

2.2. Real closed valued fields. Here we will be using the Ax-Kochen machinery to show that there are either exactly 7 or exactly 10 residue class domains of $c$. We first must finish showing that the preconditions for the Ax-Kochen machinery are satisfied. This is checked in Proposition 2.2.3. With Proposition 2.2.7 we begin using the work of Ax and Kochen. We cannot use their results directly because we are trying to show, not only that the valued fields are isomorphic, but also that certain subrings are isomorphic. Therefore we will mimic Kochen's proof of Theorem 1 in [14] (this theorem says that, under certain conditions, two valued fields are isomorphic if their valuation groups and their residue class fields are isomorphic), modifying it to ensure that the subrings are isomorphic. We use this result in two separate steps.

We first consider the valued field $\left(K=\operatorname{Frac}\left(l^{\infty} / c_{0}, \mathscr{U}\right), I=\right.$ $\left.l_{0, \mathscr{U}} / c_{0, \mathscr{U}}\right)$ whose residue class field is $\mathbf{R}$. Then we consider the valued field $\left(K=\operatorname{Frac}(c / p), I=c_{0} / p\right)$, whose residue class field is $\operatorname{Frac}\left(l^{\infty} / c_{0, \mathscr{U}}\right)$.

In the first step, we show that for two ultrafilters $\mathscr{U}$ and $\mathscr{V}$ if the valuation groups of $\left(K=\operatorname{Frac}\left(l^{\infty} / c_{0, \mathscr{U}}\right), I=l_{0, \mathscr{U}} / c_{0, \mathscr{U}}\right)$ and $\left(K=\operatorname{Frac}\left(l^{\infty} / c_{0, \mathscr{V}}\right), I=l_{0, \mathscr{V}} / c_{0, \mathscr{V}}\right)$ are isomorphic, then we have 
an isomorphism from $\operatorname{Frac}\left(l^{\infty} / c_{0, \mathscr{U}}\right)$ to $\operatorname{Frac}\left(l^{\infty} / c_{0, \mathscr{V}}\right)$ which sends the constant functions to the constant functions. As we showed in 2.1 there are at most three different valuation groups for $(K=$ $\left.\operatorname{Frac}\left(l^{\infty} / c_{0, \mathscr{U}}\right), I=l_{0, \mathscr{U}} / c_{0, \mathscr{U}}\right)$, including the trivial group $\{0\}$.

In the second step, for two non-maximal prime ideals $p, q \subset c$, with $\mathscr{U}$ and $\mathscr{V}$ as the ultrafilters associated with $p$ and $q$, we show that: If

(a) we have an isomorphism from $\operatorname{Frac}\left(l^{\infty} / c_{0, \mathscr{U}}\right)$ to $\operatorname{Frac}\left(l^{\infty} / c_{0, \mathscr{V}}\right)$ which sends the constant functions to the constant functions and

(b) The valuation groups of $\left(K=\operatorname{Frac}(c / p), I=c_{0} / p\right)$ and $(K=$ $\left.\operatorname{Frac}(c / q), I=c_{0} / q\right)$ are isomorphic.

Then we have an isomorphism from $\operatorname{Frac}(c / p)$ to $\operatorname{Frac}(c / q)$, such that the image of $c / p$ is $c / q$.

As we showed in 2.1, there are three possible valuation groups for $\left(K=\operatorname{Frac}(c / p), I=c_{0} / p\right)$. The three choices for valuation group of $\left(K=\operatorname{Frac}(c / p), I=c_{0} / p\right)$ and at most three choices for the valuation group of $\left(K=\operatorname{Frac}\left(l^{\infty} / c_{0}, \mathscr{U}\right), I=l_{0, \mathscr{U}} / c_{0, \mathscr{U}}\right)$ yield at most 9 residue class domains $c / p$, with $p$ non-maximal.

DEFINITION 2.2.1. A real closed valued field with convex valuation is a valued field $(K, R, I, G, v, \bar{K})$ such that

(i) $K$ is a real closed field.

(ii) For $a, b \in K$, if $a>b>0$ then $v(b) \geq v(a)$.

We first prove some simple but essential results about real closed fields with convex valuation which will enable us to use the Ax-Kochen machinery.

LEMma 2.2.2. For a valued field $(K, R, I, G, v, \bar{K})$, the following are equivalent:

(i) $(K, R, I, G, v, \bar{K})$ is a real closed valued field with convex valuation.

(ii) $K$ is a real closed field and $I$ is a convex subset of $K$.

(iii) $R$ is a real closed ring.

Proof. Left to reader.

Proposition 2.2.3. Let $(K, R, I, G, v, \bar{K})$ be a real closed field with convex valuation. Then

(i) $(K, R, I, G, v, \bar{K})$ is a Hensel field. 
(ii) $\bar{K}$ is a real closed field.

(iii) $K^{\#} /\{-1,1\}$ is a divisible group.

(iv) $G$ is a divisible group.

(v) There exists a cross-section $\pi: G \rightarrow K^{\#}$.

Proof. (i) Let $g(x) \in R[x]$ and let $\bar{g}(x)=f(x) \in \bar{K}[x]$. If $f$ has a non-singular root at $\alpha$, then $f$ changes sign at $\alpha$. Let $\beta, \gamma \in \bar{K}$ be such that (w.l.o.g.)

(1) $\beta<\alpha<\gamma$,

(2) for $\beta<y<\alpha$ we have $f(y)>0$, and

(3) for $\alpha<y<\gamma$ we have $f(y)<0$.

Then, for $z \in R$, if $\beta<\bar{z}<\alpha$ we have $g(z)>0$, and for $\alpha<\bar{z}<\gamma$ we have $g(z)<0$. Since $g$ changes sign and $R$ is real closed, there exists $a \in R$ such that $g(a)=0$ and $\bar{a}=\alpha$.

(ii) Clearly, every positive element of $\bar{K}$ has a square root. So let $f(x) \in \bar{K}[x]$ be an odd degree polynomial. Clearly there exists $g(x) \in R[x]$ such that

(1) $\operatorname{deg} g(x)=\operatorname{deg} f(x)$, which is odd,

(2) $\bar{g}(x)=f(x)$, and

(3) the leading coefficient of $g(x)$ is a unit of $R$. Since $R$ is real closed, there exists $b \in R$ such that $g(b)=0$. Then $f(\bar{b})=0$.

(iii) For all positive $b$, for all $n$, there exists $c \in K$ such that $c^{n}=b$.

(iv) The image of a divisible Abelian group is always divisible.

(v) Torsion-free divisible Abelian groups are vector spaces over $\mathbf{Q}$. Hence all exact sequences split.

COROllary 2.2.4. Let $p$ be a non-maximal prime ideal of $c$. Let $(K, R, I, G, v, \bar{K})$ be the valued field $\left(K=\operatorname{Frac}(c / p), I=c_{0} / p\right)$. Then $\bar{K}$ is a real closed field, $G$ is a divisible group, and there is $a$ cross-section $\pi: G \rightarrow K^{\#}$. Further, $\left(K=\operatorname{Frac}(c / p), I=c_{0} / p\right)$ is $a$ Hensel field.

COROLlaRY 2.2.5. Let $\mathscr{U}$ be a non-principal ultrafilter on $\mathbf{N}$. Then $\operatorname{Frac}\left(l^{\infty} / c_{0, \mathscr{U}}\right)$ is a real closed field, and $l^{\infty} / c_{0, \mathscr{U}}$ is a real closed ring.

We now begin exploiting the work of Ax and Kochen.

Notation 2.2.6. Let $(K, R, I, G, v, \bar{K})$ and $\left(K^{\prime}, R^{\prime}, I^{\prime}, G^{\prime}\right.$, $\left.v^{\prime}, \bar{K}^{\prime}\right)$ be two valued fields. Let $E \subset K$ and $E^{\prime} \subset K^{\prime}$ be subfields. 
Let

$\left(\theta, \theta^{\#}\right): \quad\left(\left(E, R \cap E, I \cap E, v(E),\left.v\right|_{E}, \bar{E}\right),\left.\pi\right|_{v(E)}\right)$

$\rightarrow\left(\left(E^{\prime}, R^{\prime} \cap E^{\prime}, I^{\prime} \cap E^{\prime}, v^{\prime}\left(E^{\prime}\right),\left.v^{\prime}\right|_{E^{\prime}}, \bar{E}^{\prime}\right),\left.\pi^{\prime}\right|_{v^{\prime}\left(E^{\prime}\right)}\right)$

be a pure map. We hereafter simply denote this as

$$
\theta: E \rightarrow E^{\prime}
$$

when there is no danger of confusion.

We have the following important fact from [14].

Proposition 2.2.7. Let $((K, R, I, G, v, \bar{K}), \pi)$ and $\left(\left(K^{\prime}, R^{\prime}, I^{\prime}\right.\right.$, $\left.\left.G^{\prime}, v^{\prime}, \bar{K}^{\prime}\right), \pi^{\prime}\right)$ be $\omega$-pseudo-complete Hensel fields with cross-section such that

(i) $\operatorname{Card} K=\operatorname{Card} K^{\prime}=\aleph_{1}$.

(ii) $\operatorname{char} \bar{K}=\operatorname{char} \bar{K}^{\prime}=0$.

(iii) There exists an isomorphism $\psi^{\#}: G \rightarrow G^{\prime}$.

(iv) $\bar{K} \simeq \bar{K}^{\prime}$.

Let $E \subset K$ and $E^{\prime} \subset K^{\prime}$ be subfields such that $\bar{E}=\bar{K}$ and $\bar{E}^{\prime}=\bar{K}^{\prime}$. Let $\theta: E \rightarrow E^{\prime}$ be a pure map such that $\theta^{\#}: v(E) \rightarrow v^{\prime}\left(E^{\prime}\right)$ is the restriction of $\psi^{\#}$ to $v(E)$. Let $c \in K$. Then there exists a subfield $F \subset K$, with $c \in F, E \subset F$, and a pure map $\rho: F \rightarrow F^{\prime}$ such that $\rho$ extends $\theta$ ( $\rho^{\#}$ thus extends $\left.\theta^{\#}\right)$, and $\rho^{\#}$ is the restriction of $\psi^{\#}$ to $v(F)$.

\section{Proof. ([14], Proposition 3.)}

Combining Proposition 2.2.3 and Proposition 2.2.7 we have:

Proposition 2.2.8. Let $(K, R, I, G, v, \bar{K})$ and $\left(K^{\prime}, R^{\prime}, I^{\prime}, G^{\prime}\right.$, $\left.v^{\prime}, \bar{K}^{\prime}\right)$ be $\omega$-pseudo-complete real closed valued fields with convex valuation. Let $\pi$ and $\pi^{\prime}$ be the cross-sections guaranteed by Proposition 2.2.3. Let

(i) $\operatorname{Card} K=\operatorname{Card} K^{\prime}=\aleph_{1}$,

(ii) $\psi^{\#}: G \rightarrow G^{\prime}$ be an isomorphism,

(iii) $\bar{K} \simeq \bar{K}^{\prime}$.

Let $E \subset K$ and $E^{\prime} \subset K^{\prime}$ be subfields such that $\bar{E}=\bar{K}$ and $\bar{E}^{\prime}=\bar{K}^{\prime}$. Let $\theta: E \rightarrow E^{\prime}$ be a pure map such that $\theta^{\#}: v(E) \rightarrow v^{\prime}\left(E^{\prime}\right)$ is the restriction of $\psi^{\#}$ to $v(E)$. Let $c \in K$. Then there exists a subfield $F \subset K$, with $c \in F$ and $E \subset F$, and a pure map $\rho: F \rightarrow F^{\prime}$ such 
that $\rho$ extends $\theta$ (thus $\rho^{\#}$ extends $\left.\theta^{\#}\right)$, and $\rho^{\#}$ is the restriction of $\psi^{\#}$ to $v(F)$.

From Proposition 2.2.8, we can see that once we get the first such pure map between such subfields of $K$ and $K^{\prime}$, we can extend it by induction to a cross-analytic isomorphism between the two valued fields with cross section. We do not want the cross-analytic isomorphism for its own sake, of course. We want at last to be able, under certain conditions, to construct an isomorphism between $c / p$ and $c / q$. To do this, we use the fact that in the valued field $\left(K=\operatorname{Frac}(c / p), I=c_{0} / p\right)$, $c_{0} / p=I \subset c / p$. Also, in the valued field $\left(K=\operatorname{Frac}\left(l^{\infty} / c_{0}, \mathscr{U}\right), I=\right.$ $\left.l_{0, \mathscr{U}} / c_{0, \mathscr{U}}\right)$, the valuation ideal $l_{0, \mathscr{U}} / c_{0, \mathscr{U}}$ has trivial intersection with the image of $c / p$.

Proposition 2.2.9. Let $\mathscr{U}$ and $\mathscr{V}$ be non-principal ultrafilters on $\mathbf{N}$. Let one of the following conditions hold:

(1) $\left(l^{\infty} / c_{0, \mathscr{U}}\right) \simeq \mathbf{R}$ and $\left(l^{\infty} / c_{0, \mathscr{V}}\right) \simeq \mathbf{R}$.

(2) $\left(l_{0, \mathscr{U}} / c_{0, \mathscr{U}}\right)_{+}$has no countable coinitial subset and $\left(l_{0, \mathscr{V}} / c_{0, \mathscr{V}}\right)_{+}$ has no countable coinitial subset.

(3) $\left(l_{0, \mathscr{U}} / c_{0, \mathscr{U}}\right)_{+}$has a non-empty countable coinitial subset; so does $\left(l_{0, \mathscr{V}} / c_{0, \mathscr{V}}\right)_{+}$.

Then there exists an isomorphism

$$
\theta: l^{\infty} / c_{0, \mathscr{U}} \rightarrow l^{\infty} / c_{0, \mathscr{V}}
$$

such that for $f \in c$,

$$
\theta\left(\left[f+c_{0, \mathscr{U}}\right]\right)=\left[f+c_{0, \mathscr{V}}\right] .
$$

Proof. We first observe that the subsets

$$
E_{1}=\left\{\left[f+c_{0, \mathscr{U}}\right] \mid f \in c\right\} \subset l^{\infty} / \mathcal{c}_{0}, \mathscr{U}
$$

and

$$
E_{1}^{\prime}=\left\{\left[f+c_{0, \mathscr{V}}\right] \mid f \in c\right\} \subset l^{\infty} / c_{0, \mathscr{V}}
$$

are both isomorphic to $\mathbf{R}$. We have isomorphisms $\chi_{\mathscr{U}}$ and $\chi_{\mathscr{V}}$ such that

$$
\begin{aligned}
& \chi_{\mathscr{U}}\left(\left[f+c_{0, \mathscr{U}}\right]\right)=\lim _{\mathscr{U}} f=\lim _{n \rightarrow \infty} f(n), \\
& \chi_{\mathscr{V}}\left(\left[f+c_{0, \mathscr{V}}\right]\right)=\lim _{\mathscr{V}} f=\lim _{n \rightarrow \infty} f(n) .
\end{aligned}
$$

So we have

$$
\theta_{1}: E_{1} \rightarrow E_{1}^{\prime} \quad \text { with } \theta_{1}=\chi_{\mathscr{V}}^{-1} \circ \chi_{\mathscr{U}}
$$


This, of course, disposes of case (1).

For the other two cases, since $v$ is trivial on $E_{1}$ and $v^{\prime}$ is trivial on $E_{1}^{\prime}, \theta_{1}$ is trivially a pure map.

We extend $\theta_{1}$ by induction using Proposition 2.2.8. It is therefore necessary to show that the valued fields $\left(K=\operatorname{Frac}\left(l^{\infty} / c_{0}, \mathscr{U}\right), I=\right.$ $\left.l_{0, \mathscr{U}} / c_{0, \mathscr{U}}\right)$ and $\left(K=\operatorname{Frac}\left(l^{\infty} / \mathcal{c}_{0, \mathscr{V}}\right), I=l_{0, \mathscr{V}} / c_{0, \mathscr{V}}\right)$ satisfy the hypotheses of Proposition 2.2.8.

By Lemma 2.2.2 and Corollary 2.2.5, these are both real closed fields with convex valuations and in both cases the residue class field is $\mathbf{R}$; hence the residue class fields are isomorphic to each other. In fact, $\theta_{1}$ induces this isomorphism. By 2.1.21, the valuation groups are isomorphic (and $\theta_{1}^{\#}:\{0\} \rightarrow\{0\}$ is certainly the restriction of this isomorphism). By 2.1.22, both valued fields are $\omega$-pseudo-complete. Clearly, they each have cardinality $\aleph_{1}=2^{\aleph_{0}}$. The hypotheses of Proposition 2.2.8, then, are satisfied.

So we well order the elements of $\operatorname{Frac}\left(l^{\infty} / \mathcal{c}_{0, \mathscr{U}}\right),\left\{x_{\alpha}\right\}_{\alpha<\omega_{1}}$, and the elements of $\operatorname{Frac}\left(l^{\infty} / c_{0, \mathscr{V}}\right),\left\{y_{\alpha}\right\}_{\alpha<\omega_{1}}$. We shall show that there exists a family of pure maps $\left\{\theta_{\alpha}: E_{\alpha} \rightarrow E_{\alpha}^{\prime}\right\}_{\alpha<\omega_{1}}$ such that

(a) $\bigcup_{\alpha<\omega_{1}} E_{\alpha}=\operatorname{Frac}\left(l^{\infty} / c_{0, \mathscr{U}}\right)$.

(b) $\bigcup_{\alpha<\omega_{1}} E_{\alpha}^{\prime}=\operatorname{Frac}\left(l^{\infty} / c_{0, \mathscr{V}}\right)$.

(c) $\theta=\bigcup_{\alpha<\omega_{1}} \theta_{\alpha}$ is the desired isomorphism.

We have shown above that $\theta_{1}: E_{1} \rightarrow E_{1}^{\prime}$ exists, and that $\bar{E}_{1}=\bar{K} \simeq$ $\bar{K}^{\prime}=\bar{E}_{1}$.

Let $\alpha=\lambda+2 n$ where $\lambda$ is a limit ordinal and $n$ is a non-negative integer. Given $\theta_{\alpha}: E_{\alpha} \rightarrow E_{\alpha}^{\prime}$, by Proposition 2.2.7 there exists $\theta_{\alpha+1}$ : $E_{\alpha+1} \rightarrow E_{\alpha+1}^{\prime}$ such that: $\theta_{\alpha+1}$ extends $\theta_{\alpha}, E_{\alpha} \subset E_{\alpha+1}, E_{\alpha}^{\prime} \subset E_{\alpha+1}^{\prime}$, and $x_{(\lambda+n)} \in E_{\alpha+1}$.

Let $\alpha=\lambda+2 n+1$. Given $\theta_{\alpha}: E_{\alpha} \rightarrow E_{\alpha}^{\prime}$, there trivially exists $\theta_{\alpha}^{-1}: E_{\alpha}^{\prime} \rightarrow E_{\alpha}$ and by Proposition 2.2.7 there exists $\theta_{\alpha+1}^{-1}: E_{\alpha+1}^{\prime} \rightarrow$ $E_{\alpha+1}$ where $\theta_{\alpha+1}^{-1}$ extends $\theta_{\alpha}^{-1}$ and $y_{\lambda+n} \in E_{\alpha+1}^{\prime}$.

Finally, for limit ordinals $\lambda$,

$$
\theta_{\lambda}=\bigcup_{\alpha<\lambda} \theta_{\alpha}, \quad E_{\lambda}=\bigcup_{\alpha<\lambda} E_{\alpha} \quad \text { and } \quad E_{\lambda}^{\prime}=\bigcup_{\alpha<\lambda} E_{\alpha}^{\prime} .
$$

This completes the proof.

We shall show later, in $\S 3$, that all 3 cases of Proposition 2.2.9, (1), (2), and (3), occur.

We wish to prove a proposition similar to the preceding one for the valued field $\left(K=\operatorname{Frac}(c / p), I=c_{0} / p\right)$. However, since we do not have an obvious image of the residue class field in the main field, as 
the constant functions are an obvious image of $\mathbf{R}$, it is not quite as easy to get the initial pure map. Toward that end, we have:

LEMMA 2.2.10. Let $(K, R, I, G, v, \bar{K})$ be a real closed valued field with convex valuation. Let $K_{0}$ be a maximal subfield of $K$ on which the valuation is trivial. Then

$$
K_{0} \simeq \bar{K}_{0}=\bar{K} .
$$

Proof. Clearly $K_{0} \simeq \bar{K}_{0} \subset \bar{K}$. Assume towards a contradiction that $\beta \in \bar{K}-\bar{K}_{0}$. Let $b \in K$ be such that $\bar{b}=\beta$. Consider two cases:

Case 1. For all $P \in K_{0}[x]$, (except $\left.P=0\right), v(P(b))=0$.

Then $K_{0}(b)$ is an extension of $K_{0}$ on which the valuation is trivial, contrary to assumption.

Case 2. There exists $P \in K_{0}[x], P \neq 0$, such that $v(P(b))>0$.

The set of such $P$ (including 0 ) forms an ideal of $K_{0}[x]$, and since $K_{0}[x]$ is a PID we let $P_{1}$ be the generator of this ideal. We then have $v\left(P_{1}(b)\right)>0$, and hence $\bar{P}_{1}(\beta)=0$; and by Henselity (Proposition 2.2.3), there exists $c \in K$ such that $\bar{c}=\beta$ and $P_{1}(c)=$ 0 . $K_{0}(c)$ is then an extension of $K_{0}$ on which the valuation is trivial, contradicting the hypothesis.

Lemma 2.2.11. Let $(K, R, I, G, v, \bar{K})$ and $\left(K^{\prime}, R^{\prime}, I^{\prime}, G^{\prime}, v^{\prime}\right.$, $\bar{K}^{\prime}$ ) be real closed valued fields with convex valuation. Let $\bar{K} \simeq \bar{K}^{\prime}$. Then there exist subfields $K_{0} \subset K$ and $K_{0}^{\prime} \subset K^{\prime}$ such that

(a) $K_{0} \simeq \bar{K}_{0}=\bar{K} \simeq \bar{K}^{\prime}=\bar{K}_{0}^{\prime} \simeq K_{0}^{\prime}$.

(b) $v$ is trivial on $K_{0}$.

(c) $v^{\prime}$ is trivial on $K_{0}^{\prime}$.

Proof. This follows immediately from Lemma 2.2.10.

Proposition 2.2.12. Let $p$ and $q$ be non-maximal prime ideals of $c$. Let $\mathscr{U}$ be the ultrafilter associated with $p$ and let $\mathscr{V}$ be the ultrafilter associated with $q$. Let there exist an isomorphism

$$
\theta:\left(l^{\infty} / c_{0, \mathscr{U}}\right) \rightarrow\left(l^{\infty} / c_{0, \mathscr{V}}\right)
$$

such that for $f \in c$,

$$
\theta\left(\left[f+c_{0, \mathscr{U}}\right]\right)=\left[f+c_{0, \mathscr{V}}\right],
$$

and let one of the following conditions hold:

(a) Neither $\left(c_{0} / p\right)_{+}$nor $\left(c_{0} / q\right)_{+}$has a countable coinitial subset. 
(b) Both $\left(c_{0} / p\right)_{+}$and $\left(c_{0} / q\right)_{+}$have a countable coinitial subset of the form $\left\{[f]^{k}\right\}_{k=1}^{\infty}$.

(c) Both $\left(c_{0} / p\right)_{+}$and $\left(c_{0} / q\right)_{+}$have countable coinitial subsets, but neither has a coinitial subset as in (b).

Then $c / p \simeq c / q$.

Proof. We will proceed on lines similar to those in the proof of Proposition 2.2.9. We shall construct a cross-analytic isomorphism between the valued fields $\left(K=\operatorname{Frac}(c / p), I=c_{0} / p\right)$ and $(K=$ $\left.\operatorname{Frac}(c / q), I=c_{0} / q\right)$. This will certainly map $c_{0} / p$ one to one onto $c_{0} / q$. We shall also ensure that for a constant sequence $r$, the image of $[r+p]$ is $[r+q]$.

By Lemma 2.2.2, $\left(K=\operatorname{Frac}(c / p), I=c_{0} / p\right)$ and $(K=\operatorname{Frac}(c / q)$, $\left.I=c_{0} / q\right)$ are real closed valued fields with convex valuation. By 2.1.22, they are $\omega$-pseudo-complete. By 2.1.17, the valuation groups are isomorphic. Clearly $\operatorname{Card}(\operatorname{Frac}(c / p))=\operatorname{Card}(\operatorname{Frac}(c / q))=\aleph_{1}=$ $2^{\aleph_{0}}$.

We recall that

$$
\operatorname{Frac}(c / p) \simeq \operatorname{Frac}\left(l^{\infty} / p_{b}\right) \text { and } \operatorname{Frac}(c / q) \simeq \operatorname{Frac}\left(l^{\infty} / q_{b}\right),
$$

and that the image of $c_{0} / p$ and $c_{0} / q$ in $\operatorname{Frac}\left(l / p_{b}\right)$ and $\operatorname{Frac}\left(l^{\infty} / q_{b}\right)$ are $c_{0, \mathscr{U}} / p_{b}$ and $c_{0, \mathscr{V}} / q_{b}$. Clearly the residue class field of $(K=$ $\left.\operatorname{Frac}(c / p), I=c_{0} / p\right)$ is isomorphic to $\operatorname{Frac}\left(l^{\infty} / c_{0}, \mathscr{U}\right)$ and the residue class field of $\left(K=\operatorname{Frac}(c / q), I=c_{0} / q\right)$ is isomorphic to $\operatorname{Frac}\left(l^{\infty} / c_{0, \mathscr{V}}\right)$.

We let $K_{0}$ be a subfield of $\operatorname{Frac}(c / p)$ and $K_{0}^{\prime}$ be a subfield of $\operatorname{Frac}(c / q)$ such that

(i) For any constant sequence $r$,

$$
[r+p] \in K_{0} \quad \text { and } \quad[r+q] \in K_{0}^{\prime} .
$$

(ii) $v$ is trivial on $K_{0}$ and $v^{\prime}$ is trivial on $K_{0}^{\prime}$.

(iii) $K_{0}$ and $K_{0}^{\prime}$ are maximal with respect to property (ii).

Next by Lemma 2.2.10, we have isomorphisms

$$
\chi_{p}: K_{0} \rightarrow \operatorname{Frac}\left(l^{\infty} / c_{0}, \mathscr{U}\right)
$$

and

$$
\chi_{q}: K_{0}^{\prime} \rightarrow \operatorname{Frac}\left(l^{\infty} / c_{0, \mathscr{V}}\right)
$$

such that for any constant sequence $r$,

$$
\begin{aligned}
& \chi_{p}([r+p])=\left[r+c_{0, \mathscr{U}}\right], \\
& \chi_{q}([r+q])=\left[r+c_{0, \mathscr{V}}\right] .
\end{aligned}
$$


Then

$$
\psi_{1}=\chi_{q}^{-1} \circ \theta \circ \chi_{p}
$$

is our desired initial pure map.

The proof can be completed by transfinite induction as in Proposition 2.2.9.

Together Propositions 2.2.9 and 2.2.12 give us the following, which is the main theorem of this section.

THEOREM 2.2.13. Let $p$ and $q$ be non-maximal prime ideals of $c$. Let $\mathscr{U}$ be the ultrafilter associated with $p$. Let $\mathscr{V}$ be the ultrafilter associated with $q$. Let one of the conditions (1) to (3) below hold, and let one of conditions (a) to (c) below hold.

(1) $l^{\infty} / c_{0, \mathscr{U}} \simeq \mathbf{R}$ and $l^{\infty} / c_{0, \mathscr{V}} \simeq \mathbf{R}$.

(2) $\left(l_{0, \mathscr{U}} / \mathcal{c}_{0, \mathscr{U}}\right)_{+}$has no countable coinitial subset; neither does $\left(l_{0, \mathscr{V}} / c_{0, \mathscr{V}}\right)_{+} \cdot$

(3) $\left(l_{0, \mathscr{U}} / c_{0}, \mathscr{U}\right)_{+}$has a non-empty countable coinitial subset, so does $\left(l_{0, \mathscr{V}} / c_{0, \mathscr{V}}\right)_{+}$.

(a) Neither $\left(c_{0} / p\right)_{+}$nor $\left(c_{0} / q\right)_{+}$has a countable coinitial subset.

(b) Both $\left(c_{0} / p\right)_{+}$and $\left(c_{0} / q\right)_{+}$have coinitial subsets of the form $\left\{[f]^{k}\right\}_{k \in \mathbf{N}}$.

(c) Both $\left(c_{0} / p\right)_{+}$and $\left(c_{0} / q\right)_{+}$have countable coinitial subsets but not of the form $\left\{[f]^{k}\right\}_{k \in \mathbf{N}}$.

Then $c / p \simeq c / q$.

THEOREM 2.2.14. There are at most 10 residue class domains of $c$.

Proof. Let $p$ be a maximal ideal of $c$, then $c / p \simeq \mathbf{R}$. For $p$ nonmaximal, there are at most the 9 cases listed in Theorem 2.2.13.

We shall show later that there are exactly 10 residue class domains of $c$.

3. Ultrafilters and $\left(l_{0, \mathscr{U}} / c_{0, \mathscr{U}}\right)$. In this section we show that there are exactly 10 residue class domains of $c$. Toward that end, we show that all three cases of 2.2.9 do occur. That is, we show that there exist ultrafilters $\mathscr{U}, \mathscr{V}$, and $\mathscr{W}$ on $\mathbf{N}$ such that

(i) $\left(l^{\infty} / c_{0}, \mathscr{U}\right) \simeq \mathbf{R}$.

(ii) $\left(l_{0}, \mathscr{W} / c_{0}, \mathscr{W}\right)_{+}$has no countable coinitial subset.

(iii) $\left(l_{0}, \mathscr{W} / c_{0}, \mathscr{W}\right)_{+}$has a non-empty countable coinitial subset. 
Satisfying (i) is equivalent to $\mathscr{U}$ being a $P$-point ultrafilter. The existence of $P$-point ultrafilters is guaranteed by Martin's Axiom, a fortiori by the Continuum Hypothesis. On the other hand it is consistent with ZFC that $P$-points do not exist (see [2], [21], [23], and [24]).

Necessary and sufficient conditions for ultrafilters to satisfy (ii) are not known; neither are those for (iii). We show that to satisfy (ii), it is sufficient that $\mathscr{V}$ be a limit point of a discrete sequence of nonprincipal ultrafilters. In 3.2 we construct an ultrafilter $\mathscr{W}$ satisfying (iii), which is obviously neither a $P$-point nor a limit point of a discrete set of non-principal ultrafilters.

It is known (see [23]), that $\mathrm{CH}$ guarantees the existence of ultrafilters that are neither $P$-points nor limit points of discrete sets of non-principal ultrafilters. There are two cases (both guaranteed):

(a) Weak $P$-points that are not limit points of any countable subset of $\beta \mathbf{N}-\mathbf{N}$.

(b) Ultrafilters that are limit points of a countable subset of $\beta \mathbf{N}-\mathbf{N}$, but not of a discrete countable subset.

Interestingly, the residue class domains themselves can distinguish 3 types of non-principal ultrafilters on $\mathbf{N}$, while the first order theories of these domains can only distinguish 2 types [19].

3.1. P-points and limit points of sequences of ultrafilters. We first show that the case $l^{\infty} / c_{0, \mathscr{U}} \simeq \mathbf{R}$ does, in fact, occur.

LEMMA 3.1.1. There exists a non-principal ultrafilter $\mathscr{U}$ on $\mathbf{N}$ such that: Given a sequence of subsets of $\mathbf{N},\left\{A_{j}\right\}_{j=1}^{\infty}$ with

(a) $A_{j+1} \subset A_{j}$,

(b) $A_{j} \in \mathscr{U}$ for all $j$,

there exists $A \in \mathscr{U}$ such that $\left(A-A_{j}\right)$ is finite for all $j$.

Proof. [21].

Ultrafilters $\mathscr{U}$ such as in Lemma 1 are called $P$-point ultrafilters (strictly speaking, they are the $P$-points of $\beta \mathbf{N}-\mathbf{N}$ ).

LEMMA 3.1.2. For $\mathscr{U}$ as in Lemma 1, if $\theta \in l^{\infty}$, then there exists $f \in c$ such that $\theta \equiv_{\mathscr{U}} f$.

Proof. Let $r=\lim _{\mathscr{U}} \theta$. Let $A_{j}=\{n|| \theta(n)-r \mid \leq 1 / j\}$. Clearly, $A_{j+1} \subset A_{j}$ and $A_{j} \in \mathscr{U}$ for all $j$. Hence there exists $A \in \mathscr{U}$ such 
that for all $j$, the set

$$
\{n \in A|| \theta(n)-r \mid>1 / j\}
$$

is finite. Define $f$ :

$$
f(n)= \begin{cases}\theta(n) & \text { for } n \in A, \\ r & \text { for } n \notin A .\end{cases}
$$

Then $f \equiv_{\mathscr{U}} \theta$ and $f \in \mathcal{C}$.

Proposition 3.1.13. For $\mathscr{U}$ as in Lemma 1,

$$
l^{\infty} / c_{0, \mathscr{U}} \simeq \mathbf{R}
$$

Proof. For $\psi, \theta \in l^{\infty}$, let $\lim _{\mathscr{U}} \theta=\lim _{\mathscr{U}} \psi$. Then $\lim _{\mathscr{U}}(\psi-\theta)=$ 0 . By Lemma 3.1.2, there exists $f \in c$ such that $f \equiv_{\mathscr{U}} \psi-\theta$. Clearly, $\lim _{n \rightarrow \infty} f(n)=0$, so $(\psi-\theta) \in c_{0, \mathscr{U}}$. This proves the proposition.

The preceding proposition was first proved in [8], Th. 3.2.2 by Cherlin, Dickmann, and Louveau.

We next show that the case does occur where $\left(l_{0, \mathscr{V}} / c_{0, \mathscr{V}}\right)_{+}$is nonempty and has no countable coinitial subset.

LEMMA 3.1.4. Let $\left\{\mathscr{U}_{j}\right\}_{j=1}^{\infty}$ be a countable family of non-principal ultrafilters on $\mathbf{N}$. Let there exist a family of subsets of $\mathbf{N},\left\{A_{j}\right\}_{j=1}^{\infty}$ such that

(i) $A_{j} \in \mathscr{U}_{j}$,

(ii) $A_{j} \cap A_{i}=\varnothing$ for $i \neq j$.

Let $\mathscr{U}$ be a non-principal ultrafilter on $\mathbf{N}$. Then the family of subsets $\mathscr{V}$, defined by

$$
\mathscr{V}=\left\{B \subset \mathbf{N} \mid\left\{j \mid B \in \mathscr{U}_{j}\right\} \in \mathscr{U}\right\}
$$

is an ultrafilter.

Proof. Left to reader.

Proposition 3.1.5. Let $\mathscr{V}$ be an ultrafilter as in Lemma 4. Then $\left(l_{0, \mathscr{V}} / c_{0, \mathscr{V}}\right)_{+}$has no countable coinitial subset.

Proof. Assume toward a contradiction that $\left\{\left[\theta_{k}\right]\right\}$ is a countable coinitial subset of $\left(l_{0, \mathscr{V}} / c_{0, \mathscr{V}}\right)_{+}$. Choose representatives of each congruence class, $\psi_{k} \in\left[\theta_{k}\right]$, such that $\psi_{k}(n) \geq \psi_{n+1}(n)>0$ for all $k$ and $n$. This then gives us that for any ultrafilter $\mathscr{U}_{j}$, if

$$
\psi_{k} \underset{\mathscr{U}_{1}}{>}|f| \text { for all } f \in c_{0}
$$


and if $i<k$, then

$$
\psi_{i} \underset{\mathscr{U}_{1}}{>}|f| \text { for all } f \in c_{0} .
$$

We define $\theta \in l^{\infty}$ on $A_{j}$ by: $\theta(n)=\psi_{k}(n)$, where

$$
k=\max \left\{1, \sup \left\{m \leq j\left|\psi_{m} \underset{\mathscr{U}_{j}}{>}\right| f \mid \text { for all } f \in c_{0}\right\}\right\} .
$$

Clearly, then,

$$
\left\{j\left|\psi_{1} \overrightarrow{\mathcal{U}}_{,}\right| f \mid \text { for all } f \in c_{0}\right\}=\left\{j\left|\theta_{\vec{U}_{1}}\right| f \mid \text { for all } f \in c_{0}\right\}
$$

and for all $m$

$$
\left\{j\left|\psi_{m} \underset{\mathscr{U}_{J}}{>_{j}}\right| f \mid \text { for all } f \in c_{0}\right\}=\left\{j \geq m \mid \psi_{m}{\underset{\mathscr{U}}{\rho}}_{j} \theta\right\} \text {. }
$$

Hence, for all $m$, and for all $f \in c_{0}$,

$$
\psi_{m} \underset{\mathscr{V}}{>} \theta \underset{\mathscr{V}}{>}|f|
$$

contrary to assumption.

3.2. Ultrafilters $\mathscr{W}$ such that $\left(l_{0}, \mathscr{W} / c_{0}, \mathscr{W}\right)_{+}$has a countable coinitial subset. Finally we wish to show that there exists a non-principal ultrafilter $\mathscr{W}$ such that $\left(l_{0, \mathscr{W}} / \mathcal{c}_{0}, \mathscr{W}\right)_{+}$has a (non-empty) countable coinitial subset. This amounts to the following: We need to construct an ultrafilter $\mathscr{W}$ containing a doubly indexed countable family of subsets of $\mathbf{N}$,

$$
\left\{A_{j, k}\right\}_{j=1 k=1}^{\infty}
$$

where for each $\left[\theta_{j}\right]$ in the countable coinitial subset

$$
A_{j, k}=\theta_{j}^{-1}((0,1 / k]) \text {. }
$$

It is also necessary that for any $j \in \mathbf{N}$, if a set $B_{j}$ is such that $B_{j}-A_{j, k}$ is finite for all $k \in \mathbf{N}$, then $B_{j}^{c} \in \mathscr{W}$; otherwise $\theta_{j}$ would converge $(\bmod \mathscr{W})$. It is further necessary that for any $\psi: \mathbf{N} \rightarrow \mathbf{R}$, such that $\theta_{J}>\mathscr{W}|\psi|$ for all $j \in \mathbf{N}, \psi$ should converge $(\bmod \mathscr{W})$.

If any countable coinitial subset exists then there exists a countable coinitial subset $\left\{\theta_{j}\right\}_{j=1}^{\infty}$ such that $\theta_{j+1}$ is not merely less $(\bmod \mathscr{W})$ than any power of $\theta_{j}$, but in fact is less $(\bmod \mathscr{W})$ than the composition with $\theta_{j}$ of any non-decreasing $f: \mathbf{R} \rightarrow \mathbf{R}\left(f \circ \theta_{j}>_{\mathscr{W}} \theta_{j+1}\right)$. This is Lemma 3.2.1. 
We observe that for any $\psi, \Omega: \mathbf{N} \rightarrow(0,1]$,

$$
\begin{gathered}
\bigcup_{k=1}^{\infty}\left[\psi^{-1}((0,1 / k])-\Omega^{-1}((0,1 / k])\right] \subset\{n \mid \psi(n)<\Omega(n)\} \\
\subset \bigcup_{k=1}^{\infty}\left[\psi^{-1}((0,1 / k])-\Omega^{-1}((0,1 /(k+1)])\right] .
\end{gathered}
$$

Therefore $f \circ \theta_{j}>_{\mathscr{W}} \theta_{j+1}$ for all non-decreasing $f: \mathbf{R}^{+} \rightarrow \mathbf{R}^{+}$is equivalent to

$$
\bigcup_{k=1}^{\infty}\left\{A_{j+1, \sigma(k)}-A_{j, k}\right\} \in \mathscr{W} \quad \text { for all } \sigma: \mathbf{N} \rightarrow \mathbf{N} .
$$

There are two main steps in constructing the ultrafilter $\mathscr{W}$.

The first step is by far the hardest. In the first step we construct a filter $\mathscr{H}$ and a countable subset of $l^{\infty},\left\{\theta_{j}\right\}_{j=1}^{\infty}$ such that:

(1) $\mathscr{H}$ contains the family of sets $\left\{A_{j, k}\right\}_{j=1}^{\infty} \infty$, with $A_{j, k}=$ $\theta_{j}^{-1}((0,1 / k])$.

(2) $\mathscr{H}$ contains the family of sets $\left\{\bigcup_{k=1}^{\infty}\left[A_{j+1, \sigma(k)}-A_{j, k}\right]\right\}$, for all $\sigma: \mathbf{N} \rightarrow \mathbf{N}$ and for all $j \in \mathbf{N}$.

(3) For any set $B_{j}$ with $\left(B_{j}-A_{j, k}\right)$ finite for all $k \in \mathbf{N}, B_{j}^{C} \in \mathscr{H}$.

(4) For any $\psi \in l^{\infty}$ either $|\psi|>_{\mathscr{H}} \theta_{j}$ for some $j \in \mathbf{N}$ or

for some filter $\mathscr{G}$ extending $\mathscr{H}, \psi$ converges $(\bmod \mathscr{G})$.

The last property of $\mathscr{H}$ could be rephrased (and this is the form of the property that we work with):

For any nested sequence of sets $\left\{C_{m}\right\}_{m=1}^{\infty}$ either

$(\alpha)$ There exists $G \in \mathscr{H}$ and $j \in \mathbf{N}$ such that $G \cap C_{m} \subset A_{j, m}$ for all $m \in \mathbf{N}$, or

( $\beta$ ) There exists $C \subset \mathbf{N}$ such that $C-C_{m}$ is finite for all $m \in \mathbf{N}$, and for all $H \in \mathscr{H}, C \cap H \neq \varnothing$.

If $\mathscr{H}$ has all four of these properties we say that $\mathscr{H}$ is combed by the family of sets $\mathscr{A}=\left\{A_{j, k}\right\}_{j=1}^{\infty} \underset{k=1}{\infty}$.

In the second step we extend $\mathscr{H}$ to an ultrafilter $\mathscr{W}$, taking care that $\mathscr{W}$ satisfies property (4). (Properties (1), (2), and (3) are true for all extensions of $\mathscr{H}$.) For the ultrafilter $\mathscr{W}$, property (4) will mean that, given $\psi \in l^{\infty}$, either $|\psi|>_{\mathscr{W}} \theta_{j}$ for some $j$ or $\psi$ converges $(\bmod \mathscr{W}) . \quad\left\{\left[\theta_{j}\right]\right\}_{j=1}^{\infty}$, then, will be the desired countable coinitial subset.

LEMMA 3.2.1. Let $\mathscr{W}$ be a non-principal ultrafilter on $\mathbf{N}$. Let $\left\{\theta_{j}\right\}_{j=1}^{\infty}$ be a countable coinitial subset of $\left(l_{0, \mathscr{W}}-c_{0, \mathscr{W}}\right)_{+}$. Then for 
each $j$, there exists $m(j) \in \mathbf{N}$ such that, for all non-decreasing functions $f: \mathbf{R}^{+} \rightarrow \mathbf{R}^{+}, f \circ \theta_{j}>_{\mathscr{W}} \theta_{m(j)}$.

Proof. Assume false. Let there exist $\theta_{j}$ such that for each $\theta_{m}$, there exists $f_{m}$ such that

$$
\theta_{m}>\mathscr{W} f_{m} \circ \theta_{j}
$$

Define $f$, for $1 /(n+1)<x<1 / n$,

$$
f(x)=\bigwedge_{m=1}^{n} f_{m}(x) .
$$

Then, for all $m$,

$$
\theta_{m} \underset{\mathscr{W}}{>} f \circ \theta_{j}
$$

Claim. $f \circ \theta_{j} \notin c_{0}, \mathscr{W}$.

Proof of Claim. Let $A \in \mathscr{W}$ be such that $\left.f \circ \theta_{j}\right|_{A}$ converges to zero. For $b, c>0$, if $f(b)=c$, then $f \circ \theta_{j}(n)<c / 2$ implies $\theta_{j}(n)<b$. Hence $\left.\theta_{j}\right|_{A}$ converges to zero, contrary to hypothesis.

This claim and the fact that

$$
\theta_{m} \underset{\mathscr{W}}{\longrightarrow} f \circ \theta_{j} \text { for all } m
$$

together contradict the coinitiality of $\left\{\theta_{m}\right\}_{m=1}^{\infty}$.

Proposition 3.2.2. Let $\mathscr{W}$ be a non-principal ultrafilter on $\mathbf{N}$. Then the following are equivalent:

(i) There exists a (non-empty), countable, coinitial subset of $\left(l_{0, \mathscr{W}} / c_{0, \mathscr{W}}\right)_{+}$.

(ii) There exists a countable family of subsets $\left\{A_{j, k}\right\}_{j=1}^{\infty} \sum_{k=1}^{\infty} \subset \mathscr{W}$ such that

(1) $A_{j, k} \subset A_{j+1, k}$,

(2) $A_{j, k+1} \subset A_{j, k}$,

(3) for all sequences $\sigma: \mathbf{N} \rightarrow \mathbf{N}$, and for all $j$,

$$
\left[\bigcup_{k=1}^{\infty}\left(A_{j+1, \sigma(k)}-A_{j, k}\right)\right] \in \mathscr{W},
$$

(4) for $B$ a subset of $\mathbf{N}$, if for some $j \in \mathbf{N}, B \cup A_{j, k}$ has finite complement for all $k \in \mathbf{N}$, then $B \in \mathscr{W}$, and

(5) given a countable family of sets $\left\{C_{k}\right\}_{k=1}^{\infty}$, such that 
(a) $C_{k+1} \subset C_{k}$,

(b) $C_{k} \in \mathscr{W}$ for all $k$, and

(c) for all $j,\left[\bigcup_{k=1}^{\infty}\left(C_{k}-A_{j, k}\right)\right] \in \mathscr{W}$,

there exists $C \in \mathscr{W}$ such that $\left(C-C_{k}\right)$ is finite for all $k$.

Proof. (i) $\rightarrow$ (ii). Let $\left\{\left[\theta_{j}\right]\right\}_{j=1}^{\infty}$ be a countable coinitial subset of $\left(l_{0, \mathscr{W}} / c_{0}, \mathscr{W}\right)_{+}$. Let $\theta_{j}(n) \geq \theta_{j+1}(n)>0$. By Lemma 3.2.1, for each $j$ there exists $m(j) \in \mathbf{N}$, with

$$
\theta_{j} \underset{\mathscr{W}}{>} f \circ \theta_{m(j)}
$$

for all non-decreasing $f: \mathbf{R}^{+} \rightarrow \mathbf{R}^{+}$. Without loss of generality let $m(j)=j+1$. Then let

$$
A_{j, k}=\left\{n \mid 1 / k \geq \theta_{j}(n)\right\} .
$$

It can be checked that $\left\{A_{j, k}\right\}_{j=1}^{\infty} \infty$ has the desired properties.

(ii) $\rightarrow\left(\right.$ i). We define $\theta_{j}$ :

$$
\theta_{j}(n)= \begin{cases}1 & \text { if } n \notin A_{j, 2}, \\ 1 / k & \text { if } n \in A_{j, k}-A_{j, k+1}, \\ 0 & \text { if } n \in \bigcap_{k=1}^{\infty} A_{j, k} .\end{cases}
$$

Clearly $\left\{\left[\theta_{j}\right]\right\}$ is a countable coinitial subset of $\left(l_{0, \mathscr{W}} / c_{0}, \mathscr{W}\right)_{+}$. This completes the proof.

The rest of this section will be devoted to constructing a $\mathscr{W}$ containing a family of sets such as in Proposition 3.2.2.

Definition 3.2.3. Given a family $\mathscr{A}=\left\{A_{j, k}\right\}_{j=1}^{\infty} \underset{k=1}{\infty}$ of subsets of $\mathbf{N}$, such that

(a) $A_{j, k} \subset A_{j+1, k}$,

(b) $A_{j, k+1} \subset A_{j, k}$,

a filter $\mathscr{H}$ is combed by $\mathscr{A}$ if

$(\alpha)$ For all maps $\sigma: \mathbf{N} \rightarrow \mathbf{N}, \bigcup_{k=1}^{\infty}\left[A_{j+1, \sigma(k)}-A_{j, k}\right] \in \mathscr{H}$.

$(\beta)$ Given a nested sequence of subsets of $\mathbf{N},\left\{C_{m}\right\}$, either (one) There exists $C \subset \mathbf{N}$ such that $C-C_{m}$ is finite for all $m \in \mathbf{N}$ and for every $H \in \mathscr{H}, C \cap H \neq \varnothing$.

(two) There exists $G \in \mathscr{H}$ and $j \in \mathbf{N}$ such that $\left(G \cap C_{m}\right) \subset$ $A_{j, m}$ for all $m \in \mathbf{N}$.

Lemma 3.2.4. Let $\mathscr{A}$ be as in Definition 3.2.3. Let a filter $\mathscr{H}$ be combed by $\mathscr{A}$. Then the following are satisfied: 
(a) For all $j \in \mathbf{N},\left[\bigcap_{k=1}^{\infty} A_{j, k}\right]^{c} \in \mathscr{H}$.

(b) If $B \subset \mathbf{N}$ is such that for some $j \in \mathbf{N}, B \cup A_{j, k}$ has finite complement for all $k \in \mathbf{N}$, then $B \in \mathscr{H}$.

Proof. (a) Let $\sigma(k)=k$. Then given $x \in \bigcap_{k=1}^{\infty} A_{j, k}, x \notin\left[A_{j+1, k}-\right.$ $\left.A_{j, k}\right]$ for any $k$. Hence

$$
\left[\bigcup_{k=1}^{\infty}\left(A_{j+1, k}-A_{j, k}\right)\right] \subset\left[\bigcap_{k=1}^{\infty} A_{j, k}\right]^{c} .
$$

(b) For $B$ and $j$ as in the hypothesis, $B^{c}-A_{j, k}$ is finite for all $k$. Define

$$
\sigma(k)=1+\max \left\{m \mid\left(B^{c}-A_{j, k}\right) \cap\left[\bigcap_{r=1}^{\infty} A_{j+1, r}\right]^{c} \cap A_{j+1, m} \neq \varnothing\right\}
$$

Clearly,

$$
\left[\bigcap_{r=1}^{\infty} A_{j+1, r}\right]^{c} \cap B^{c} \cap\left[\bigcup_{k=1}^{\infty}\left(A_{j+1, \sigma(k)}-A_{j, k}\right)\right]=\varnothing
$$

and

Hence $B \in \mathscr{H}$.

$$
\left[\bigcup_{k=1}^{\infty}\left(A_{j+1, \sigma(k)}-A_{j, k}\right)\right] \cap\left[\bigcap_{r=1}^{\infty} A_{j+1, r}\right]^{c} \subset B .
$$

The above lemma shows that property (4) of Proposition 3.2.2 is really superfluous.

REMARK 3.2.5. For a non-principal ultrafilter $\mathscr{W}$ on $\mathbf{N}$, the following are equivalent:

(i) $\left(l_{0, \mathscr{W}} / \mathcal{c}_{0}, \mathscr{W}\right)_{+}$has a countable coinitial subset.

(ii) There exists a family of sets $\mathscr{A}=\left\{A_{j, k}\right\}_{j=1}^{\infty} \infty_{k=1}^{\infty}$ as in Definition 3.2.3 such that $\mathscr{W}$ is combed by $\mathscr{A}$.

Our next step is to find a family $\mathscr{A}=\left\{A_{j, k}\right\}_{j=1}^{\infty} \operatorname{lol}_{k=1}^{\infty}$ and a filter $\mathscr{H}$ that is combed by $\mathscr{A}$. Later, we shall extend $\mathscr{H}$ to an ultrafilter $\mathscr{W}$ which is also combed by $\mathscr{A}$.

Notation 3.2.6. We let $r_{j}$ denote the $j$ th prime.

Definition 3.2.7. We define a family of functions $\left\{v_{j}: \mathbf{N} \rightarrow \mathbf{N}\right\}$, by

$$
v_{j}(n)=k \quad \text { if } r_{j}^{k} \mid n \text { and } r_{j}^{k+1} \nmid n \text {. }
$$


We are now ready to construct $\mathscr{A}=\left\{A_{j, k}\right\}_{j=1}^{\infty} \infty$.

Notation 3.2.8. $u_{j, k}, w_{j, k}$, and $A_{j, k}$ will denote the following sets

$$
\begin{aligned}
u_{j, k} & =\left\{n \mid v_{j}(n)=k\right\}, \\
w_{j, k} & =\bigcup_{m \geq k} u_{j, m}=\left\{n \mid v_{j}(n) \geq k\right\}, \\
A_{j, k} & =\bigcup_{i=1}^{j} w_{i, k} .
\end{aligned}
$$

Clearly $A_{j, k} \subset A_{j+1, k}$ and $A_{j, k+1} \subset A_{j, k}$.

Now we are ready to construct the filter $\mathscr{H}$.

Notation 3.2.9. For $\mathscr{A}$ as above, given $j \in \mathbf{N}$ and a map $\sigma: \mathbf{N} \rightarrow$ $\mathbf{N}, F_{j, \sigma}$ will denote the set

$$
F_{j, \sigma}=\left[\bigcup_{k=1}^{\infty}\left(A_{j+1, \sigma(k)}-A_{j, k}\right)\right] .
$$

Lemma 3.2.10. Let $A_{j, k}, j \in \mathbf{N}, k \in \mathbf{N}$ be as in Notation 3.2.8 and let $F_{j, \sigma}, j \in \mathbf{N}, \sigma \in \mathbf{N}^{\mathbf{N}}$ be as in Notation 3.2.9. Then the family

$$
\left\{A_{j, k}\right\}_{\substack{j \in \mathbf{N} \\ k \in \mathbf{N}}} \cup\left\{F_{j, \sigma}\right\}_{\substack{j \in \mathbf{N} \\ \sigma \in \mathbf{N}^{\mathbf{N}}}}
$$

has the finite intersection property.

Proof. For a finite collection of sets $\left\{A_{j_{1}, k_{t}}\right\}_{i=1}^{m}$, if we let $k^{\prime}=$ $\max \left\{k_{i}\right\}$, then $A_{1, k^{\prime}} \subset \bigcap_{i=1}^{m} A_{j_{l}, k_{i}}$. For all fixed $j$ and a finite collection of functions $\left\{\sigma_{i}: \mathbf{N} \rightarrow \mathbf{N}\right\}_{i=1}^{m}$, if we let $\sigma=\sigma_{1} \vee \sigma_{2} \vee \cdots \vee \sigma_{m}$, then $F_{j, \sigma} \subset \bigcap_{i=1}^{m} F_{j, \sigma_{i}}$. Therefore, we need only consider sets of the form

$$
H=A_{1, k} \cap \bigcap_{i=1}^{m} F_{j_{\imath}, \sigma_{i}}
$$

where $j_{i+1}>j_{i}$ for all $i<m$. We let $k_{0}=k$ and $k_{i}=1+\sigma_{i}\left(k_{i-1}+1\right)$. Then

$$
\left[2^{k} \cdot \prod_{i=1}^{m} r_{j_{\imath}}^{k_{t}}\right] \in H
$$

This completes the proof.

Notation 3.2.11. From now on $\mathscr{H}$ will denote the filter generated by

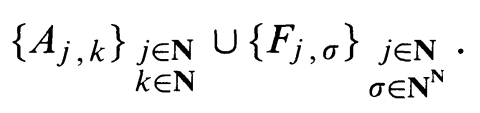


We need to show that $\mathscr{H}$ is combed by

$$
\mathscr{A}=\left\{A_{j, k}\right\} \underset{j \in \mathbf{N}}{\substack{j \in \mathbf{N} \\ \text {. }}}
$$

REMARK 3.2.12. Observe that for all $j \in \mathbf{N}$ and $k \in \mathbf{N}$,

$$
A_{j, k} \cap\left[\bigcup_{m=1}^{\infty}\left(A_{j, m}-A_{j-1, m}\right)\right] \subset w_{j, k},
$$

so $w_{j, k} \in \mathscr{H}$.

In order to prove that $\mathscr{H}$ is combed by $\mathscr{A}$ : We will define a family of properties for subsets of $\mathbf{N},\{M(i, j)\}$, such that if $K \subset \mathbf{N}$ has property $M(i, j)$ for all $i \in \mathbf{N}$ and $j \in \mathbf{N}$ then the family of sets $\mathscr{H} \cup\{K\}$ has the finite intersection property. We will also define a family of properties $\{P(i, j)\}$ for nested sequence of subsets of $\mathbf{N}$, such that if $\left\{C_{m}\right\}_{m=1}^{\infty}$ does not have property $P(i, j)$ for all $i, j \in \mathbf{N}$ then there exists $G \in \mathscr{H}$ and $r \in \mathbf{N}$ such that $C_{m} \cap G \subset A_{r, m}$ for all $m$. On the other hand, if $\left\{C_{m}\right\}_{m=1}^{\infty}$ does have property $P(i, j)$ for all $i, j \in \mathbf{N}$ then there exists $C$ such that $C-C_{m}$ is finite for all $m \in \mathbf{N}$ and $C$ has property $M(i, j)$ for all $i, j \in \mathbf{N}$. Hence $\mathscr{H} \cup\{C\}$ has the finite intersection property.

Definition 3.2.13. For

$$
\left\{A_{j, k}\right\}_{\substack{j \in \mathbf{N} \\ k \in \mathbf{N}}}
$$

as in Notation 3.2.8, a set $K \subset \mathbf{N}$ has property $M(1, j)$ if

$$
\left\{k \mid K \cap u_{j, k} \neq \varnothing\right\}
$$

is an infinite set.

We define "the property $M(i, j)$ " inductively.

$$
\begin{aligned}
& \text { Definition 3.2.14. For } i \geq 2 \text { a set } K \subset \mathbf{N} \text { has property } M(i, j) \\
& \text { if }
\end{aligned}
$$

$$
\left\{k \mid K \cap u_{j, k} \text { has property } M(i-1, j+1)\right\}
$$

is an infinite set.

We shall next show (Proposition 3.2.17) that if $K$ has property $M(i, j)$ for all $i \in \mathbf{N}$ and $j \in \mathbf{N}$, then $K$ has non-empty intersection with each set $H \in \mathscr{H}$. (In fact, we shall show that for $H \in \mathscr{H}, H \cap K$ also has property $M(i, j)$ for all $i \in \mathbf{N}, j \in \mathbf{N}$.) Then $\mathscr{H} \cup\{K\}$ has the finite intersection property. 
LEMMA 3.2.15. Let $K$ have property $M(i, j)$ for all $i \in \mathbf{N}$ and all $j \in \mathbf{N}$. Then for all $M \in \mathbf{N}$ and $n \in \mathbf{N}, K \cap A_{m, n}$ also has property $M(i, j)$ for all $i \in \mathbf{N}$ and $j \in \mathbf{N}$.

Proof. It is sufficient to show that $K \cap A_{m, n}$ has property $M(i, 1)$ for all $i \geq 2$. Since $K \cap A_{1, n} \subset K \cap A_{m, n}$, it is sufficient to show that $K \cap A_{1, n}$ has property $M(i, 1)$ for all $i \geq 2$. So let $K$ have property $M(i, 1)$ for some $i \geq 2$. Then

$$
\left\{k \mid K \cap u_{1, k} \text { has property } M(i-1,2)\right\}
$$

is an infinite set. But then

$$
\left\{k \geq n \mid K \cap u_{1, k} \text { has property } M(i-1,2)\right\}
$$

is also an infinite set, and $K \cap A_{1, n}$ also has property $M(i, 1)$.

LEмMA 3.2.16. Let $F_{m, \sigma}$ be as in Notation 3.2.9. If $K \subset \mathbf{N}$ has property $M(i, j)$ for all $i \in \mathbf{N}$ and $j \in \mathbf{N}$, then for all $m \in \mathbf{N}$ and $\sigma \in \mathbf{N}^{\mathbf{N}}, K \cap F_{m, \sigma}$ has property $M(i, j)$ for all $i \in \mathbf{N}$ and $j \in \mathbf{N}$.

Proof. It clearly suffices to show that $K \cap F_{m, \sigma}$ has property $M(i, j)$ for all $i \geq 3$ and for all $j \leq m$. Let $K \cap u_{m, k}$ have property $M(i-1, m+1)$. Then

$$
\left\{p \mid K \cap u_{m, k} \cap u_{m+1, p} \text { has property } M(i-2, m+2)\right\}
$$

is an infinite set. But then

$$
\left\{p>\sigma(k+1) \mid K \cap u_{m, k} \cap u_{m+1, p} \text { has property } M(i-2, m+2)\right\}
$$

is also an infinite set, and $K \cap u_{m, k} \cap F_{m, \sigma}$ has the property $M(i-1, m+1)$. Thus

$$
\begin{aligned}
& \left\{k \mid K \cap u_{m, k} \text { has property } M(i-1, m+1)\right\} \\
& \quad=\left\{k \mid K \cap u_{m, k} \cap F_{m, \sigma} \text { has property } M(i-1, m+1)\right\} .
\end{aligned}
$$

So if $K$ has property $M(i, m)$, then so does $K \cap F_{m, \sigma}$.

Claim. Given $m \geq 2$, if the pair $(d, n)$, with $m \geq d \geq 2$ and $n \geq 3$ has the property:

"If $L \subset \mathbf{N}$ has property $M(i, d)$ for some $i \geq n$, then so does $L \cap F_{m, \sigma}$, for all $\sigma: \mathbf{N} \rightarrow \mathbf{N}$,"

then the pair $(d-1, n+1)$ has the same property. 
Proof of Claim. For some $i \geq n+1$, let $J \subset \mathbf{N}$ have property $M(i, d-1)$. Given $k \in \mathbf{N}$, if $\left[J \cap u_{d-1, k}\right]$ has property $M(i-1, d)$, then by hypothesis so does $\left[J \cap u_{d-1, k} \cap F_{m, \sigma}\right]$. That is

$$
\begin{aligned}
& \left\{k \mid J \cap u_{d-1, k} \text { has property } M(i-1, d)\right\} \\
& \quad=\left\{k \mid J \cap u_{d-1, k} \cap F_{m, \sigma} \text { has property } M(i-1, d)\right\} .
\end{aligned}
$$

This proves the claim.

We have already shown that the pair $(m, 3)$ has the property in the claim; hence so does $(1, m+2)$. This proves lemma.

Proposition 3.2.17. If $K \subset \mathbf{N}$ has property $M(i, j)$ for all $i \in \mathbf{N}$ and $j \in \mathbf{N}$ then for all $H \in \mathscr{H}, K \cap H$ has property $M(i, j)$ for all $i \in \mathbf{N}$ and $j \in \mathbf{N}$. A fortiori $K \cap H \neq \varnothing$.

Proof. Follows from Lemma 3.2.15 and Lemma 3.2.16.

Definition 3.2.18. $\left\{C_{m}\right\}_{m=1}^{\infty}$, a nested sequence of subsets of $\mathbf{N}$, has property $P(1, j)$ if

$$
\left[\bigcap_{m=1}^{\infty}\left\{k \mid C_{m} \cap u_{j, k} \neq \varnothing\right\}\right]
$$

is an infinite set.

We define "the property $P(i, j)$ " inductively.

DEFINITION 3.2.19. $\left\{C_{m}\right\}_{m=1}^{\infty}$, a nested sequence of subsets of $\mathbf{N}$, has property $P(i, j)$ if

$$
\left\{k \mid\left\{C_{m} \cap u_{j, k}\right\}_{m=1}^{\infty} \text { has property } P(i-1, j+1)\right\}
$$

is an infinite set.

Proposition 3.2.20. If for some $i \in \mathbf{N}, j \in \mathbf{N}$, a nested sequence of sets $\left\{C_{m}\right\}_{m=1}^{\infty}$ does not have property $P(i, j)$ then there exist $k \in \mathbf{N}$ and $\sigma \in \mathbf{N}^{\mathbf{N}}$ such that for all $m \in \mathbf{N}$

$$
\left[A_{j, k} \cap C_{m} \cap \bigcap_{q=j}^{j+i-1} F_{q, \sigma}\right] \subset A_{j+i, m} .
$$

Proof. We proceed by induction.

$i=1$. Given $j \in \mathbf{N}$, such that $\left\{C_{m}\right\}_{m=1}^{\infty}$ does not have property $P(1, j)$ we let

$$
k^{\prime}=1+\max \left\{k \mid C_{m} \cap u_{j, k} \neq \varnothing \text { for all } m \in \mathbf{N}\right\} .
$$


Then, for $k \geq k^{\prime}$, we define

$$
m(k)=\max \left\{m \mid C_{m} \cap u_{j, k} \neq \varnothing\right\} .
$$

We define $\sigma: \mathbf{N} \rightarrow \mathbf{N}$ by

$$
\sigma(k)=1+\max _{k^{\prime} \leq r \leq k}\{m(r)\} .
$$

We shall show that

$$
A_{j, k^{\prime}} \cap C_{m} \cap\left[\bigcup_{k=1}^{\infty}\left(A_{j+1, \sigma(k)}-A_{j, k}\right)\right] \subset A_{j+1, m} .
$$

Letting

$$
t(m)=\min \left\{k \geq k^{\prime} \mid C_{m} \cap u_{j, k} \neq \varnothing\right\}
$$

it follows that

$$
\begin{aligned}
A_{j, k^{\prime}} & \cap C_{m} \cap\left[\bigcup_{k=1}^{\infty}\left(A_{j+1, \sigma(k)}-A_{j, k}\right)\right] \\
& \subset C_{m} \cap\left[\bigcup_{k \geq t(m)+1}^{\infty}\left(A_{j+1, \sigma(k)}-A_{j, k}\right)\right] \subset A_{j+1, \sigma(t(m)+1)}
\end{aligned}
$$

Next we show that

$$
\begin{gathered}
\sigma(t(m)+1) \geq m . \\
\sigma(t(m)+1)=1+\max _{k^{\prime} \leq r \leq t(m)+1}\{m(r)\} \\
=1+\max \left\{s \mid \exists r, k^{\prime} \leq r \leq t(m)+1 ; C_{s} \cap u_{j, r} \neq \varnothing\right\} \\
\geq 1+\max \left\{s \mid C_{s} \cap u_{j, t(m)} \neq \varnothing\right\} \geq 1+m>m .
\end{gathered}
$$

$i \geq 2$. Assume that the proposition is true for all $j \in \mathbf{N}$ and all $d<i$. Let $\left\{C_{m}\right\}_{m=1}^{\infty}$ not have property $P(i, j)$. That is,

$$
\left\{k \mid\left\{C_{m} \cap u_{j, k}\right\}_{m=1}^{\infty} \text { has property } P(i-1, j+1)\right\}
$$

is a finite set.

Let

$$
k^{\prime}=1+\max \left\{k \mid\left\{C_{m} \cap u_{j, k}\right\}_{m=1}^{\infty} \text { has property } P(i-1, j+1)\right\} .
$$

So, for $k \geq k^{\prime}$, by induction there exist $z(k) \in \mathbf{N}$ and a map $\sigma_{k}: \mathbf{N} \rightarrow$ $\mathbf{N}$ such that for all $m \in \mathbf{N}$

$$
C_{m} \cap u_{j, k} \cap A_{j+1, z(k)} \cap\left[\bigcap_{s=j+1}^{j+i-1} F_{s, \sigma_{k}}\right] \subset A_{j+i, m} .
$$


We define the map $\sigma: \mathbf{N} \rightarrow \mathbf{N}$ by

$$
\sigma(k)=\max \left\{k, \max _{k^{\prime} \leq r \leq k}\{z(r)\}, \max _{k^{\prime} \leq r \leq k}\left\{\sigma_{r}(k)\right\}\right\} .
$$

We shall show that

$$
A_{j, k^{\prime}} \cap C_{m} \cap\left[\bigcap_{\lambda=j}^{j+i-1} F_{\lambda, \sigma}\right] \subset A_{j+i, m}
$$

This will complete the proof of the proposition.

Let $x \in C_{m} \cap u_{j, k}$ for some $k \geq k^{\prime}$. Now observe that for $d \geq k$,

$$
\sigma(d) \geq \sigma_{k}(d) \text { and } \sigma(d) \geq k .
$$

Hence

$$
\sigma \circ \sigma(d) \geq \sigma_{k} \circ \sigma_{k}(d)
$$

and

$$
[\sigma]^{q}(d) \geq\left[\sigma_{k}\right]^{q}(d)
$$

Therefore

$$
u_{j, k} \cap\left[\bigcap_{\lambda=j}^{j+i-1} F_{\lambda, \sigma}\right] \subset A_{j+1, z(k)} \cap\left[\bigcap_{s=j+1}^{j+i-1} F_{s, \sigma_{k}}\right] .
$$

Thus, for all $k \geq k^{\prime}$

$u_{j, k} \cap C_{m} \cap\left[\bigcap_{\lambda=j}^{j+i-1} F_{\lambda, \sigma}\right] \subset A_{j+1, z(k)} \cap C_{m} \cap u_{j, k} \cap\left[\bigcap_{s=j+1}^{j+i-1} F_{s, \sigma_{k}}\right] \subset A_{j+i, m}$

This completes the proof of the proposition.

LEMMA 3.2.21. Let a nested sequence of sets, $\left\{C_{m}\right\}_{m=1}^{\infty}$, have property $P(i, j)$ for all $i \in \mathbf{N}$ and $j \in \mathbf{N}$. Then there exists $C \subset \mathbf{N}$ such that

(1) $C-C_{m}$ is finite for all $m \in \mathbf{N}$.

(2) $C$ has property $M(i, j)$ for all $i \in \mathbf{N}$ and $j \in \mathbf{N}$.

Proof. Clearly, if $C$ has property $M(i, 1)$ for all $i \in \mathbf{N}$ then it has property $M(i, j)$ for all $i \in \mathbf{N}$ and $j \in \mathbf{N}$.

We choose an increasing function $\theta: \mathbf{N} \rightarrow \mathbf{N}$ such that

$$
\left\{C_{m} \cap u_{1, \theta(i)}\right\}_{m=1}^{\infty}
$$

has property $P(i, 2)$. 
Next, we choose a family of maps

$$
\left\{\psi_{i}: \mathbf{N}^{i} \rightarrow \mathbf{N}^{i}\right\}_{i=1}^{\infty}
$$

such that, if

$$
\left(b_{1}, \ldots, b_{i}\right) \in \text { range } \psi_{i}
$$

then for $r<i$

$$
\left\{u_{i, \theta(i)} \cap C_{m} \cap\left[\bigcap_{d=1}^{r} u_{d+1, b_{d}}\right]\right\}_{m=1}^{\infty}
$$

has property $P(i-r, 1+r)$, and

$$
u_{1, \theta(i)} \cap C_{m} \cap\left[\bigcap_{d=1}^{i} u_{d+1, b_{d}}\right] \neq \varnothing
$$

for all $m \in \mathbf{N}$.

We construct $C$ in the following manner: Given $m \in \mathbf{N}$, for each $r \leq m$, and for each $r$-tuple whose entries $\left(a_{1}, \ldots, a_{r}\right)$ are less than or equal to $m$, letting

$$
\psi_{r}\left(a_{1}, \ldots, a_{r}\right)=\left(b_{1}, \ldots, b_{r}\right)
$$

we choose an $x$,

$$
x \in C_{m} \cap u_{1, \theta(r)} \cap\left[\bigcap_{d=1}^{r} u_{d+1, b_{d}}\right] .
$$

We let $C$ be the set of all these $x$ 's. $C$ clearly has property $M(i, 1)$ for all $i \in \mathbf{N}$, and clearly $C-C_{m}$ is finite for all $m \in \mathbf{N}$.

REMARK 3.2.22. Lemma 3.2.21 shows that for a nested sequence of sets $\left\{C_{m}\right\}_{m=1}^{\infty}$, there will exist $G \in \mathscr{H}$ and $j \in \mathbf{N}$ such that $C_{m} \cap G \subset$ $A_{j, m}$ for all $m \in \mathbf{N}$, if and only if for some $i \in \mathbf{N}$ and $j \in \mathbf{N}$, $\left\{C_{m}\right\}_{m=1}^{\infty}$ does not have property $P(i, j)$. Further, by considering the sequence of sets $\left\{C_{m}\right\}_{m=1}^{\infty}$ with $C_{m}=C$ for all $m \in \mathbf{N}$, we see that there exists $G \in \mathscr{H}$ with $G \cap C=\varnothing$ if and only if, for some $i \in \mathbf{N}$ and $j \in \mathbf{N}, C$ does not have property $M(i, j)$

Proposition 3.2.23. Let $\mathscr{A}$ be as in Notation 3.2.8. Let $\mathscr{H}$ be as in Notation 3.2.11. Then $\mathscr{H}$ is combed by $\mathscr{A}$.

Proof. Trivially, in fact by definition of $\mathscr{H}$,

$$
\bigcup_{k=1}^{\infty}\left[A_{j+1, \sigma(k)}-A_{j, k}\right] \in \mathscr{H} \quad \text { for all } \sigma: \mathbf{N} \rightarrow \mathbf{N},
$$


and $\mathscr{H}$ satisfies $(\alpha)$ of Definition 3.2.3. either

Given a nested sequence of sets $\left\{C_{m}\right\}_{m=1}^{\infty}$, by Proposition 3.2.20,

(1) There exists $H \in \mathscr{H}$ and $j \in \mathbf{N}$ such that $C_{m} \cap H \subset A_{j, m}$ for all $m \in \mathbf{N}$ or

(2) $\left\{C_{m}\right\}_{m=1}^{\infty}$ has property $P(i, j)$ for all $i \in \mathbf{N}$ and $j \in \mathbf{N}$.

(1) is condition ( $\beta$ one) of Definition 3.2.3, so we assume that (2) holds. Then by Lemma 3.2.21, there exists $C \subset \mathbf{N}$ such that, for all $m \in \mathbf{N}, C-C_{m}$ is a finite set, and such that $C$ has property $M(i, j)$ for all $i \in \mathbf{N}$ and $j \in \mathbf{N}$. By Proposition 3.2.17, for such a $C$, given any $H \in \mathscr{H}, C \cap H \neq \varnothing$. This is condition ( $\beta$ two) of Definition 3.2.3, and so the proof is complete.

We next show that if a filter $\mathscr{H}$ is combed by a family of sets $\mathscr{A}$, then so is every countable extension of $\mathscr{H}$.

LeMmA 3.2.24. Let $\mathscr{A}$ be as in Notation 3.2.8. Let $\mathscr{H}$ be as in Notation 3.2.11. Let $\left\{D_{\alpha}\right\}$ be a countable family of subsets of $\mathbf{N}$ such that $\mathscr{H} \cup\left\{D_{\alpha}\right\}$ has the finite intersection property. Let $\mathscr{G}$ be the filter generated by $\mathscr{H} \cup\left\{D_{\alpha}\right\}$. Then $\mathscr{G}$ is combed by $\mathscr{A}$.

Proof. Order $\left\{D_{\alpha}\right\}$ as a sequence $\left\{D_{n}\right\}$. Let $E_{i}=\bigcap_{n=1}^{i} D_{n}$. Clearly $E_{i} \in \mathscr{G}$ for all $i \in \mathbf{N}$. Also clearly, given a set $K \subset \mathbf{N}$ such that for all $H \in \mathscr{H}$ and all $i \in \mathbf{N}, K \cap H \cap E_{i} \neq \varnothing$, we have that for all $G \in \mathscr{G}$, $K \cap G \neq \varnothing$.

Let $\left\{C_{m}\right\}_{m=1}^{\infty}$ be a nested sequence of subsets of $\mathbf{N}$ such that

(a) for all $G \in \mathscr{G}$ and all $m \in \mathbf{N}, G \cap C_{m} \neq \varnothing$ and

(b) there does not exist $G \in \mathscr{G}$ such that for some $j \in \mathbf{N}$

$$
G \cap C_{m} \subset A_{j, m} \text { for all } m \in \mathbf{N} \text {. }
$$

Clearly $\left\{C_{m}\right\}_{m=1}^{\infty}$ also has these properties with respect to $\mathscr{H}$ and, also clearly, for all $i \in \mathbf{N}$; so does the sequence of sets

$$
\left\{C_{m}^{(i)}\right\}_{m=1}^{\infty}=\left\{\left(C_{m} \cap E_{i}\right)\right\}_{m=1}^{\infty}
$$

Thus for each $i \in \mathbf{N}$, there exists $C^{(i)} \subset E_{i}$ such that $C^{(i)}-\left[C_{m} \cap E_{i}\right]$ is finite for all $m \in \mathbf{N}$ and, for all $H \in \mathscr{H}$

$$
C^{(i)} \cap H \neq \varnothing \text {. }
$$

We let

$$
C^{\#}=\bigcup_{i=1}^{\infty}\left[C^{(i)} \cap C_{i}\right] .
$$


Clearly

$$
C^{\#}-C_{m} \subset \bigcup_{i=1}^{m-1}\left[C^{(i)}-C_{m}^{(i)}\right]
$$

and so $C^{\#}-C_{m}$ is a finite set for all $m \in \mathbf{N}$. Given $H \in \mathscr{H}$ and $E_{i}$,

$$
C^{(i)} \cap C_{i} \cap H \subset H \cap E_{i} \cap C^{\#}
$$

and

$$
C^{(i)} \cap C_{i} \cap H \neq \varnothing
$$

hence

$$
H \cap E_{i} \cap C^{\#} \neq \varnothing
$$

This completes the proof.

Clearly the above lemma would hold for any family $\mathscr{A}$ as in Definition 3.2 .3 and any filter $\mathscr{H}$ combed by $\mathscr{A}$.

Now comes the main result of this section. The ultrafilter $\mathscr{W}$, of course, is not a countable extension of $\mathscr{H}$, so we use a different method, a fairly standard trick, to construct a $\mathscr{W}$ combed by $\mathscr{A}$.

THEOREM 3.2.25. There exists an ultrafilter $\mathscr{W}$ on $\mathbf{N}$ such that $\left(l_{0, \mathscr{W}} / c_{0}, \mathscr{W}\right)_{+}$has a (non-empty) countable coinitial subset.

Proof. By Remark 3.2.5, it is sufficient to show that the $\mathscr{W}$ is combed by $\mathscr{A}$,

$$
\mathscr{A}=\left\{A_{j, k}\right\}_{j \in \mathbf{N}, k \in \mathbf{N}}
$$

where $A_{j, k}$ is as in Notation 3.2.8.

By Proposition 3.2.23, $\mathscr{H}$ is combed by $\mathscr{A}$ and by Lemma 3.2.24 so are all countable extensions of $\mathscr{H}$.

We complete the proof by induction. We shall simultaneously construct a family of filters $\left\{\mathscr{H}_{\alpha}\right\}_{\alpha<\omega_{1}}$, and a family of subsets of $\mathbf{N}$, $\left\{E_{\alpha}\right\}_{\alpha<\omega_{1}}$.

We shall construct the $\mathscr{H}_{\alpha}$ 's in the standard manner. We let $\mathscr{H}_{1}=$ $\mathscr{H}$. For each $\alpha$, when we have chosen $E_{\alpha}$ we let $\mathscr{H}_{\alpha+1}$ be the filter generated by $\mathscr{H}_{\alpha} \cup\left\{E_{\alpha}\right\}$. For a limit ordinal $\lambda$, we let

$$
\mathscr{H}_{\lambda}=\bigcup_{\alpha<\lambda} \mathscr{H}_{\alpha} .
$$

Finally,

$$
\mathscr{W}=\bigcup_{\alpha<\omega_{1}} \mathscr{H}_{\alpha} .
$$


As we observed earlier, for $\alpha<\omega_{1}, \mathscr{H}_{\alpha}$ is combed by $\mathscr{A}$. We also observed that Lemma 3.2.24 does not directly yield that $\mathscr{W}$ is combed by $\mathscr{A}$. The trick then is to properly choose the $E_{\alpha}$ 's. Toward this end, we order the power set of $\mathbf{N}$ such that each subset of $\mathbf{N}$ has countably many predecessors. $\left\{D_{\alpha}\right\}_{\alpha<\omega}$, will denote the ordered set of subsets of $\mathbf{N}$. We also order the set of nested sequences of subsets of $\mathbf{N}$ such that each sequence has countably many predecessors. We denote the ordered set of nested sequences by $\left\{\left\{C_{m}\right\}_{\gamma}\right\}_{\gamma<\omega_{1}}$ and $C_{m, \gamma}$ shall denote the $m$ th set in the nested sequence $\left\{C_{r}\right\}_{\gamma}$.

To choose $E_{\alpha}$, we consider two cases.

Case 1. There exists a nested sequence $\left\{C_{m}\right\}_{\gamma} \subset H_{\alpha}$ such that

(1a) There does not exist $C \in \mathscr{H}_{\alpha}$ such that $\left(C-C_{m, \gamma}\right)$ is a finite set for all $m \in \mathbf{N}$ and

(1b) There do not exist $H \in \mathscr{H}_{\alpha}$ and $j \in \mathbf{N}$ such that $C_{m, \gamma} \cap H \subset$ $A_{j, m}$ for all $m \in \mathbf{N}$.

We let $\delta$ be the least such $\gamma$.

We let $D \subset \mathbf{N}$ be a set such that:

(\#) $\left(D-C_{m, \delta}\right)$ is a finite set for all $m \in \mathbf{N}$ and

(\#\#) For all $H \in \mathscr{H}_{\alpha}, D \cap H \neq \varnothing$.

Such a set $D$ exists since, as we said above, $\mathscr{H}_{\alpha}$ is combed by $\mathscr{A}$. Next, either $\left(D \cap D_{\alpha}\right),\left(D \cap D_{\alpha}^{c}\right)$ or both will satisfy (\#\#) above. If $\left(D \cap D_{\alpha}\right)$ satisfies (\#\#), then let $E_{\alpha}=D \cap D_{\alpha}$; otherwise, let $E_{\alpha}=D \cap D_{\alpha}^{c}$.

Case 2. No nested sequence such as described in Case 1 exists. Then let $E_{\alpha}=D_{\alpha}$ unless $D_{\alpha}^{c} \in \mathscr{H}_{\alpha}$. If $D_{\alpha}^{c} \in \mathscr{H}_{\alpha}$, then let $E_{\alpha}=D_{\alpha}^{c}$.

So, let $\left\{C_{r}\right\}_{\gamma} \subset \mathscr{H}_{\beta}$. Clearly, for $\eta=3 \max \{\gamma, \beta\}$, either there exists $C \in \mathscr{H}_{\eta}$ with $\left(C-C_{m, \gamma}\right)$ a finite set for all $m \in \mathbf{N}$, or there exists $H \in \mathscr{H}_{\eta}$ and $j \in \mathbf{N}$ such that $C_{m, \gamma} \cap H \subset A_{j, m}$ for all $m \in \mathbf{N}$.

THEOREM 3.2.26. Up to isomorphism, there are exactly ten residue class domains of $c$, the ring of real convergent sequences.

Proof. 3.2.25, 3.1.3, 3.1.5, 2.2.13, and 2.2.14.

4. Some applications to other spaces. Cherlin and Dickmann [8] showed that for any compact space $X$ with finitely many accumulation points the residue class domains of $C(X, \mathbf{R})$ are precisely those of $c$. We show that all the residue class domains of $c$ are also residue class domains of $C(X, \mathbf{R})$ for many other spaces $X$. Among these spaces are all non-discrete metric spaces. We also show that if a space $X$ 
has a compact non-discrete subset then $C(X, \mathbf{R})$ has at least 4 of the residue class domains of $c$. Finally, we show that for certain spaces $X$, for any non-maximal prime ideal $p, C(X, \mathbf{R}) / p$ has four of the residue class domains of $c$ (e.g. $X=\mathbf{N}^{*} \times \mathbf{N}^{*}$ or even $\left.X=\Pi_{1}^{k} \mathbf{N}^{*}\right)$.

Definition 4.1. A compact space $X$ with finitely many accumulations points is said to have Cantor-Bendixson rank one. A compact space $Y$ has Cantor-Bendixson rank $n+1$ if the set of accumulation points of $Y$ has Cantor-Bendixson rank $n$ (with the subspace topology).

THEOREM 4.2. Let $X$ be a compact space having Cantor-Bendixson rank one. Let $p$ be a prime ideal of $C(X, \mathbf{R})$. Then there exists $a$ prime ideal $q$ of $c$ such that $C(X, \mathbf{R}) / p \simeq c / q$.

\section{Proof. [8], 3.3.2.}

We also wish to see for which other $X$ does $C(X, \mathbf{R})$ have the residue class domains of $\S \S 2$ and 3. First, we notice:

REMARK 4.3. Let $X$ be a completely regular space. If $\mathbf{N}^{*}$ can be imbedded in $X$, then it can be $C$-imbedded. That is, every continuous function on $\mathbf{N}^{*}$ can be extended to a continuous function on $X$, rest: $C(X, \mathbf{R}) \rightarrow C\left(\mathbf{N}^{*}, \mathbf{R}\right)$ (the restriction map) is onto, and every residue class domain of $C\left(\mathbf{N}^{*}, \mathbf{R}\right) \simeq c$ is a residue class domain of $C(X, \mathbf{R})$. In particular, this holds for any nondiscrete metric space.

There are also spaces which have some of the residue class domains of $c$, but not necessarily all. For instance, if $X$ is a compact space and has a $C^{*}$ imbedded copy of $\mathbf{N}$ (that is, all bounded sequences extend to continuous functions on $X$ ) then $X$ has exactly 4 of the residue class domains of $c, \mathbf{R}$ and the domains where $l_{0, \mathscr{U}}=c_{0, \mathscr{U}}$. Observe that, except for $\mathbf{R}$, these are real closed valuation domains, see [8].

THEOREM 4.4. Let $X$ be a non-discrete pseudo-compact space. Then among the residue class domains of $C(X, \mathbf{R})$ are rings isomorphic to the following cases of $c / p$ :

(1) $\mathbf{R}$.

(2) $l_{0, \mathscr{U}}=c_{0, \mathscr{U}}$ and $\left(c_{0} / p\right)_{+}$has no countable coinitial subset.

(3) $l_{0, \mathscr{U}}=c_{0, \mathscr{U}}$ and $\left(c_{0} / p\right)_{+}$has a coinitial subset of the form $\left\{[f]^{k}\right\}$. 
(4) $l_{0, \mathscr{U}}=c_{0, \mathscr{U}}$ and $\left(c_{0} / p\right)_{+}$has a countable coinitial subset, but not as in (3) above.

Proof. We can construct a countably infinite subset $Y$ of $X$, such that every point of $Y$ shall be an isolated point of $Y$. We choose a sequence of points $\left\{y_{n}\right\}_{n=1}^{\infty}$, and a sequence of open neighborhoods $\left\{\mathscr{O}_{n}\right\}_{n=1}^{\infty}$, such that

(a) $y_{n} \in \mathscr{O}_{n}$.

(b) $\mathscr{O}_{n} \cap \mathscr{O}_{j}=\varnothing$ if $j<n$ (by symmetry, if $j \neq n$ ).

(c) $\bigcup_{n=1}^{k} \mathscr{O}_{n}$ has infinite complement for all $k$.

Let $Y=\left\{y_{n}\right\}_{n=1}^{\infty}$. We shall first show that for every convergent sequence $g$ there exists $f \in C(X, \mathbf{R})$ such that $f\left(y_{n}\right)=g(n)$. Clearly, $g=r+h$ where $r$ is a constant sequence and $h$ converges to zero. Since $h$ converges to zero it is the uniform limit of a set of sequences $\left\{h_{j}\right\}_{j=1}^{\infty}$ having finite support,

$$
h(n)=h_{j}(n) \text { for all } n \leq j .
$$

By complete regularity of $X$ we have a family of continuous functions $\left\{f_{j}\right\}_{j=1}^{\infty}$ :

$$
f_{j}(x)= \begin{cases}r+h_{j}(n) & \text { if } x=y_{n}, \\ r & \text { if } x \notin \bigcup_{n=1}^{j} \mathscr{O}_{n} .\end{cases}
$$

Their uniform limit $f$ is the desired extension of $g$. Thus, for the restriction map

$$
\text { rest: } C(X, \mathbf{R}) \rightarrow l^{\infty}
$$

defined

$$
\operatorname{rest}(f)(n)=f\left(y_{n}\right)
$$

we have

$$
c \subset \operatorname{rest}(C(X, \mathbf{R})) \subset l^{\infty} .
$$

Let $\mathscr{V}$ be a $P$-point ultrafilter and let $p$ be a prime ideal of $c$, containing

$$
p_{\mathscr{V}}=\{f \mid\{n \mid f(n)=0\} \in \mathscr{V}\} .
$$

(Recall that $p_{b}=\left\{\theta \in l^{\infty} \mid \exists f \in p ; f \equiv_{\mathscr{V}} \theta\right\}$; Notation 2.1.2.) Then

$$
c / c \cap p_{b}=l^{\infty} / p_{b}=\operatorname{rest}(C(X, \mathbf{R})) /\left(p_{b} \cap[\operatorname{rest}(C(X, \mathbf{R}))]\right),
$$

which proves the theorem. 
COROLlary 4.5. Let $X$ be a topological space, with a compact, nondiscrete subset $W$. Then $C(X, \mathbf{R})$ has the four residue class domains listed in Theorem 4.4.

REMARK 4.6. $\beta \mathbf{N}$, the Stone-Čech compactification of $\mathbf{N}$, has precisely the 4 residue class domains listed in Theorem 4.4. This does not depend on the existence of $P$-points.

LEMMA 4.7. Let $X$ be a compact metric space of finite CantorBendixson rank. Let $\mathscr{F}$ be a non-maximal prime $z$-filter on $X$. Then there exists a compact rank one set $Y \subset X$, and an ultrafilter $\mathscr{U}$ on the isolated points of $Y$, such that if $A \in \mathscr{U}$ and $F$ contains an open neighborhood of $A$ then $F \in \mathscr{F}$.

Proof. Let $X$ have Cantor-Bendixson rank $n . X^{(n-1)}$ is a rank one set.

Case 1. There exists an open neighborhood $\mathscr{O}$ of $\left(X^{(n-1)}-X^{(n)}\right)$ such that $\overline{\mathscr{O}} \notin \mathscr{F}$. then $\mathscr{O}^{c} \in \mathscr{F}$. $\mathscr{O}^{c}$ has rank $<n$. We can repeat this with $\mathscr{O}^{c}$ acting as $X$.

Case 2. For every open neighborhood $\mathscr{O}$ of $\left(X^{(n-1)}-X^{(n)}\right), \overline{\mathscr{O}} \in$ $\mathscr{F}$. Let $A \subset\left(X^{(n-1)}-X^{(n)}\right)$ and $B \subset\left(X^{(n-1)}-X^{(n)}\right), A \cap B=\varnothing$ and $A \cup B=\left(X^{(n-1)}-X^{(n)}\right)$. Let $\mathscr{P}_{A}$ be an open neighborhood of $A$ and $\mathscr{P}_{B}$ be an open neighborhood of $B$. Then $\mathscr{P}_{A} \cup \mathscr{P}_{B}$ is an open neighborhood of $\left(X^{(n-1)}-X^{(n)}\right)$, so $\left(\overline{\mathscr{P}}_{A} \cup \overline{\mathscr{P}}_{B}\right) \in \mathscr{F}$. By primality of $\mathscr{F}$, either $\overline{\mathscr{P}}_{A} \in \mathscr{F}$ or $\overline{\mathscr{P}}_{B} \in \mathscr{F}$.

This completes the proof.

THeORem 4.8. Let $X$ be a compact metric space of finite CantorBendixson rank. Let $p$ be a non-maximal prime ideal of $C(X, \mathbf{R})$. Then $C(X, \mathbf{R}) / p$ has 4 of the residue class domains of $c$.

Proof. Let $\mathscr{F}$ be the prime $z$-filter associated with $p$. We assume that there is no rank one $F \in \mathscr{F}$, (or else this is trivial). Let $Y$ be the rank one set guaranteed by Lemma 4.7. By 1.14, the ultrafilter $\mathscr{U}$ on the isolated points of $Y$ induces a prime $z$-filter $\mathscr{G}_{Y}$ on $Y$. This induces a prime $z$-filter $\mathscr{G}$ on $X$. Clearly $\mathscr{F}$ is contained in $\mathscr{G}$. Then $p_{\mathscr{F}} \subset p_{\mathscr{G}}$ and $p_{\mathscr{G}} \not \subset p$. Therefore $p \subset p_{\mathscr{G}}$ and we have

$$
\theta: C(X, \mathbf{R}) / p \rightarrow C(X, \mathbf{R}) / p_{\mathscr{G}} \simeq C(Y, \mathbf{R}) / p_{\mathscr{G}_{r}} .
$$

The result follows immediately. 
REMARK 4.9. The author showed in [19], that for any compact space of finite rank, there is a metric space of finite rank with the same set of residue class domains. This natural generalization of Theorem 4.2 means that Theorem 4.2 could be extended to all compact spaces of finite rank. It would be extended to all compact spaces of finite rank. It would be natural to support that Lemma 4.7 could be extended much further; however, as Theorem 4.10 shows below, Lemma 4.7 cannot be extended to $[0,1]$. This was shown earlier in [8], 3.4.2.

THEOREM 4.10. There is a non-maximal prime $z$-filter $\mathscr{F}$ on $[0,1]$, such that, given any rank one set $A$, there exists $F \in \mathscr{F}$ such that $A \cap F \subset\{0\}$

Proof. For $G$ a closed subset of $(0,1]$, we define

$$
\zeta(G)=\lim _{t \rightarrow 0} \frac{\int_{0}^{t} \chi_{G}}{t}
$$

when this limit exists. Observe that the family of sets $\{G \mid \zeta(G)=1\}$ is a $z$-filter, and can be extended to a maximal $z$-filter on $(0,1]$. This induces a prime $z$-filter $\mathscr{F}$ on $[0,1]$. Let $A$ be a set of isolated points of $(0,1]$. There exists a neighborhood of $A, \mathscr{O}_{A}$, such that $\zeta\left(\overline{\mathscr{O}}_{A}\right)=0$. So the complement of $\mathscr{O}_{A}$ is in $\mathscr{F}$.

5. Residue class domains of $C^{\infty}([0,1], \mathbf{R})$. As a byproduct of $\S \S 2$ and 3 we get some results on $C^{\infty}([0,1], \mathbf{R})$. We shall classify 20 residue class domains, 2 for each of the 10 residue class domains of $c$.

5.1. Residue class domains of $C^{\infty}([0,1], \mathbf{R})$ that are inherited from $C([0,1], \mathbf{R})$. In this part of $\S 5$ we classify one residue class domain of $C^{\infty}([0,1], \mathbf{R})$ for every residue class domain of $c$.

For any sequence of points $Y=\left\{y_{n}\right\}$ in $[0,1]$, converging to zero, the restriction map

$$
\text { rest: } C^{\infty}([0,1], \mathbf{R}) \rightarrow C(\bar{Y}, \mathbf{R})
$$

has image isomorphic to a subring of $c$. Further if there is enough space between the $y_{n}$ 's (e.g. $\left.y_{n}=1 / n\right)$, then the image of $\left\{f \mid f^{(k)}(0)=\right.$ 0 , for all $k \geq 0\}$ under rest is a convex ideal of $c$. That is, given any $f: Y \rightarrow \mathbf{R}$ which is eventually less then any power of $x, f$ can be "glued together" to form a $C^{\infty}$ function. Therefore, if $\mathscr{F}$ is a nonmaximal prime $z$-filter on $[0,1]$ and $\bar{Y} \in \mathscr{F}$ then $C^{\infty}([0,1], \mathbf{R}) / p_{\mathscr{F}}$ 
$\left(p_{\mathscr{F}}\right.$ is the set of functions that are zero on an element of $\mathscr{F}$ ) is isomorphic to a subring of $c / p_{\mathscr{U}(\mathscr{F})}(\mathscr{U}(\mathscr{F})$ is the ultrafilter induced on $Y$ by $\mathscr{F}) ; C^{\infty}([0,1], \mathbf{R}) / p_{\mathscr{F}}$ is the vector sum of subring isomorphic to $\mathbf{R}[[x]]$ and a prime ideal whose image is convex in $c / p_{\mathscr{U}}$. Further for any non-maximal prime ideal $p \subset C^{\infty}([0,1], \mathbf{R})$ with associated prime $z$-filter $\mathscr{F}$, if $\bar{Y} \in \mathscr{F}$ then there exists a prime ideal $p \subset c$ such that $C^{\infty}([0,1], \mathbf{R}) / p$ is isomorphic to a subring of $c / p$. In this case $C^{\infty}([0,1], \mathbf{R}) / p$ is the vector sum of a subring isomorphic to $\mathbf{R}[[x]]$ and a prime ideal whose image is convex in $c / p$. Further for two such prime ideals $p_{1}, p_{2} \subset C^{\infty}([0,1], \mathbf{R})$,

$$
C^{\infty}([0,1], \mathbf{R}) / p_{1} \simeq C^{\infty}([0,1], \mathbf{R}) / p_{2} \quad \text { iff } c / p_{1} \simeq c / p_{2} .
$$

Notation 5.1.1.

$$
\begin{aligned}
\mathscr{M}_{y} & =\{f \in C([0,1], \mathbf{R}) \mid f(y)=0\}, \\
Q_{y} & =\left\{f \in C^{\infty}([0,1], \mathbf{R}) \mid f^{(k)}(y)=0 \text { for all } k\right\} .
\end{aligned}
$$

We shall also write $\mathscr{M}$ for $\mathscr{M}_{0}$ and $Q$ for $Q_{0}$. For a prime $z$-filter $\mathscr{F}$,

$$
\begin{aligned}
& p_{\mathscr{F}}=\{f \in C([0,1], \mathbf{R}) \mid Z(f) \in \mathscr{F}\}, \\
& q_{\mathscr{F}}=\left\{f \in C^{\infty}([0,1], \mathbf{R}) \mid \forall k, f^{(k)} \in p_{\mathscr{F}}\right\} .
\end{aligned}
$$

For a prime ideal $p$ of $C([0,1], \mathbf{R}), p^{\#}$ denotes $p \cap C^{\infty}([0,1], \mathbf{R})$.

Fact 5.1.2.

$$
\begin{aligned}
& C^{\infty}([0,1], \mathbf{R}) / \mathscr{M}_{y}^{\#} \simeq \mathbf{R}, \\
& C^{\infty}([0,1], \mathbf{R}) / Q_{y} \simeq \mathbf{R}[[x]] .
\end{aligned}
$$

Notation 5.1.3. For a rank one set $F \subset[0,1]$, with a unique accumulation point, we denote by $\rho_{F}$ and $\rho_{F^{*}}$ :

$$
\begin{gathered}
\rho_{F}: C([0,1], \mathbf{R}) \rightarrow c, \\
\rho_{F^{*}}: C^{\infty}([0,1], \mathbf{R}) \rightarrow c
\end{gathered}
$$

the maps induced by the restriction to the isolated points of $F$.

Observe that $\rho_{F}$ is onto.

Fact 5.1.4. Let $F$ be as in Notation 5.1.3. Let $\mathscr{F}$ be a nonmaximal prime $z$-filter containing $F$. Then $\rho_{F^{*}}\left(p_{\mathscr{F}}^{\#}\right)$ is a prime ideal of $\rho_{F^{*}}\left(C^{\infty}([0,1], \mathbf{R})\right.$. In fact there exists a non-principal ultrafilter $\mathscr{U}$ on $\mathbf{N}$ such that

$$
\rho_{F^{*}}\left(p_{\mathscr{F}}^{\#}\right)=p_{\mathscr{U}} \cap \rho_{F^{*}}\left(C^{\infty}([0,1], \mathbf{R})=\rho_{F}\left(p_{\mathscr{F}}\right) \cap \rho_{F^{*}}\left(C^{\infty}([0,1], \mathbf{R}) .\right.\right.
$$

Notation 5.1.5. For $F$ and $\mathscr{F}$ as in Fact 5.1.4, we denote by $\mathscr{U}_{F}(\mathscr{F})$ (or just $\mathscr{U}(\mathscr{F})$ if no confusion is likely) the non-principal ultrafilter on $\mathbf{N}$ such that $\rho_{F}\left(p_{\mathscr{F}}\right)=p_{\mathscr{U}}$. 
We let $\rho_{F}^{*}$ denote the isomorphism

$$
\rho_{F}^{*}: C([0,1], \mathbf{R}) / p_{\mathscr{F}} \rightarrow c / p_{\mathscr{U}_{F}}(\mathscr{F})
$$

induced by $\rho_{F}$ and we let $\rho_{F}^{(\#)}$ denote the monomorphism

$$
\rho_{F}^{(\#)}: C^{\infty}([0,1], \mathbf{R}) / p_{\mathscr{F}}^{\#} \rightarrow c / p_{\mathscr{U}_{F}(\mathscr{F})}
$$

induced by $\rho_{F^{*}}$.

As we said earlier, if there is enough space between the $x_{n}$ 's in a sequence $\left\{x_{n}\right\}_{n=1}^{\infty}$ and $g$ is a function dominated by all powers of $(x-y)$ then we can $C^{\infty}$ patch together the restriction of $g$ to $F$.

Therefore we define:

Definition 5.1.6. A closed set $F=\left\{x_{n}\right\}_{n=1}^{\infty} \cup\{y\}, F \subset[0,1]$ is a set having polynomial distances if

(i) $\lim _{n \rightarrow \infty} x_{n}=y$.

(ii) $\left|y-x_{n}\right|>\left|y-x_{n+1}\right|$.

(iii) Either for all $n, x_{n}>y$, or for all $n, x_{n}<y$.

(iv) There exist $m \in \mathbf{N}$ and $M \in \mathbf{R}$ such that $M\left|x_{n}-x_{n+1}\right|>$ $\left|x_{n}-y\right|^{m}$ for all $n$.

The most obvious example of such a set is $\{1 / n\}_{n=1}^{\infty} \cup\{0\}$. From now on we assume, without loss of generality, that $y$ is the origin. We shall show that if $\mathscr{F}$ contains a set having polynomial distances, then the image of $C^{\infty}([0,1], \mathbf{R}) / p_{\mathscr{F}}$ can be described as the vector sum of a subring isomorphic to $\mathbf{R}[[x]]$ and a prime ideal which is convex in $l^{\infty} / p_{\mathscr{U}(\mathscr{F})}$. We shall finally show that for two such prime $z$-filters $\mathscr{F}$ and $\mathscr{G}$, the following are equivalent:

(i) $c / p_{\mathscr{U}(\mathscr{F})} \simeq c / p_{\mathscr{U}(\mathscr{G})}$.

(ii) $C^{\infty}([0,1], \mathbf{R}) / p_{\mathscr{F}}^{\#} \simeq C^{\infty}([0,1], \mathbf{R}) / p_{\mathscr{G}}^{\#}$.

(iii) $C^{\infty}([0,1], \mathbf{R}) / q_{\mathscr{F}} \simeq C^{\infty}([0,1], \mathbf{R}) / q_{\mathscr{G}}$.

We also have a similar result for all prime ideals $p$ of $C^{\infty}([0,1], \mathbf{R})$ such that $p_{\mathscr{F}}^{\#} \subset p \not \subset Q$.

We shall need to use bump functions. 
Notation 5.1.7. We let

$$
\begin{aligned}
\varphi(x) & = \begin{cases}e^{-1 / x} \cdot e^{-1 /(1-x)} & \text { if } 0<x<1, \\
0 & \text { if } 1 \leq x \text { or } x \leq 0,\end{cases} \\
\varphi_{r}(x) & = \begin{cases}e^{-r / x} \cdot e^{-r /(r-x)} & \text { if } 0<x<r, \\
0 & \text { if } r \leq x \text { of } x \leq 0,\end{cases} \\
\varphi_{r, b}(x) & = \begin{cases}e^{-r /(x-b)} \cdot e^{-r /[r-(x-b)]} & \text { if } b<x<r+b, \\
0 & \text { if } r+b \leq x \text { or } x \leq b .\end{cases}
\end{aligned}
$$

LEMMA 5.1.8. For $\varphi, \varphi_{r}$, and $\varphi_{r, b}$ as above, for all $b$ and all $r \neq 0$,

$$
\sup \left|\varphi_{r, b}^{(k)}(x)\right|=\sup \left|\varphi_{r}^{(k)}(x)\right|=\left(1 /\left(r^{k}\right)\right) \sup \left|\varphi^{(k)}(x)\right|
$$

for all $k$.

Proof. Left to reader.

As corollaries of Lemma 5.1.8, we get the following lemmas.

LEMMA 5.1.9. Let $F \subset[0,1]$ be such that $F=\left\{x_{n}\right\}_{n=1}^{\infty} \cup\{0\}$ with

(i) $\lim _{n \rightarrow \infty} x_{n}=0$.

(ii) $x_{n}>x_{n+1}$.

Let $\left\{c_{n}\right\}_{n=1}^{\infty}$ be a real valued sequence such that, for all $k \in \mathbf{N}$, there exists a constant $K_{k}$ such that

$$
K_{k} \cdot\left|x_{n}-x_{n+1}\right|^{k}>\left|c_{n}\right|
$$

and

$$
K_{k} \cdot\left|x_{n-1}-x_{n}\right|^{k}>\left|c_{n}\right|
$$

for all $n \in \mathbf{N}$.

Then there exists $g \in C^{\infty}([0,1], \mathbf{R})$ such that $g\left(x_{n}\right)=c_{n}$.

Proof. We define a family of functions $\left\{h_{n}\right\}$ :

$$
h_{n}=e^{4} \cdot \varphi_{r_{n}, b_{n}}
$$

where

$$
\begin{aligned}
& r_{n}=\min \left(\left|x_{n}-x_{n+1}\right|,\left|x_{n}-x_{n-1}\right|\right) \\
& b_{n}=x_{n}-(1 / 2) r_{n}
\end{aligned}
$$


Let

$$
g(x)=\sum_{n=1}^{\infty} c_{n} \cdot h_{n}(x)
$$

It can be checked that $g$ is $C^{\infty}$.

LEMMA 5.1.10. Let $F \subset[0,1]$ be a compact rank 1 set. Let $F=$ $\left\{x_{n}\right\}_{n=1}^{\infty} \cup\{0\}$. Let $F$ have polynomial distances. Let $\mathscr{F}$ be a nonmaximal prime $z$-filter on $[0,1]$ such that $F \in \mathscr{F}$. Let $\left\{f_{j}\right\}_{j=0}^{\infty}$ be a family of $C^{\infty}([0,1], \mathbf{R})$ functions such that $f_{j}^{(k)}(0)=0$ for all $j \in \mathbf{N} \cup\{0\}$ and all $k \in \mathbf{N} \cup\{0\}$. Then there exists $f \in C^{\infty}([0,1], \mathbf{R})$ such that for all $j \in \mathbf{N} \cup\{0\}, f^{(j)} \equiv \mathscr{F} f_{j}$.

Proof. Let $M \in \mathbf{R}$ and $m \in \mathbf{N}$ be such that

$$
M \cdot\left|x_{n}-x_{n+1}\right|>x_{n}^{m} \text { for all } n \in \mathbf{N} \text {. }
$$

We put an additional condition on $F$, that $\left(x_{n}\right) /\left(x_{n+1}\right)$ is bounded. (If necessary, we can always add points.)

We shall prove this lemma by constructing Taylor polynomials at each $x_{n}$, and then patching them together with bump functions. Let $r_{n}=x_{n}-x_{n+1}, b_{n}=x_{n+1}$. We define

$$
\begin{gathered}
\zeta_{0}=0, \\
\zeta_{n}(x)=\left(\int_{x_{n+1}}^{x} \varphi_{r_{n}, b_{n}}(t) d t\right)\left(\int_{x_{n+1}}^{x_{n}} \varphi_{r_{n}, b_{n}}(t) d t\right)^{-1} \text { for all } n \geq 1 .
\end{gathered}
$$

Observe that $\zeta_{n}\left(x_{n}\right)=1, \zeta_{n}^{(k)}\left(x_{n}\right)=0$ for all $k \geq 1$. The problem now is to choose a suitable degree $d(n)$, for the Taylor polynomial at $x_{n}$ so that

$$
f(x)=\sum_{n=1}^{\infty}\left(\left[\zeta_{n}(x)-\zeta_{n-1}(x)\right] \sum_{j=0}^{d(n)} f_{j}\left(x_{n}\right)\left(x-x_{n}\right)^{j} /(j !)\right)
$$

is a $C^{\infty}([0,1], \mathbf{R})$ function.

We observe that for all $j \in \mathbf{N}$ and $k \in \mathbf{N}$, there exists $K_{j, k} \in \mathbf{R}$ such that

$$
f_{j}(x) \leq K_{j, k} x^{k} \quad \text { for all } x
$$


For $x, x_{n}<x<x_{n-1}$, we have

$$
\begin{aligned}
\left|f^{(k)}(x)\right|= & \mid\left[\zeta_{n-1}(x)\left(\sum_{j=0}^{d(n-1)} f_{j}\left(x-x_{n-1}\right)^{j} /(j !)\right)\right. \\
& \left.+\left(1-\zeta_{n-1}(x)\right)\left(\sum_{j=0}^{d(n)} f_{j}\left(x_{n}\right)\left(x-x_{n}\right)^{j} / j\right)\right]^{(k)} \mid \\
\leq & \left(\frac{2^{k}}{\left(x_{n-1}-x_{n}\right)^{k}}+1\right)\left(\sum_{j=0}^{d(n-1)}\left|f_{j}\left(x_{n-1}\right)\right|+\sum_{j=0}^{d(n)}\left|f_{j}\left(x_{n}\right)\right|\right) \\
\leq & \frac{2^{k+1} M^{k}}{\left(x_{n-1}\right)^{m k}}\left(2 \sum_{j=0}^{d(n)} K_{j, k m_{2}} x_{n-1}^{k m+2}\right) \\
\leq & 2^{k+2} M^{k} x_{n-1}^{2}\left(\sum_{j=0}^{d(n)} K_{j, k m+2}\right) .
\end{aligned}
$$

So we define $d(n)$ :

$$
\begin{gathered}
d(1)=1, \\
d(n)=\max \left\{s \mid \sum_{j=0}^{s} K_{j, k m+2} 2^{k+2} M^{k} x_{n-1}<1, \text { for all } k \leq s\right\}
\end{gathered}
$$

or

$$
d(n)=1 \text { if the set of such s's is empty. }
$$

Observe that since $M$ and $K_{j, k m+2}$ do not depend directly on $n$,

$$
\lim _{n \rightarrow \infty} d(n)=\infty \text {. }
$$

Observe also that since $\left(x_{n-1}\right) /\left(x_{n}\right)$ is bounded, $f^{(k)}$ is now dominated by $x$. The proof is complete.

Lemma 5.1 .10 will mainly be useful for studying $C^{\infty}([0,1], \mathbf{R}) / q_{\mathscr{F}}$. We have the following proposition as a result of Lemma 5.1.9. We earlier said that under certain conditions $C^{\infty}([0,1], \mathbf{R}) / p_{\mathscr{F}}^{\#}$ could be described as a vector sum of a subring isomorphic to $\mathbf{R}[[x]]$ and a prime ideal whose image is convex in $c / p_{\mathscr{U}(\mathscr{F})}$. Our next proposition will essentially prove this. We also show that this prime ideal is $\omega_{1}$ saturated. As a byproduct we get a result for all non-maximal prime $z$-filters that contain rank one sets. 
Proposition 5.1.11. Let $F$ be a rank one set with a unique limit point. Let $\mathscr{F}$ be a non-maximal prime $z$-filter with $F \in \mathscr{F}$. Then

(a) $\left.\rho_{F}^{(\#)}\left(C^{\infty}[0,1], \mathbf{R}\right) / p_{\mathscr{F}}^{\#}\right)$ contains a convex prime ideal of $c / p_{\mathscr{U}(\mathscr{F})}$. Further, if $F$ has polynomial distances, then (letting $y$ be the limit point of $F$ )

(b) $\rho_{F}^{(\#)}\left(Q_{y} / p_{\mathscr{F}}^{\#}\right)$ is a convex prime ideal of $c / p_{\mathscr{U}(\mathscr{F})}$.

(c) $Q_{y} / p_{\mathscr{F}}^{\#}$ has no countable cofinal subset.

Proof. (a) and (b) follow immediately from Lemma 5.1.9. To prove (c), we let $\left\{f_{j}\right\}_{j=1}^{\infty}$ be a countable set of sequences such that

(i) for all $n \in \mathbf{N}, f_{j+1}\left(x_{n}\right) \geq f_{j}\left(x_{n}\right)>0$ and

(ii) for each $j \in \mathbf{N}$ and $k \in \mathbf{N}$, there exists a constant $K_{j k}$ such that $K_{j k}\left(x_{n}\right)^{k}>f_{j}\left(x_{n}\right)$ for all $n$.

We now construct a function $f$ such that $f>_{\mathscr{F}} f_{j}$ for all $j \in$ $\mathbf{N}$ and such that, for all $k \in \mathbf{N}$, there exists a constant $K_{k}$ with $K_{k}\left(x_{n}\right)^{k}>\left|f\left(x_{n}\right)\right|$. This will prove the proposition.

To construct $f$, we first define $d(n)$ :

$$
d(n)=\max \left\{k \leq n \mid K_{k k} \cdot\left(x_{n}\right) \leq 1\right\} .
$$

Then

$$
f\left(x_{n}\right)=f_{1}\left(x_{n}\right) \vee \cdots \vee f_{d(n)}\left(x_{n}\right) .
$$

This completes the proof.

From here on, $\mathscr{F}$ and $\mathscr{G}$ will denote non-maximal prime $z$-filters with rank one sets $F \in \mathscr{F}$ and $G \in \mathscr{G}$, each having polynomial distances, each having the origin as their sole limit point.

We now wish to study how the different types of ultrafilters, $\mathscr{U}(\mathscr{F})$, affect $C^{\infty}([0,1], \mathbf{R}) / p_{\mathscr{F}}^{\#}$. In studying $c / p$, the ultrafilter affected the set $\left(l^{\infty} / p-c / p\right)$. In the current case, it affects the set of those functions which are less $(\bmod \mathscr{F})$ than all powers of $x$ and greater $(\bmod \mathscr{F})$ than all flat at the origin $C^{\infty}$ functions.

Lemma 5.1.12. Let $\mathscr{U}(\mathscr{F})$ be a P-point. Let $f \in C([0,1], \mathbf{R})$ be such that

$$
|f|<\mathscr{F} x^{k} \quad \text { for all } k \in \mathbf{N} \text {. }
$$

Then there exists $g \in C^{\infty}([0,1], \mathbf{R})$ such that $f \equiv \mathscr{F} g$.

Proof. Consider the following subsets of $\left\{x_{n}\right\}_{n=1}^{\infty}$,

$$
A_{j}=\left\{x_{n}|| f\left(x_{n}\right) \mid<x_{n}^{j}\right\} \text {. }
$$


Notice that $A_{j} \in \mathscr{U}(\mathscr{F})$ for all $j \in \mathbf{N}$. Since $\mathscr{U}(\mathscr{F})$ is a $P$-point there exists $A \in \mathscr{U}(\mathscr{F})$ such that $\left(A-A_{j}\right)$ is finite for all $j \in \mathbf{N}$. Hence, for each $j \in \mathbf{N}$, there exists a constant $K_{j}$ such that

$$
\left|f\left(x_{n}\right)\right|<K_{j} x_{n}^{j} \text { for all } x_{n} \in A .
$$

The result follows from Lemma 5.1.9.

LeMMA 5.1.13. Let $\mathscr{U}(\mathscr{F})$ be a non-P-point. Then

(a) There exists $f \in C([0,1], \mathbf{R})$ such that $|f|<\mathscr{F} x^{j}$ for all $j \in \mathbf{N}$ and $f>_{\mathscr{F}}|g|$ for all $g \in C^{\infty}([0,1], \mathbf{R})$ such that $g^{(k)}(0)=0$ for all $k \in \mathbf{N}$.

(b) The set of all $f$ as in (a) does not have a countable cofinal subset.

(c) The set of all $f$ as in (a) has a countable coinitial subset if and only if $\left(l_{0, \mathscr{U}(\mathscr{F})} / c_{0, \mathscr{U}(\mathscr{F})}\right)_{+}$does.

Proof. We shall prove all three at once. We first observe that the families $\left\{\left\{a_{n}\right\} \mid a_{n}=1 /(k(n))\right\}$ and $\left\{h \mid h\left(x_{n}\right)=x_{n}^{k(n)}\right\}$ where $k(n) \in$ $\mathbf{N}$, are cofinal and coinitial in their respective sets. We have the obvious map $\{1 /(k(n))\} \rightarrow\left\{x_{n}^{k(n)}\right\}$ which clearly preserves order and "non-convergence." The result follows immediately.

We shall now let $c / p_{\mathscr{U}(\mathscr{F})} \simeq c / p_{\mathscr{U}(\mathscr{G})}$ and use Ax-Kochen machinery to build up an isomorphism from $\operatorname{Frac}\left(C^{\infty}([0,1], \mathbf{R}) / p_{\mathscr{F}}^{\#}\right)$ to $\operatorname{Frac}\left(C^{\infty}([0,1], \mathbf{R}) / p_{\mathscr{G}}^{\#}\right)$ which sends $C^{\infty}([0,1], \mathbf{R}) / p_{\mathscr{F}}^{\#}$ to $C^{\infty}([0,1], \mathbf{R}) / p_{\mathscr{G}}^{\#}$. We recall that $Q / p_{\mathscr{F}}$ and $Q / p_{\mathscr{G}}$ are convex in $C([0,1], \mathbf{R}) / p_{\mathscr{F}}, \operatorname{so} \operatorname{Frac}\left(C^{\infty}([0,1], \mathbf{R}) / p_{\mathscr{F}}^{\#}\right)=\operatorname{Frac}\left(C([0,1], \mathbf{R}) / p_{\mathscr{F}}\right)$.

Recall that, by 1.35 , a valued field $(K, R, I, G, v, \bar{K})$ can be determined up to isomorphism by the pair $(K, I)$. We shall be denoting valued fields by these pairs (as we did before in $\S 2$ ).

Notation 5.1.14. The bounded continuous functions on $(0,1]$ will be denoted $C^{*}((0,1], \mathbf{R})$.

Proposition 5.1.15. For $\mathscr{F}$ and $\mathscr{G}$ as above let

$$
\begin{aligned}
& r_{\mathscr{F}}=\left\{\left.f \in C([0,1], \mathbf{R})|| f\right|_{\mathscr{F}} x^{k}, \forall k \in \mathbf{N}\right\}, \\
& r_{\mathscr{G}}=\left\{\left.f \in C([0,1], \mathbf{R})|| f\right|_{\mathscr{G}} x^{k}, \forall k \in \mathbf{N}\right\} .
\end{aligned}
$$

Then there is an isomorphism

$$
\psi: \operatorname{Frac}\left(C([0,1], \mathbf{R}) / r_{\mathscr{F}}\right) \rightarrow \operatorname{Frac}\left(C([0,1], \mathbf{R}) / r_{\mathscr{G}}\right)
$$


such that

(a) $\psi\left(C^{\infty}([0,1], \mathbf{R}) / r_{\mathscr{F}}^{\#}\right)=C^{\infty}([0,1], \mathbf{R}) / r_{\mathscr{G}}^{\#}$ and

(b) For $f, g \in C^{\infty}([0,1], \mathbf{R})$, if $\psi\left(\left[f+r_{\mathscr{F}}\right]\right)=\left[g+r_{\mathscr{G}}\right]$ then $f^{(k)}(0)=g^{(k)}(0)$ for all $k \in \mathbf{N}$.

Proof. Let

$$
s_{\mathscr{F}}=\left\{\left.f|\exists k \in \mathbf{N},| f\right|^{k} \underset{\mathscr{F}}{<x}\right\} .
$$

Define $s_{\mathscr{G}}$ similarly.

Consider the valued fields

$$
\begin{array}{cc}
\left(K=\operatorname{Frac}\left(C([0,1], \mathbf{R}) / s_{\mathscr{F}}\right),\right. & \left.R=C^{*}((0,1], \mathbf{R}) / s_{\mathscr{F}}\right) \quad \text { and } \\
\left(K=\operatorname{Frac}\left(C([0,1], \mathbf{R}) / s_{\mathscr{G}}\right),\right. & \left.R=C^{*}((0,1], \mathbf{R}) / s_{\mathscr{G}}\right) .
\end{array}
$$

(By 1.33 these pairs also describe the valued fields up to isomorphism.) These are real closed valued fields with convex valuations. The residue class fields are both $\mathbf{R}$. The valuation groups are both $\omega_{1}$-saturated. $\omega$-pseudo-completeness follows from 2.1.22. Therefore, we have an isomorphism

$$
\psi_{1}: \operatorname{Frac}\left(C([0,1], \mathbf{R}) / s_{\mathscr{F}}\right) \rightarrow \operatorname{Frac}\left(C([0,1], \mathbf{R}) / s_{\mathscr{G}}\right)
$$

such that for all constant functions $b$,

$$
\psi_{1}\left(\left[b+s_{\mathscr{F}}\right]\right)=\left[b+s_{\mathscr{G}}\right] .
$$

Consider the real closed fields with convex valuation

$$
\begin{aligned}
& \left(K=\operatorname{Frac}\left(C([0,1], \mathbf{R}) / r_{\mathscr{F}}\right), I=s_{\mathscr{F}} / r_{\mathscr{F}}\right) \quad \text { and } \\
& \left.\left(K=\operatorname{Frac}([0,1], \mathbf{R}) / r_{\mathscr{G}}\right), I=s_{\mathscr{G}} / r_{\mathscr{G}}\right) .
\end{aligned}
$$

In both cases,

(i) The valuation group is $\mathbf{R}$.

(ii) There is a cross section $\pi(\alpha)=x^{\alpha}$.

(iii) The valued field is $\omega$-pseudo-complete by 2.1.22.

(iv) The residue class field is

$$
\operatorname{Frac}\left(C([0,1], \mathbf{R}) / s_{\mathscr{F}}\right) \quad\left(\operatorname{or} \operatorname{Frac}\left(C([0,1], \mathbf{R}) / s_{\mathscr{G}}\right)\right) \text {. }
$$

Therefore, we have an isomorphism $\psi$ such that for a polynomial $g$,

$$
\psi\left(\left[g+r_{\mathscr{F}}\right]\right)=\left[g+r_{\mathscr{G}}\right] .
$$

Let $f \in C^{\infty}([0,1], \mathbf{R})$ be such that $f^{(k)}(0)=a_{k}$. Then $[f]$ is the unique pseudo-limit of the sequence

$$
\left\{\left[\sum_{0}^{n} a_{k} x^{k} /(k !)\right]\right\} \text {. }
$$


Hence

$$
\psi\left(\left[f+r_{\mathscr{F}}\right]\right)=\left[f+r_{\mathscr{G}}\right] .
$$

Therefore, $\psi\left(\left[f+r_{\mathscr{F}}\right]\right)=\left[g+r_{\mathscr{G}}\right]$ implies $(f-g) \in r_{\mathscr{G}}$ and hence $(f-g)^{(k)}(0)=0$ for all $k$ (assuming $g$ is $C^{\infty}$ ).

It is interesting that Proposition 5.1.15 does not depend on the ultrafilters $\mathscr{U}(\mathscr{F})$ and $\mathscr{U}(\mathscr{G})$. Further, it does not matter at all which power of $x$ is dominated by the distances between $x_{n}$ and $x_{n+1}$; the analysis in this paper does not distinguish between $\left\{(1 / 2)^{n}\right\}_{n=1}^{\infty} \cup\{0\}$ and $\{1 / \sqrt{n}\}_{n=1}^{\infty} \cup\{0\}$.

Notation 5.1.16.

$$
\begin{aligned}
& t_{\mathscr{F}}=\{f \in C([0,1], \mathbf{R}) \mid \exists g \in C([0,1], \mathbf{R}), g \underset{\bar{F}}{\equiv} f \text { and } \\
& \left.\qquad \forall k \in \mathbf{N} \exists M_{k} \in \mathbf{R} ;|g(x)| \leq M_{k} x^{k} \text { for all } x \in[0,1]\right\}
\end{aligned}
$$

$t_{\mathscr{F}}^{\#}$ of course is $Q$.

We next have one of the main theorems of the section.

THEOREM 5.1.17. Let $\mathscr{F}$ and $\mathscr{G}$ be as in Notation 5.1.11. Let $u_{\mathscr{F}}$ and $u_{\mathscr{G}}$ be prime ideals of $C([0,1], \mathbf{R})$ such that $p_{\mathscr{F}} \subset u_{\mathscr{F}} \nsubseteq t_{\mathscr{F}}$ and $p_{\mathscr{G}} \subset u_{\mathscr{G}} \nsubseteq t_{\mathscr{G}}$.

Then the following are equivalent:

(a) $C([0,1], \mathbf{R}) / u_{\mathscr{F}} \simeq C([0,1], \mathbf{R}) / u_{\mathscr{G}}$.

(b) There exists an isomorphism

$$
\psi: C^{\infty}([0,1], \mathbf{R}) / u_{\mathscr{F}}^{\#} \rightarrow C^{\infty}([0,1], \mathbf{R}) / u_{\mathscr{G}}^{\#}
$$

such that if $\psi\left(\left[f+u_{\mathscr{F}}^{\#}\right]\right)=\left[g+u_{\mathscr{G}}^{\#}\right]$ then $f^{(k)}(0)=g^{(k)}(0)$ for all $k \in \mathbf{N}$.

In particular, the following are equivalent

(a1) $C([0,1], \mathbf{R}) / p_{\mathscr{F}} \simeq C([0,1], \mathbf{R}) / p_{\mathscr{G}}$.

(b1) There exists an isomorphism

$$
\theta: C^{\infty}([0,1], \mathbf{R}) / p_{\mathscr{F}}^{\#} \rightarrow C^{\infty}([0,1], \mathbf{R}) / p_{\mathscr{G}}^{\#}
$$

such that if $\theta\left(\left[f+p_{\mathscr{F}}^{\#}\right]\right)=\left[g+p_{\mathscr{G}}^{\#}\right]$ then $f^{(k)}(0)=g^{(k)}(0)$ for all $k \in \mathbf{N}$.

Proof. (a) $\rightarrow$ (b). By Proposition 5.1.15, (a) implies the existence of an isomorphism

$$
\psi_{2}: \operatorname{Frac}\left(C([0,1], \mathbf{R}) / r_{\mathscr{F}}\right) \rightarrow \operatorname{Frac}\left(C([0,1], \mathbf{R}) / r_{\mathscr{G}}\right)
$$

such that

$$
\psi_{2}\left(C^{\infty}([0,1], \mathbf{R}) / r_{\mathscr{F}}^{\#}\right)=C^{\infty}([0,1], \mathbf{R}) / r_{\mathscr{G}}^{\#}
$$


and for $f \in C^{\infty}([0,1], \mathbf{R})$

$$
\psi_{2}\left(\left[f+r_{\mathscr{F}}\right]\right)=\left[f+r_{\mathscr{G}}\right] .
$$

By Lemma 5.1.13, the valuation group of the valued field

$$
\left(K=\operatorname{Frac}\left(C([0,1], \mathbf{R}) / t_{\mathscr{F}}\right), I=r_{\mathscr{F}} / t_{\mathscr{F}}\right)
$$

is isomorphic to the valuation group of the valued field

$$
\left(K=\operatorname{Frac}\left(l^{\infty} / c_{0, \mathscr{U}_{F}(\mathscr{F})}\right), I=l_{0, \mathscr{U}_{F}(\mathscr{F})} / c_{0, \mathscr{U}_{F}(\mathscr{F})}\right) .
$$

But by (a) this is isomorphic to the valuation group of the valued field

$$
\left(K=\operatorname{Frac}\left(l^{\infty} / c_{0, \mathscr{U}_{G}(\mathscr{G})}\right), I=l_{0, \mathscr{U}_{G}(\mathscr{G})} / c_{0, \mathscr{U}_{G}(\mathscr{G})}\right),
$$

which is isomorphic to the valuation group of the valued field

$$
\left.\left(K=\operatorname{Frac}(C[0,1], \mathbf{R}) / t_{\mathscr{G}}\right), I=r_{\mathscr{G}} / t_{\mathscr{G}}\right) .
$$

Hence we have an analytic isomorphism

$$
\begin{aligned}
&\left(\psi_{3}, \psi_{3}^{\#}\right):(K=\operatorname{Frac}(C( {\left.\left.[0,1], \mathbf{R}) / t_{\mathscr{F}}\right), I=r_{\mathscr{F}} / t_{\mathscr{F}}\right) } \\
&\left(K=\operatorname{Frac}\left(C([0,1], \mathbf{R}) / t_{\mathscr{G}}\right), I=r_{\mathscr{G}} / t_{\mathscr{G}}\right) .
\end{aligned}
$$

Since $r_{\mathscr{F}}^{\#} \subset t_{\mathscr{F}}$ and $r_{\mathscr{G}}^{\#} \subset t_{\mathscr{G}}$, the valuations are trivial on

$$
C^{\infty}([0,1], \mathbf{R}) / t_{\mathscr{F}}^{\#} \quad \text { and } \quad C^{\infty}([0,1], \mathbf{R}) / t_{\mathscr{G}}^{\#}
$$

therefore we can require that for all $f \in C^{\infty}([0,1], \mathbf{R})$,

$$
\psi_{3}\left(\left[f+t_{\mathscr{F}}\right]\right)=\left[f+t_{\mathscr{G}}\right] .
$$

We do this by choosing the initial subfields $K_{0, \mathscr{F}}$ and $K_{0, \mathscr{G}}$ such that

$$
\left[f+t_{\mathscr{F}}\right] \in K_{0, \mathscr{F}} \quad \text { and }\left[f+t_{\mathscr{G}}\right] \in K_{0, \mathscr{G}}
$$

for all $f \in C^{\infty}([0,1], \mathbf{R})$. Then letting

$$
\operatorname{residue}_{0, \mathscr{F}}: K_{0, \mathscr{F}} \rightarrow \operatorname{Frac}\left(C([0,1], \mathbf{R}) / r_{\mathscr{F}}\right)
$$

and

$$
\text { residue }_{0, \mathscr{G}}: K_{0, \mathscr{G}} \rightarrow \operatorname{Frac}\left(C([0,1], \mathbf{R}) / r_{\mathscr{G}}\right)
$$

be the restrictions of the residue maps, clearly

$$
\left(\text { residue }_{0, \mathscr{G}}\right)^{-1} \circ \psi_{2} \circ\left(\text { residue }_{0, \mathscr{F}}\right)\left(\left[f+t_{\mathscr{F}}\right]\right)=\left[f+t_{\mathscr{G}}\right],
$$

and $\psi_{3}$ is an extension of

$$
\left(\text { residue }_{0, \mathscr{G}}\right)^{-1} \circ \psi_{2} \circ\left(\text { residue }_{0, \mathscr{F}}\right) \text {. }
$$

Finally we consider the valued field

$$
\left(K=\operatorname{Frac}\left(C([0,1], \mathbf{R}) / u_{\mathscr{F}}\right), I=\left(t_{\mathscr{F}} / u_{\mathscr{F}}\right)\right) .
$$


Claim. $t_{\mathscr{F}} / u_{\mathscr{F}}$ does not have a countable cofinal subset (nor does $\left.t_{\mathscr{G}} / u_{\mathscr{G}}\right)$.

Proof of Claim. Observe that the families

$$
\begin{gathered}
\left\{\left\{a_{n}\right\} \mid a_{n}=1 /(k(n))\right\}_{k \in \mathbf{N}^{\mathbf{N}}} \subset l^{\infty}, \\
\left\{h \mid h\left(x_{n}\right)=x_{n}^{k(n)}\right\}_{k \in \mathbf{N}} \subset C([0,1], \mathbf{R})
\end{gathered}
$$

are cofinal in $c_{0, \mathscr{U}(\mathscr{F})} / p_{\mathscr{U}(\mathscr{F})}$ and $t_{\mathscr{F}} / u_{\mathscr{F}}$ respectively. As in Lemma 5.1 .13 , the map

$$
\{1 /(k(n))\} \rightarrow\left\{x^{k(n)}\right\}
$$

preserves order and "convergence." $c_{0, \mathscr{U}(\mathscr{F})} / p_{\mathscr{U}(\mathscr{F})}$ has no countable cofinal subset, neither does $\left(t_{\mathscr{F}} / u_{\mathscr{F}}\right)$.

This claim shows that the valuation group of

$$
\left(K=\operatorname{Frac}\left(C([0,1], \mathbf{R}) / u_{\mathscr{F}}\right), I=\left(t_{\mathscr{F}} / u_{\mathscr{F}}\right)\right)
$$

is a convex subgroup of an $\omega_{1}$-saturated Abelian group (similarly for $t_{\mathscr{G}}$ and $u_{\mathscr{G}}$ ).

(a) clearly forces the two valuation groups to be the same type of convex subgroup, hence isomorphic. So we have an isomorphism

$$
\psi: \operatorname{Frac}\left(C([0,1], \mathbf{R}) / u_{\mathscr{F}}\right) \rightarrow \operatorname{Frac}\left(C([0,1], \mathbf{R}) / u_{\mathscr{G}}\right)
$$

with

$$
\psi\left(t_{\mathscr{F}} / u_{\mathscr{F}}\right)=t_{\mathscr{G}} / u_{\mathscr{G}}
$$

Since by Lemma $5.1 .9, t_{\mathscr{F}} / u_{\mathscr{F}}=\left(t_{\mathscr{F}}^{\#} / u_{\mathscr{F}}^{\#}\right)$ and $t_{\mathscr{G}} / u_{\mathscr{G}}=\left(t_{\mathscr{G}}^{\#} / u_{\mathscr{G}}^{\#}\right)$, we have that

$$
\psi\left(C^{\infty}([0,1], \mathbf{R}) / u_{\mathscr{F}}^{\#}\right)=C^{\infty}([0,1], \mathbf{R}) / u_{\mathscr{G}}^{\#} .
$$

Let $f, g \in C^{\infty}([0,1], \mathbf{R})$ be such that $\psi\left(\left[f+u_{\mathscr{F}}^{\#}\right]\right)=\left[g+u_{\mathscr{G}}^{\#}\right]$, then $\psi_{3}\left(\left[f+t_{\mathscr{F}}^{\#}\right]\right)=\left[g+t_{\mathscr{G}}^{\#}\right]$. But $\psi_{3}\left(\left[f+t_{\mathscr{F}}^{\#}\right]\right)=\left[f+t_{\mathscr{G}}^{\#}\right]$ so $(f-g) \in t_{\mathscr{G}}^{\#}$ and $f^{(k)}(0)=g^{(k)}(0)$ for all $k \in \mathbf{N}$.

(b) $\rightarrow$ (a). This follows almost immediately from Lemma 5.1.13. Observe that the functions mentioned in Lemma 5.1.13 affect the ring $C^{\infty}([0,1], \mathbf{R}) / u_{\mathscr{F}}^{\#}$ itself. That is, if $f \in r_{\mathscr{F}}-t_{\mathscr{F}}$, then given $g \in t_{\mathscr{F}}^{\#}$, there exists $h \in C^{\infty}([0,1], \mathbf{R})$ such that

$$
\begin{aligned}
& g f \underset{\overline{\mathscr{F}}}{\equiv}, \\
& |g| x^{k} \underset{\mathscr{F}}{ }|h| \text { for all } k \in \mathbf{N}
\end{aligned}
$$

but there does not exist $[\xi] \in C^{\infty}([01],, \mathbf{R}) / u_{\mathscr{F}}^{\#}$ such that $[g] \cdot[\xi]=$ [h]. 
5.2. Residue class domains of $C^{\infty}([0,1], \mathbf{R})$ that inherit the derivation from $C^{\infty}([0,1], \mathbf{R})$. In 5.1 we classified 1 residue class domain of $C^{\infty}([0,1], \mathbf{R})$ for each residue class domain of $c$. In this part of $\S 5$ we shall classify a second residue class domain of $C^{\infty}([0,1], \mathbf{R})$ for each residue class domain of $c$. We are concerned in this part of $\S 5$ with differentially closed prime ideals of $C^{\infty}([0,1], \mathbf{R})$. In particular, if $u$ is a prime ideal as in 5.1 , then we examine

$$
w=\left\{f \mid f^{(k)} \in u, \forall k \geq 0\right\} .
$$

We shall show that

$(\alpha) C^{\infty}([0,1], \mathbf{R}) / u_{1} \simeq C^{\infty}([0,1], \mathbf{R}) / u_{2}$ iff

( $\beta) \quad C^{\infty}([0,1], \mathbf{R}) / w_{1} \simeq C^{\infty}([0,1], \mathbf{R}) / w_{2}$.

Therefore for each residue class domain in 5.1 we get one additional residue class domain. We cannot use the Ax-Kochen machinery to do this, because the valuation ideal would not be contained in $C^{\infty}([0,1], \mathbf{R}) / w$. Instead, we consider the elements of $C^{\infty}([0,1], \mathbf{R}) / w_{i}$ as a sequence of congruence classes

$$
\left\{\left[f_{k}+u_{i}\right]\right\}_{k=0}^{\infty},
$$

where $\left[f_{k}+u_{i}\right]$ is the $k$ th derivative of $\left[f_{0}+u_{i}\right]$. The set of such sequences forms a differential ring. The isomorphism $\xi$ from $C^{\infty}([0,1], \mathbf{R}) / u_{1}$ to $C^{\infty}([0,1], \mathbf{R}) / u_{2}$ induces an isomorphism between the sets of such sequences

$$
\left\{\left[f_{k}+u_{1}\right]\right\} \rightarrow\left\{\xi\left(\left[f_{k}+u_{1}\right]\right)\right\} .
$$

We shall show that under this isomorphism the image of $C^{\infty}([0,1], \mathbf{R}) / w_{1}$ is $C^{\infty}([0,1], \mathbf{R}) / w_{2}$. Astonishingly, it is more work to show that $(\beta)$ implies $(\alpha)$ than vice versa. Of course, if $u_{2} / w_{2}$ is the image of $u_{1} / w_{1}$ then this follows immediately, but this is not always the case. However, it is the case unless $\left(C^{\infty}([0,1], \mathbf{R}) / u_{i}\right)_{+}$ has a coinitial subset of the form $\left\{\left[f+u_{i}\right]^{k}\right\}_{k=1}^{\infty}$. We dispose of this case by showing that if $u_{2} / w_{2}$ is not the image of $u_{1} / w_{1}$ then the radical of the sum of the ideals $u_{2} / w_{2}+\operatorname{Im}\left(u_{1} / w_{1}\right)$ is the smallest prime ideal containing the coinitial set $\left\{\left[f+u_{2}\right]^{k}\right\}_{k=1}^{\infty}$. A similar result holds for $u_{1} / w_{1}+$ preimage $\left(u_{2} / w_{2}\right)$.

Finally we show that for such $w$ there exists a prime ideal $p$, $w \subset p \subset C^{\infty}([0,1], \mathbf{R})$, such that $C^{\infty}([0,1], \mathbf{R}) / p$ is not one of the residue class domains that we classified. In fact we show that there exists a prime ideal $p \subset C^{\infty}([0,1], \mathbf{C})$ such that $p$ is not its own complex conjugate. 
Notation 5.2.1. Let $u_{\mathscr{F}}$ be a prime ideal of $C([0,1], \mathbf{R})$, such that

$$
p_{\mathscr{F}} \subset u_{\mathscr{F}} \nsubseteq t_{\mathscr{F}}
$$

where $p_{\mathscr{F}}$ and $t_{\mathscr{F}}$ are as in 5.1. Then

$$
w_{\mathscr{F}}(u)=\left\{f \in C^{\infty}([0,1], \mathbf{R}) \mid \forall k, f^{(k)} \in u_{\mathscr{F}}^{\#}\right\},
$$

(where $u_{\mathscr{F}}^{\#}=u_{\mathscr{F}} \cap C^{\infty}([0,1], \mathbf{R})$ ).

We shall write $w_{\mathscr{F}}$ unless confusion is likely.

Now we have the main result of this section which shall be proved later.

THEOREM 5.2.2. Let $F$ and $G$ be rank one subsets of $[0,1]$, each with exactly one limit point. Let their least elements be $y_{F}$ and $y_{G}$ respectively. Let $F$ and $G$ have polynomial distances. Let $\mathscr{F}$ and $\mathscr{G}$ be non-maximal prime $z$-filters on $[0,1]$ such that $F \in \mathscr{F}$ and $G \in \mathscr{G}$. Let $u_{\mathscr{F}}$ and $u_{\mathscr{G}}$ be prime ideals of $C([0,1], \mathbf{R})$ such that:

(1) $Z(f) \in \mathscr{F}$ implies $f \in u_{\mathscr{F}}$.

(2) $f \in u_{\mathscr{F}}^{\#}=u_{\mathscr{F}} \cap C^{\infty}\left([0,1]\right.$, R) implies $f^{(k)}\left(y_{F}\right)=0$ for all $k \geq 0$.

(3) There exists $f \in C^{\infty}([0,1], \mathbf{R})$ such that $f \notin u_{\mathscr{F}}$ but $f^{(k)}\left(y_{F}\right)$ $=0$ for all $k \geq 0$.

Let $u_{G}$ have the corresponding properties.

Then the following are equivalent:

(i) $C([0,1], \mathbf{R}) / u_{\mathscr{F}} \simeq C([0,1], \mathbf{R}) / u_{\mathscr{G}}$.

(ii) There exists an isomorphism

$$
\psi: C^{\infty}([0,1], \mathbf{R}) / u_{\mathscr{F}}^{\#} \rightarrow C^{\infty}([0,1], \mathbf{R}) / u_{\mathscr{G}}^{\#}
$$

such that $\psi\left(\left[f+u_{\mathscr{F}}^{\#}\right]\right)=\left[g+u_{\mathscr{G}}^{\#}\right]$ implies $f^{(k)}\left(y_{F}\right)=g^{(k)}\left(y_{G}\right)$ for all $k \geq 0$.

(iii) There exists an isomorphism

$$
\xi: C^{\infty}([0,1], \mathbf{R}) / w_{\mathscr{F}} \rightarrow C^{\infty}([0,1], \mathbf{R}) / w_{\mathscr{G}}
$$

(where $w_{\mathscr{F}}=\left\{f \mid f^{(k)} \in u_{\mathscr{F}}^{\#}\right.$ for all $\left.\left.k \geq 0\right\}\right)$, such that $\xi(D([f]))=$ $D^{\prime}(\xi([f]))$.

Before going further we observe:

First, the theorem would hold if $y_{F}$ and $y_{G}$ were both the greatest elements of $F$ and $G$. If one were greatest and one were least, we should change (ii) to read

$$
f^{(k)}\left(y_{F}\right)=(-1)^{k} g^{(k)}\left(y_{G}\right) .
$$


Second, without loss of generality we can assume $y_{F}=y_{G}=0$.

Third, (i) $\leftrightarrow$ (ii) is 5.1.17. So it remains to show that (ii) $\leftrightarrow$ (iii).

Our first inclination here is to notice that if $\operatorname{Frac}\left(C^{\infty}([0,1], \mathbf{R}) / u_{\mathscr{F}}\right)$ is given the valuation

$$
v([f])=\min \left\{k \mid f^{(k)} \notin u_{\mathscr{F}}^{\#}\right\}
$$

we get an $\omega$-pseudo-complete Hensel field with cross-section, having as residue field $\operatorname{Frac}\left(C^{\infty}([0,1], \mathbf{R}) / u_{\mathscr{F}}^{\#}\right)$. There is one serious problem with this approach to proving Theorem 5.2.2: The valuation ideal is not contained in $C^{\infty}([0,1], \mathbf{R}) / w_{\mathscr{F}}$, as the following example shows.

EXAMPLE 5.2.3. Let $F=\{1 / n\} \cup\{0\}$ be in $\mathscr{F}$. Let

$$
f(x)=e^{-1 / x} \sin (\pi / x), \quad g(x)=e^{-1 /\left(x^{2}\right)} .
$$

Then $v([f])=1, v([g])=0$. Hence $v([f] /[g])=1$. However,

$$
[f] /[g]=\left[e^{\left(1 /\left(x^{2}\right)-1 / x\right)} \sin (\pi / x)\right] \notin C^{\infty}([0,1], \mathbf{R}) / q_{\mathscr{F}} .
$$

It might be objected that this example deals only with certain special $\mathscr{F}$. However, assuming Theorem 5.2.2 to be true, Theorem 5.2.2 and Example 5.2.3 show that the valuation ideal will not be contained in $C^{\infty}([0,1], \mathbf{R}) / q_{\mathscr{F}}$ for any $\mathscr{F}$ containing a rank one set $F$ with polynomial distances. In fact, for such an $\mathscr{F}$, this can be shown for any $w_{\mathscr{F}}$ as above. We cannot, therefore, easily use Ax-Kochen machinery to classify these domains. Instead, we shall use jets.

DeFINITION 5.2.4. For the purpose of this paper a jet is a sequence $\left\{f_{j}\right\}_{j=0}^{\infty}$ where $f_{j} \in C^{\infty}([0,1], \mathbf{R})$ for all $j \in \mathbf{N}$.

DEFINITION 5.2.5. A residue class jet $(\bmod p)$ is a sequence $\left\{\left[f_{j}\right]\right\}_{j=0}^{\infty}$ where $\left[f_{j}\right] \in C^{\infty}([0,1], \mathbf{R}) / p$.

Notation 5.2.6. $T$ denotes the set of all jets. $T / p$ denotes the set of all residue class jets $(\bmod p)$.

REMARK 5.2.7. $T$ and $T / p$ are commutative differential rings with

(i) elementwise addition,

(ii) multiplication defined: $\left\{f_{j}\right\}_{j=0}^{\infty} \cdot\left\{g_{j}\right\}_{j=0}^{\infty}=\left\{h_{j}\right\}_{j=0}^{\infty}$, where

$$
h_{j}=\sum_{k=0}^{j}\left(\begin{array}{l}
j \\
k
\end{array}\right) g_{k} \cdot f_{j-k},
$$

(iii) a derivation $D$ defined: $D\left(\left\{f_{j}\right\}_{j=0}^{\infty}\right)=\left\{f_{j+1}\right\}_{j=0}^{\infty}$. 
REMARK 5.2.8. We have a differential ring injection

$$
\theta_{u_{\mathscr{T}}}^{\#}: C^{\infty}([0,1], \mathbf{R}) / w_{\mathscr{F}} \rightarrow T /\left(u_{\mathscr{F}}^{\#}\right)
$$

defined

$$
\theta_{u_{\mathscr{F}}}^{\#}\left(\left[f+w_{\mathscr{F}}\right]\right)=\left\{\left[f^{(j)}+u_{\mathscr{F}}^{\#}\right]\right\}_{j=0}^{\infty} .
$$

The image of this map is a proper subring of $T /\left(u^{\#}+\mathscr{F}\right)$. Now let

$$
\psi: C^{\infty}([0,1], \mathbf{R}) / u_{\mathscr{F}}^{\#} \rightarrow C^{\infty}([0,1], \mathbf{R}) / u_{\mathscr{G}}^{\#}
$$

be an isomorphism. This clearly induces an isomorphism

$$
\Omega: T / u_{\mathscr{F}}^{\#} \rightarrow T / u_{\mathscr{G}}^{\#}
$$

defined by

$$
\Omega\left(\left\{\left[f_{j}\right]\right\}\right)=\left\{\psi\left(\left[f_{j}\right]\right)\right\} .
$$

As a candidate for $\xi$ in Theorem 5.2.2 we have

$$
\theta_{\left(u_{r}^{*}\right)}^{-1} \circ \Omega \circ \theta_{u_{F}^{\#}} \text {. }
$$

We need only show that

$$
\Omega\left(\theta_{u_{\mathscr{F}}^{*}}\left(C^{\infty}([0,1], \mathbf{R}) / u_{\mathscr{F}}^{\#}\right)\right)=\theta_{u_{\mathscr{G}}^{*}}\left(C^{\infty}([0,1], \mathbf{R}) / u_{\mathscr{G}}^{\#}\right)
$$

Proposition 5.2.9. For $\psi$ as in Theorem 5.2.2, $\Omega$ as above, and $\theta$ as in Remark 5.2.8, we have

$$
\Omega\left(\theta_{u_{\mathscr{F}}}^{\#}\left(t_{\mathscr{F}}^{\#} / w_{\mathscr{F}}\right)\right)=\theta_{u_{\mathscr{G}}}^{\#}\left(t_{\mathscr{G}}^{\#} / w_{\mathscr{G}}\right)
$$

Proof. If $f \in t_{\mathscr{F}}^{\#}$, then $f^{(j)} \in t_{\mathscr{F}}^{\#}$ for all $j \in \mathbf{N}$. Since by hypothesis, $\psi([g])=[h]$ implies $g^{(k)}(0)=h^{(k)}(0)$ for all $k$, we have that $f^{(j)} \in$ $t_{\mathscr{F}}^{\#}$ implies that there exists $h_{j} \in t_{\mathscr{G}}^{\#}$ such that $\psi\left(\left[f^{(j)}\right]\right)=\left[h_{j}\right]$. Finally Lemma 5.1 .10 gives the existence of $h \in t_{\mathscr{G}}^{\#}$ such that $h^{(j)} \equiv_{\mathscr{G}} h_{j}$. Hence

$$
\Omega\left(\theta_{u_{\mathscr{F}}^{*}}\left(\left[f+w_{\mathscr{F}}\right]\right)\right)=\theta_{u_{\mathscr{F}}^{*}}\left(\left[h+w_{\mathscr{G}}\right]\right) .
$$

This proves

$$
\Omega\left(\theta_{u_{\mathscr{F}}^{\#}}\left(t_{\mathscr{F}}^{\#} / w_{\mathscr{F}}\right)\right) \subset \theta_{u_{\mathscr{G}}^{*}}\left(t_{\mathscr{G}}^{\#} / w_{\mathscr{G}}\right) .
$$

The other inclusion follows by symmetry.

Proof of Theorem 5.2.2. (ii) $\rightarrow$ (iii). As we mentioned above we need only show that

$$
\Omega\left(\theta_{u_{\mathscr{T}}^{*}}\left(C^{\infty}([0,1], \mathbf{R}) / w_{\mathscr{F}}\right)\right)=\theta_{u_{\mathscr{\zeta}}^{*}}\left(C^{\infty}([0,1], \mathbf{R}) / w_{\mathscr{G}}\right) .
$$


In fact, we need only show that for $[f] \in C^{\infty}([0,1], \mathbf{R}) / w_{\mathscr{F}}$,

$$
\xi([f])=\theta_{\left(u_{\xi}^{*}\right)}^{-1} \circ \Omega \circ \theta_{u_{\mathscr{F}}^{*}}([f]) \in C^{\infty}([0,1], \mathbf{R}) / w_{\mathscr{G}} .
$$

So let $f, g \in C^{\infty}([0,1], \mathbf{R})$ be such that $\psi\left(\left[f+u_{\mathscr{F}}^{\#}\right]\right)=\left[g+u_{\mathscr{G}}^{\#}\right]$. Then by hypothesis, $f^{(k)}(0)=g^{(k)}(0)$ for all $k$. Hence, if $\psi\left(\left[f^{(k)}+u_{\mathscr{F}}^{\#}\right]\right)=\left[h_{k}+u_{\mathscr{G}}^{\#}\right]$, then $h_{k}^{(j)}(0)=f^{(k+j)}(0)=g^{(k+h)}(0)$ for all $j$ and so $\left(g^{(k)}-h_{k}\right) \in t_{\mathscr{G}}^{\#}$ for all $k$. Then, by Lemma 5.1.10, there exists $\zeta \in t_{\mathscr{G}}^{\#}$ such that $\left[\zeta^{(k)}+u_{\mathscr{G}}^{\#}\right]=\left[g^{(k)}-h_{k}+u_{\mathscr{G}}^{\#}\right]$ for all $k$. Then,

$$
\xi\left(\left[f+w_{\mathscr{F}}\right]\right)=\left[g-\zeta+w_{\mathscr{G}}\right] \in C^{\infty}([0,1], \mathbf{R}) / w_{\mathscr{G}} .
$$

(iii) $\rightarrow$ (ii). If $\xi\left(u_{\mathscr{F}}^{\#} / w_{\mathscr{F}}\right)=u_{\mathscr{G}}^{\#} / w_{\mathscr{G}}$, then this result follows immediately. In some cases, however, $\xi\left(u_{\mathscr{F}}^{\#} / w_{\mathscr{F}}\right) \neq u_{\mathscr{G}}^{\#} / w_{\mathscr{G}}$ (see Example 5.2.10). We need the following claim:

Claim 1. Let $f \in t_{\mathscr{F}}^{\#}$ be such that $f>_{\mathscr{F}} 0$ and such that $f^{(k)} \in u_{\mathscr{F}}^{\#}$ for all $k \geq 1$. Then for all $j \in \mathbf{N}$, there exists $h_{j} \in t_{\mathscr{F}}^{\#}$ such that $\left[h_{j}+w_{\mathscr{F}}\right]^{j}=\left[f+w_{\mathscr{F}}\right]$. That is, $\left[f+w_{\mathscr{F}}\right]$ has a $j$ th root.

Proof of Claim 1. Recall that $t_{\mathscr{F}}^{\#} / u_{\mathscr{F}}^{\#} \simeq t_{\mathscr{F}} / u_{\mathscr{F}}$ so all positive elements of $t_{\mathscr{F}}^{\#} / u_{\mathscr{F}}^{\#}$ have $j$ th roots in $t_{\mathscr{F}}^{\#} / u_{\mathscr{F}}^{\#}$ for all $j$. If $\left[g_{j}+u_{\mathscr{F}}^{\#}\right]^{j}=$ $\left[f+u_{\mathscr{F}}^{\#}\right]$, then $\left\{\left[g_{j}\right], 0,0, \ldots\right\}^{j}=\{[f], 0,0, \ldots\}$. By Lemma 5.1.10, there exists $h_{j} \in t_{\mathscr{F}}^{\#}$ such that $h_{j} \equiv \mathscr{F} g_{j}$ and $h_{j}^{(k)} \equiv_{\mathscr{F}} 0$ for all $k \geq 1$.

Claim 2. For $f, g \in t_{\mathscr{F}}^{\#}$, if $g^{(k)} \in u_{\mathscr{F}}^{\#}$ for all $k \geq 1$ and $|f|^{j}>_{\mathscr{F}}|g|$ for all $j$, then $\left[f+w_{\mathscr{F}}\right]$ divides $\left[g+w_{\mathscr{F}}\right]$ (in $\left.C^{\infty}([0,1], \mathbf{R}) / w_{\mathscr{F}}\right)$.

Proof of Claim 2. Quotient rule.

Observe now, that if $f \in u_{\mathscr{F}}^{\#}-w_{\mathscr{F}}$, then for some $j$, neither $\left[f+w_{\mathscr{F}}\right]$ nor $\left[-f+w_{\mathscr{F}}\right]$ will have a $j$ th root.

Claim 3. If $\xi\left(u_{\mathscr{F}}^{\#} / w_{\mathscr{F}}\right) \neq u_{\mathscr{G}}^{\#} / w_{\mathscr{G}}$, then there exist $g, h \in$ $C^{\infty}([0,1], \mathbf{R})$ such that

$$
\begin{aligned}
& u_{\mathscr{F}}^{\#}=\left\{\left.f|| f\right|_{\mathscr{F}}|h|^{j} \text { for all } j \in \mathbf{N}\right\}, \\
& u_{\mathscr{G}}^{\#}=\left\{\left.f|| f\right|_{\mathscr{G}}|g|^{j} \text { for all } j \in \mathbf{N}\right\} .
\end{aligned}
$$

Proof of Claim 3. Let $f \in u_{\mathscr{F}}^{\#}$ be such that $\xi\left(\left[f+w_{\mathscr{F}}\right]\right)=\left[g+w_{\mathscr{G}}\right]$, where $g \notin u_{\mathscr{G}}^{\#}$. Let $\rho \in t_{\mathscr{G}}^{\#}$ be such that $0<\mathscr{G} \rho<\mathscr{G}|g|^{j}$ for all 
$j \in \mathbf{N}$. Let $\zeta \in t_{\mathscr{G}}^{\#}$ be such that $\zeta \equiv_{\mathscr{G}} \rho$ and $\zeta^{(k)} \equiv_{\mathscr{G}} 0$ for all $k \geq 1$. Then $\left[g+w_{\mathscr{G}}\right]$ divides $\left[\zeta+w_{\mathscr{G}}\right]$. Hence $\left[\zeta+w_{\mathscr{G}}\right] \in \xi\left(u_{\mathscr{F}}^{\#} / w_{\mathscr{F}}\right)$. But $\left[\zeta+w_{\mathscr{G}}\right]$ has $j$ th roots for all $j \in \mathbf{N}$, so $\xi^{-1}\left(\left[\zeta+w_{\mathscr{G}}\right]\right)=\left[w_{\mathscr{F}}\right]$. Therefore $\left[\zeta+w_{\mathscr{G}}\right]=\left[w_{\mathscr{G}}\right], \zeta \in u_{\mathscr{G}}^{\#}$, and $\rho \in u_{\mathscr{G}}^{\#}$. Then

$$
\left\{\left.f|| f\left|<_{\mathscr{G}}\right| g\right|^{j} \text { for all } j \in \mathbf{N}\right\} \subset u_{\mathscr{G}}^{\#} \text {. }
$$

To see the other inclusion, recall that $u_{\mathscr{G}}^{\#}=u_{\mathscr{G}} \cap C^{\infty}([0,1], \mathbf{R})$ and is therefore convex. Hence if $f \in u_{\mathscr{G}}^{\#}$ is such that $|f|>_{\mathscr{G}}|g|^{j}$ for some $j$, then $g^{j} \in u_{\mathscr{G}}^{\#}$; hence $g \in u_{\mathscr{G}}^{\#}$, contrary to assumption. For the corresponding result for $u_{\mathscr{F}}^{\#}$, we use $\xi^{-1}$.

Therefore, to complete the proof of the theorem, we are left with the case

$$
\begin{aligned}
& u_{\mathscr{G}}^{\#}=\left\{\left.f|| f\right|_{\mathscr{F}}|h|^{j} \text { for all } j \in \mathbf{N}\right\}, \\
& u_{\mathscr{G}}^{\#}=\left\{\left.f|| f\right|_{\mathscr{G}}|g|^{j} \text { for all } j \in \mathbf{N}\right\} .
\end{aligned}
$$

We next denote

$$
\begin{aligned}
e_{\mathscr{G}}^{\#} & =\left\{\left.f|\exists j ;| f\right|^{j}<|h|\right\}, \\
e_{\mathscr{G}}^{\#} & =\left\{\left.f|\exists j ;| f\right|^{j}<_{\mathscr{G}}|g|\right\}
\end{aligned}
$$

where $h$ and $g$ are as in Claim 3. An Ax-Kochen argument yields the result if we can show that there exists an isomorphism

$$
\psi_{1}: C^{\infty}([0,1], \mathbf{R}) / e_{\mathscr{F}}^{\#} \rightarrow C^{\infty}([0,1], \mathbf{R}) / e_{\mathscr{G}}^{\#}
$$

such that $\psi_{1}\left(\left[\zeta+e_{F}^{\#}\right]\right)=\left[\rho+e_{\mathscr{G}}^{\#}\right]$ implies $\zeta^{(k)}(0)=\rho^{(k)}(0)$ for all $k \in \mathbf{N}$. This, in turn, will follow automatically if we show that $\xi\left(e_{\mathscr{F}}^{\#} / w_{\mathscr{F}}\right)=e_{\mathscr{G}}^{\#} / w_{\mathscr{G}}$. So let

$$
\begin{array}{r}
A_{\mathscr{F}}=\left\{\gamma \in t_{\mathscr{F}}^{\#} \mid \exists j \in \mathbf{N}, \exists \zeta, \rho \in t_{\mathscr{F}}^{\#} ; \gamma^{j}=\zeta+\rho,\left[\zeta+w_{\mathscr{F}}\right] \in\left(u_{\mathscr{F}}^{\#} / w_{\mathscr{F}}\right),\right. \\
\text { and } \left.\left[\rho+w_{\mathscr{F}}\right] \in \xi^{-1}\left(u_{\mathscr{G}}^{\#} / w_{\mathscr{G}}\right)\right\} . \\
A_{\mathscr{G}}=\left\{\gamma \in t_{\mathscr{G}}^{\#} \mid \exists j \in \mathbf{N}, \exists \zeta, \rho \in t_{\mathscr{G}}^{\#} ; \gamma^{j}=\zeta+\rho,\left[\zeta+w_{\mathscr{G}}\right] \in\left(u_{\mathscr{G}}^{\#} / w_{\mathscr{G}}\right),\right. \\
\text { and } \left.\left[\rho+w_{\mathscr{G}}\right] \in \xi\left(u_{\mathscr{F}}^{\#} / w_{\mathscr{F}}\right)\right\} .
\end{array}
$$

Clearly $\xi\left(A_{\mathscr{F}} / w_{\mathscr{F}}\right)=A_{\mathscr{G}} / w_{\mathscr{G}}$. So, if we can show that $A_{\mathscr{F}}=e_{\mathscr{F}}^{\#}$, the theorem will be proved. By the proof of Claim 3, $A_{\mathscr{F}} \subset e_{\mathscr{F}}^{\#}$ and $A_{\mathscr{G}} \subset e_{\mathscr{G}}^{\#}$. We need to show that $e_{\mathscr{F}}^{\#} \subset A_{\mathscr{F}}$ and $e_{\mathscr{G}}^{\#} \subset A_{\mathscr{G}}$. Let

$$
\left[\rho+w_{\mathscr{F}}\right] \in \xi^{-1}\left(u_{\mathscr{G}}^{\#} / w_{\mathscr{G}}\right), \quad\left[\rho+w_{\mathscr{F}}\right] \notin\left(u_{\mathscr{F}}^{\#} / w_{\mathscr{F}}\right) .
$$


There exists $\zeta \in u_{\mathscr{F}}^{\#}$ such that $\zeta^{(k)} \equiv \rho^{(k)}$ for all $k \geq 1$. So $(\zeta-\rho) \in A_{\mathscr{F}}$ and $(\zeta-\rho)^{(k)} \equiv_{\mathscr{F}} 0$ for all $k \geq 1$. Since $(\zeta-\rho) \notin u_{\mathscr{F}}^{\#}$, there exists $j$ such that $|\zeta-\rho|>_{\mathscr{F}}|h|^{j}$.

Next, let $\sigma \in e_{\mathscr{F}}^{\#}$ be such that $\sigma^{(k)} \in u_{\mathscr{F}}^{\#}$ for all $k \geq 1$. Since for some $m \in \mathbf{N},|\zeta-\rho|>_{\mathscr{F}}|h|^{j}>_{\mathscr{F}}|\sigma|^{j m}$, we have that $\{[\zeta-\rho]$, $0,0, \ldots\}$ divides $\left\{\left[\sigma^{j m+1}\right], 0,0, \ldots\right\}$. Therefore $\sigma \in A_{\mathscr{F}}$.

Next, let $\beta \in e_{\mathscr{g}}^{\#}$. There exists $\zeta_{2} \in u_{\mathscr{F}}^{\#}$ such that $\beta^{(k)} \equiv_{\mathscr{F}} \zeta_{2}^{(k)}$ for all $k \geq 1$. Thus $\left(\beta-\zeta_{2}\right)$ has the conditions of $\sigma$ above, and hence $\left(\beta-\zeta_{2}\right) \in A_{\mathscr{F}}$ and $\beta \in A_{\mathscr{F}}$. By symmetry $e_{\mathscr{G}}^{\#} \subset A_{\mathscr{G}}$. This proves (iii) $\rightarrow$ (ii) and completes the proof of Theorem 5.2.2.

We mentioned that $\xi\left(u_{\mathscr{F}}^{\#} / w_{\mathscr{F}}\right)$ need not equal $u_{\mathscr{G}}^{\#} / w_{\mathscr{G}}$. The following is an example.

EXAMPLE 5.2.10. Let $h(x)=x-e^{-1 / x}$. Let $F=\{1 / n\}_{n=1}^{\infty} \cup\{0\}$ be in nonmaximal $\mathscr{F}$. Define $\mathscr{G}$,

$$
\mathscr{G}=\{G \mid h(G) \in \mathscr{F}\} .
$$

Define $u_{\mathscr{G}}^{\#}$ and $u_{\mathscr{F}}^{\#}$

$$
\begin{aligned}
& u_{\mathscr{G}}^{\#}=\left\{f\left|e^{-k / x}>_{\mathscr{F}}\right| f \mid \text { for all } k \in \mathbf{N}\right\}, \\
& u_{\mathscr{G}}^{\ddot{*}}=\left\{f\left|e^{-k / x}>_{\mathscr{G}}\right| f \mid \text { for all } k \in \mathbf{N}\right\} .
\end{aligned}
$$

It can be shown, by Taylor's Theorem, that $w_{\mathscr{F}}=w_{\mathscr{G}}$. But $u_{\mathscr{F}}^{\#} \neq u_{\mathscr{G}}^{\#}$ since $e^{-1 / x} \sin (\pi / x) \in u_{\mathscr{F}}^{\#}-u_{\mathscr{G}}^{\#}$. Hence, we have

$$
\text { id: } C^{\infty}([0,1], \mathbf{R}) / w_{\mathscr{F}} \rightarrow C^{\infty}([0,1], \mathbf{R}) / w_{\mathscr{G}}
$$

and

$$
\operatorname{id}\left(u_{\mathscr{F}}^{\#} / w_{\mathscr{F}}\right)=u_{\mathscr{F}}^{\#} / w_{\mathscr{F}} \neq u_{\mathscr{G}}^{\#} / w_{\mathscr{G}} .
$$

The diffeomorphism $h$, of course, does induce an isomorphism such that $\xi\left(u_{\mathscr{F}}^{\#} / w_{\mathscr{F}}\right)=u_{\mathscr{G}}^{\#} / w_{\mathscr{G}}$. Surprisingly, it induces more than one such isomorphism. We define $\xi_{1}$ and $\xi_{2}$

$$
\begin{aligned}
& \xi_{1}\left(\left[f+w_{\mathscr{F}}\right]\right)=\left[f \circ h+w_{\mathscr{\xi}}\right], \\
& \xi_{2}\left(\left\{[f],\left[f^{\prime}\right],\left[f^{\prime \prime}\right], \ldots\right\}\right)=\left\{[f \circ h],\left[f^{\prime} \circ h\right], \ldots\right\} .
\end{aligned}
$$

We also have a surprising corollary to Theorem 5.2.2.

Corollary 5.2.11. Let $\mathscr{F}$ be as in Theorem 5.2.2. Let

$$
q_{\mathscr{F}}=\left\{f \mid Z\left(f^{(n)}\right) \in \mathscr{F} \text { for all } n \geq 0\right\} .
$$


Then there exists a prime ideal $w_{\mathscr{F}}, q_{\mathscr{F}} \subsetneq w_{\mathscr{F}}$, and an isomorphism $\xi$,

$$
\xi: C^{\infty}([0,1], \mathbf{R}) / q_{\mathscr{F}} \rightarrow C^{\infty}([0,1], \mathbf{R}) / w_{\mathscr{F}}
$$

such that

$$
\xi(D[f])=D^{\prime}(\xi([f]))
$$

Proof. Let

$$
u_{\mathscr{F}}=\left\{f|| f \mid<e^{-1 /(k x)} \text { for some } k\right\} .
$$

Then $C([0,1], \mathbf{R}) / p_{\mathscr{F}} \simeq C([0,1], \mathbf{R}) / u_{\mathscr{F}}$ and the result follows by Theorem 5.2.2.

Combining Theorem 5.2.2 with 3.2.26 we see that we have classified 9 domains $C^{\infty}([0,1], \mathbf{R}) / u_{\mathscr{F}}^{\#}$ and 9 domains $C^{\infty}([0,1], \mathbf{R}) / w_{\mathscr{F}}$. We also have $C^{\infty}([0,1], \mathbf{R}) / Q \simeq \mathbf{R}[[x]]$ and $C^{\infty}([0,1], R) / \mathbf{m} \simeq$ $\mathbf{R}$. Altogether, we have classified 20 residue class domains of $C^{\infty}([0,1], \mathbf{R})$.

We shall show now that there exist prime ideals $p, q_{\mathscr{F}} \subset p, \mathscr{F}$ as in Theorem 5.2.2, such that $C^{\infty}([0,1], \mathbf{R}) / p$ is not one of the domains we have classified.

REMARK 5.2.12. For all the domains we have so far classified, there is no $\sqrt{-1}$ in the fraction field.

LEMMA 5.2.13. Let $R$ be any commutative ring. For $f, g \in R$, if $f$ is in every prime ideal that contains $g$, then for some $k \in \mathbf{N}, g$ divides $f^{k}$.

Proof. Assume that for all $k \in \mathbf{N}, g$ does not divide $f^{k}$. Then letting $I=\{g h \mid h \in R\}$, we have that $I \cap\left\{f, f^{2}, \ldots, f^{n}, \ldots\right\}=\varnothing$. By Zorn's Lemma there exists an ideal $J$, such that

(a) $I \subset J$,

(b) $J \cap\left\{f, f^{2}, \ldots, f^{n}, \ldots\right\}=\varnothing$, and

(c) $J$ is maximal with respect to (b).

To see that $J$ is prime, let $h k \in J$ and assume toward a contradiction that $f^{n}=j_{1}+k x$ and $f^{m}=j_{2}+h y$ (with $j_{1}, j_{2} \in J$ ). But then

$$
f^{m+n}=\left(j_{1} j_{2}+j_{2} k x+j_{1} h y+k h x y\right) \in J .
$$


TheOREM 5.2.14. Let $\mathscr{F}$ be as in Theorem 5.2.2. Let $q_{\mathscr{F}}$ be as in Notation 5.1.1. Then there exists a prime ideal $p \subset C^{\infty}([0,1], \mathbf{R})$, such that $q_{\mathscr{F}} \subset p$ and $\sqrt{-1} \in \operatorname{Frac}\left(C^{\infty}([0,1], \mathbf{R}) / p\right.$.

Proof. Let $\left[\{1 / n\}_{n=1}^{\infty} \cup\{0\}\right] \in \mathscr{F}$. As we mentioned earlier, this assumption involves no loss of generality. Define $f$ and $g$ in $C^{\infty}([0,1], \mathbf{R})$ by

$$
\begin{aligned}
& f(x)=e^{-1 / x} \sin (\pi / x), \\
& g(x)=e^{-\left(1 /\left(x^{2}\right)\right)} .
\end{aligned}
$$

It can be shown that $f^{2}+g^{2}$ does not divide $g^{k}\left(\bmod q_{\mathscr{F}}\right)$ for any $k \in \mathbf{N}$. That is, (since $\sin (n \pi)=0$ ), we can show that for any $k$, $\pm \infty$ are the only possible limit points of

$$
\left\{\left(\frac{g^{k}}{f^{2}+g^{2}}\right)^{(k+1)}\left(\frac{1}{n}\right)\right\}_{n=1}^{\infty} .
$$

So by Lemma 5.2.13, there exists a prime ideal $p_{1} \subset C^{\infty}([0,1], \mathbf{R}) / q_{\mathscr{F}}$ such that $\left[f^{2}+g^{2}+q_{\mathscr{F}}\right] \in p_{1}$ and $\left[g+p_{\mathscr{F}}\right] \notin p_{1}$. Hence, there exists a prime ideal $p \subset C^{\infty}([0,1], \mathbf{R})$ such that $q_{\mathscr{F}} \subset p,\left(f^{2}+g^{2}\right) \in p$, and $g \notin p$. Therefore, in $C^{\infty}([0,1], \mathbf{R}) / p,[f]^{2}+[g]^{2}=0,[f] \neq 0$, $[g] \neq 0$; and in the fraction field

$$
\frac{[f]}{[g]}=\sqrt{-1} .
$$

REMARK 5.2.15. This also shows the existence of a prime ideal $p \subset$ $C^{\infty}([0,1], \mathbf{C})$ such that $p$ is not equal to its own complex conjugate.

\section{REFERENCES}

[1] J. Ax and S. Kochen, Diophantine problems over local fields, II, Amer. J. Math., 87 (65), 631-648.

[2] D. Booth, Ph.D. Thesis, Univ. of Wisconsin, Madison, Wisc. 1969.

[3] W. Baur, On the elementary theory of pairs of real closed fields, II, J. Symbolic Logic, 47 (1982), 669-679.

[4] C. C. Chang and H. J. Keisler, Model Theory, North Holland Publishing Co., Amsterdam, 1973.

[5] G. Cherlin, Rings of continuous functions: Decision problems, Model Theory of Algebra and Arithmetic 44-91, Lecture Notes in Math. 834, Springer, Berlin, 1980.

[6] G. Cherlin and M. Dickmann, Anneaux reél clos et anneaux des fonctions continues, C. R. Acad. Sci. Paris Ser. A-B, 290 (1980), no. 1, A1-A4.

[7] _ Real Closed Rings II, Model Theory, Ann. Pure and Applied Logic, 25 (1983), 213-231. 
[8] — Real Closed Rings I, Residue rings of rings of continuous functions, Fund. Math., 126 (1986), 147-183.

[9] W. W. Comfort and S. Negrepontis, The Theory of Ultrafilters, Springer, New York, 1974.

[10] L. Gillman and M. Jerison, Rings of Continuous Functions, D. Van Nostrand Co., Princeton, N.J., 1960.

[11] M. Hendriksen and D. G. Johnson, On the structure of a class of Archimedean lattice-ordered algebras, Fund. Math., 50 (1961), 73-94.

[12] M. Henriksen, J. Isbell and D. G. Johnson, Residue class fields of lattice-ordered algebras, Fund. Math., 50 (1961), 107-117.

[13] N. Jacobson, Basic Algebra, II, W. H. Freeman and Co., San Francisco, 1980.

[14] S. Kochen, The Model Theory of Local Fields, Logic Conference Kiel 1974, 384-421, Lecture Notes in Math. 499, Springer, Berlin, 1975.

[15] C. W. Kohls, Ideals in rings of continuous functions, Fund. Math., 45 (1957), 28-50.

[16] _ Prime ideals in rings of continuous functions, Illinois J. Math., 2 (1958), 505-536.

[17] __ Prime ideals in rings of continuous functions II, Duke Math. J., 25 (1958), 447-458.

[18] _ Prime z-filters on completely regular spaces, Trans. Amer. Math. Soc., 120 (1965), 236-246.

[19] J. Moloney, First Order Theories of Residue Domains of Rings of Continuous Functions, Thesis, Rutgers University (1986).

[20] J. R. Munkres, Topology, a First Course, Prentice Hall, Inc. Englewood Cliffs, N.J., 1975.

[21] W. Rudin, Homogeneity problems in the theory of Cech compactifications, Duke Math. J., 23 (1956), 409-416.

[22] A. Tarski, A Decision Model for Elementary Algebra and Geometry, University of California Press, Berkeley, 1951.

[23] J. Van Mill, An Introduction to $\beta \omega$, Handbook of Set Theoretic Topology, 503-567, North-Holland, Amsterdam, 1984.

[24] E. Wimmers, The Shelah P-point Independence Theorem, Israel J. Math., 43 (1982), 28-48.

Received October 28, 1986 and in revised form March 10, 1988.

MARShall UNIVERSITY

HUNTINGTON, WV 25701 



\section{PACIFIC JOURNAL OF MATHEMATICS EDITORS}

\author{
V. S. VARADARAJAN \\ (Managing Editor) \\ University of California \\ Los Angeles, CA 90024-1555-05 \\ Herbert Clemens \\ University of Utah \\ Salt Lake City, UT 84112 \\ Thomas ENRIGHT \\ University of California, San Diego \\ La Jolla, CA 92093
}

R. FINN

Stanford University

Stanford, CA 94305

Hermann FlaschKa

University of Arizona

Tucson, AZ 85721

VAughan F. R. Jones

University of California

Berkeley, CA 94720

Steven Kerckhoff

Stanford University

Stanford, CA 94305
RobION KIRBY

University of California

Berkeley, CA 94720

C. C. MOore

University of California

Berkeley, CA 94720

HAROLd STARK

University of California, San Diego La Jolla, CA 92093

\begin{tabular}{|c|c|c|c|c|}
\hline & ASSOCIA & E EDI & & \\
\hline R. ARENS & $\begin{array}{l}\text { E. F. BECKENBACH } \\
(1906-1982)\end{array}$ & NeumanN & $\begin{array}{c}\text { F. WoLF } \\
(1904-1989)\end{array}$ & K. Yoshida \\
\hline & SUPPORTINC & INSTI & TIONS & \\
\hline UNIVERSITY & ARIZONA & UNIVERS & OF OREGON & \\
\hline UNIVERSITY & BRITISH COLUMBIA & UNIVER & OF SOUTHEI & CALIFORNIA \\
\hline CALIFORNIA & STITUTE OF TECHNOLOGY & STANFOI & UNIVERSITY & \\
\hline UNIVERSITY & CALIFORNIA & UNIVER & OF HAWAII & \\
\hline MONTANA S & TE UNIVERSITY & UNIVER & OF TOKYO & \\
\hline UNIVERSITY & NEVADA, RENO & UNIVER & OF UTAH & \\
\hline NEW MEXIC & TATE UNIVERSITY & WA & N STATE UN & ERSITY \\
\hline OREGON ST & UNIVERSITY & & OF WASHINC & $\mathrm{ON}$ \\
\hline
\end{tabular}




\section{Pacific Journal of Mathematics}

\section{Vol. 143, No. $1 \quad$ March, 1990}

Walter Bergweiler, On the fix-points of composite functions $\ldots \ldots \ldots \ldots \ldots 1$ Aldo Biancofiore, Maria Lucia Fania and Antonio Lanteri, Polarized surfaces with hyperelliptic sections $\ldots \ldots \ldots \ldots \ldots \ldots \ldots \ldots \ldots$

Ciprian Borcea, Deforming varieties of $k$-planes of projective complete

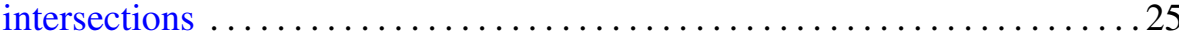

Morton Brown, Fixed points for orientation preserving homeomorphisms of

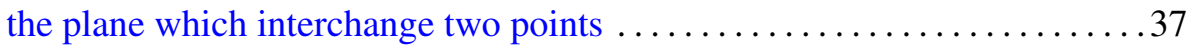

Hao Zhi Chuan, Note on the inequality of the arithmetic and geometric means

Paul Henry Edelman and Dennis E. White, Codes, transforms and the

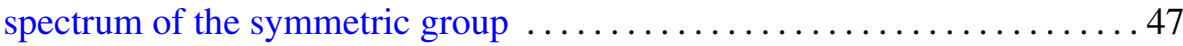

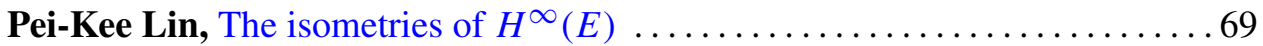

James J. Moloney, Residue class domains of the ring of convergent

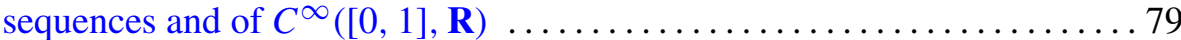

Zhong-Jin Ruan, The structure of pure completely bounded and completely

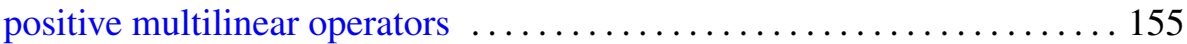

Wolfgang Ruess and William H. Summers, Weakly almost periodic semigroups of operators

Gideon Schwarz, A pretender to the title "canonical Moebius strip" . . . . . . 195

Ryszard Szwarc, Banach algebras associated with spherical representations of the free group 